\title{
La Silla QUEST RR Lyrae Star Survey: Region I
}

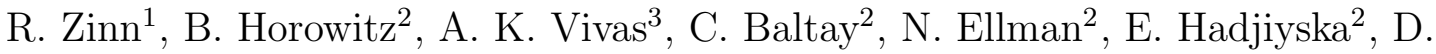 \\ Rabinowitz ${ }^{2}$, and L. Miller ${ }^{1}$
}

Received __; accepted _

accepted by ApJ

\footnotetext{
${ }^{1}$ Department of Astronomy, Yale University, P.O. Box 208101, New Haven, CT 065208101

${ }^{2}$ Department of Physics, Yale University, P.O. Box 208120, New Haven, CT 06511-8499

${ }^{3}$ Centro de Investigaciones de Astronomía, Apartado Postal 264, Mérida 5101-A, Venezuela
} 


\begin{abstract}
A search for RR Lyrae stars (RRLS) in $\sim 840 \mathrm{deg}^{2}$ of the sky in right ascension $150^{\circ}-210^{\circ}$ and declination $-10^{\circ}-+10^{\circ}$ yielded 1013 type ab and 359 type $\mathrm{c}$ RRLS. This sample is used to study the density profile of the Galactic halo, halo substructures, and the Oosterhoff type of the halo over distances $\left(d_{\odot}\right)$ from $\sim 5$ to $\sim 80 \mathrm{kpc}$. The halo is flattened towards the Galactic plane, and its density profile steepens in slope at galactocentric distances greater than $\sim 25 \mathrm{kpc}$. The RRLS in the stellar stream from the Sagittarius dwarf spheroidal (dSph) galaxy match well the model of Law \& Majewski for the stars that were stripped 1.3 to 3.2 Gyr ago, but not for the ones stripped 3.2 to 5.0 Gyr ago. Over densities are found at the locations of the Virgo Overdensity and the Virgo Stellar Stream. Within $1^{\circ}$ of $1220-1$, which Jerjen et al. identify as a halo substructure at $d_{\odot} \sim 24$ kpc, there are 4 RRLS that are possibly members. Away from substructures, the RRLS are a mixture of Oosterhoff types I and II, but mostly OoI ( 73\%). The accretion of galaxies resembling in RRLS content the most massive Milky Way satellites (LMC, SMC, For, Sgr) may explain this preponderance of OoI. Six new RRLS and 3 new anomalous Cepheids were found in the Sextans dSph galaxy.
\end{abstract}

Subject headings: Galaxy: halo — stars: variables: RR Lyrae 


\section{Introduction}

The properties of the Galactic halo and how they may be explained in the broader context of galaxy evolution have been a major area of research for more than 50 years. Early investigations of the halo, which by today's standards relied on tiny samples of halo stars and globular clusters, concluded that the halo is very old and uniform in age and has smooth contours of stellar density. The evolutionary picture that emerged from these studies, painted in the seminal paper by Eggen et al. (1962) (ELS), was that the halo formed rapidly during an early stage of the collapse of a single large gas cloud that later became the galactic disk. In today's terms, the ELS picture is an example of in situ formation, because it envisioned that the formation of stars and globular clusters occurred within the confines of the Milky Way (MW).

Later it was realized that in situ formation was probably not the origin of the whole halo, in particular its outer parts, because the ELS picture failed to explain the distribution and gradients in metallicity and in horizontal-branch (HB) morphology of the halo globular clusters (GCs) (Searle \& Zinn 1978) and the correlations between metallicity and kinematics of local samples of metal-poor stars (Norris et al. 1985; Carney et al. 1990). This motivated an accretion picture in which some halo stars and GCs formed in separate, small galaxies that subsequently disintegrated as they merged with the MW, depositing their already formed stars and star clusters into the halo. This picture has received very strong observational support in recent years by the discovery of the Sagittarius dwarf spheroidal galaxy (dSph), which is currently undergoing tidal disruption, the discoveries of several stellar streams and overdensities in the halo that are probably the remains of accreted dwarf galaxies or GCs (e.g., Helmi et al. 1999; Ibata et al. 2001b; Vivas et al. 2001; Newberg et al. 2002; Grillmair 2006; Duffau et al. 2006; Clewlev \& Kinman 2006;

Belokurov et al. 2007a.,b; Jurić et al. 2008; Schlaufman et al. 2009; Morrison et al. 2009; 
Bonaca et al. 2012; Sesar et al. 2012; Drake et al. 2013b), and the discoveries of many "ultra-faint" dwarf galaxies (UFD) in the halo (e.g., Willman et al. 2005; Zucker et al. 2006; Belokurov et al. 2006), which along with the previously known dSph galaxies are probably the survivors of a larger population of similar objects that have already merged with the MW. The existence of large stellar streams in the halos of other galaxies (e.g., Shang et al. 1998; Ibata et al. 2001a; Zucker et al. 2004; Martínez-Delgado et al. 2009) most notably in M31 where some appear to contain globular clusters as well as stars (Mackey et al. 2010), provide conclusive evidence that accretion is a widespread phenomenon.

While over the past 30 years many investigators have speculated that the halo may be the result of both in-situ formation and accretion, several recent studies have greatly strengthend the evidence. For example, from a study of the kinematics and metallicities of thousands of stars near the main-sequence turnoff, Carollo et al. (2007, 2010) concluded that the halo consists to two over-lapping components. The inner halo, galactocentric distances, $R_{g c}$, less than $10-15 \mathrm{kpc}$, is dominated by a relatively metal rich component $(\langle[\mathrm{Fe} / \mathrm{H}]\rangle=-1.6)$ that is flattened towards the galactic plane and has zero or slightly prograde rotation. In contrast, the outer halo, which dominates at $R_{g c} \gtrsim 15 \mathrm{kpc}$, is nearly round, in retrograde rotation, and significantly more metal-poor $(\langle[F e / H]\rangle=-2.2)$. This picture, which has spurred controversy (see Schönrich et al. 2011; Beers et al. 2012), has been supported by recent investigations of blue horizontal branch (BHB) and RR Lyrae stars (RRLS)(e.g., Kinman et al. 2012; Hattori et al. 2013). This dual halo may be explained by in-situ formation and accretion dominating, respectively, the inner and outer halos (Carollo et al. 2007, 2010).

Important age information on the two halos is provided by the GC systems. When the halo GCs are divided into young halo $(\mathrm{YH})$ and old halo $(\mathrm{OH})$ groups on the basis of the variation of $\mathrm{HB}$ morphology at constant $[\mathrm{Fe} / \mathrm{H}]$ (i.e., the $2^{\text {nd }}$ parameter effect), which is a 
rough proxy for cluster age (e.g., Dotter et al. 2011), they are also divided by kinematics and by spatial distribution (Zinn 1993; Da Costa \& Armandroff 1995); Mackey \& Gilmore 2004; Lee et al. 2007). The very old ages, mean prograde rotation and concentration at small $R_{g c}$ of the $\mathrm{OH}$ group suggest that the majority of them formed in situ, probably during an early stage of the dissipational collapse that led to the formation of the bulge and disk and the concomitant bulge/disk population of GCs. In contrast, the much more widely distributed and kinematically hotter $\mathrm{YH}$ clusters, and in addition a small fraction of the $\mathrm{OH}$ clusters that resemble $\mathrm{YH}$ clusters in these properties, are probably debris from accretion events. This is certainly the case for the clusters that can be unambiguously identified with the stellar streams from the Sgr dSph galaxy (Carraro et al. 2007; Law \& Majewski 2010a). The recent discovery that the $\mathrm{YH}$ clusters have a planar spatial distribution that is very similar to the one of the dSph and UDF galaxies indicates a common origin and is additional evidence that these clusters originated in now destroyed satellite galaxies (Keller et al. 2012; Pawlowski et al. 2012). The wide range in age of the YH clusters $(\sim 5$ Gyrs Dotter et al. 2011; Carraro et al. 2007) suggests that a fraction of the accreted halo originated in several slowly evolving dwarf galaxies that formed globular clusters over extended periods of time, not unlike the Sgr and Fornax dSph galaxies (Zinn 1993; Da Costa \& Armandroff 1995; Mackey \& Gilmore 2004; Forbes \& Bridges 2010). However, the low mean [Fe/H] of the outer halo suggests that much of it originated in low-mass systems that never formed clusters and experienced less chemical evolution (Carollo et al. 2007, 2010).

The first investigations of the relative abundances of the elements in the dSph galaxies indicated that they do not resemble the metal-poor stars near the Sun in the ratio of the $\alpha$ elements to Fe (e.g., Shetrone et al. 2001), which cast doubt on the hypothesis that satellite accretion played a role in halo formation. As larger samples stars in the galaxies were measured, it became clear that the very metal-poor stars in the galaxies and in the halo had similar abundance ratios, but not the more metal-rich ones. Since many of the 
surviving dSph galaxies have experienced long histories of star formation and chemical enrichment, it was argued that the the abundances of their metal-rich stars may not be indicative of the stars in dwarf galaxies that were accreted in the early evolution of the MW (see reviews by Geisler et al. 2007; Tolstoy et al. 2009). Most of the halo stars that were used in these comparisons with the dSph galaxies are members of the inner halo. Some recent investigations of the chemical abundances of halo stars have separated them into inner and outer halo samples on the basis of kinematics, which has led to the discovery of important differences. Roederen (2009) found that the inner halo is chemically more homogeneous than the outer halo, which can be explained if the accretion of dwarf galaxies with different chemical enrichment histories played a larger role in the outer than in the inner halo. A series of recent papers (Nissen \& Schuster 2010, 2011; Schuster et al. 2012) has shown that there are correlations between $\alpha / \mathrm{Fe}$ and other abundance ratios and the kinematics and the ages of halo stars. The stars in the outer halo are 2-3 Gyrs younger and have lower $\alpha / F e$ than the ones in the inner halo, again consistent with more accretion in the outer halo. There is evidence that satellite accretion also contributed to the inner halo, for Sheffield et al. (2012) have shown that some relatively nearby halo M giants have abundance ratios that are unlike the majority of halo stars, but similar to the stars in dwarf galaxies. In a reversal of what was once the case, the comparisons of the chemical compositions of the satellite dwarf galaxies and halo stars provide strong evidence that the accretion of similar galaxies contributed to the MW halo (see also Frebel \& Norris 2013).

Clearly, great progress has been made in understanding the formation of the halo, but there is a need for more observations. The number and the characteristics of the accretion events and of the surviving satellite galaxies provide tests of the relative importance of the accretion and in situ scenarios. While there is universal agreement that halo substructures exist, estimates of the fraction of the halo contained in substructures vary substantially (e.g., Bell et al. 2008; Xue et al. 2011; Deason et al. 2011). The transition zone from 
$R_{g c} \sim 10$ to $\sim 30 \mathrm{kpc}$ is of particular interest, because it is not clear if the properties of the halo vary smoothly or if there is a fairly abrupt transition. Because the MW is the best observed galaxy, the observations of its halo provide important constraints on simulations of galaxy formation in a cosmological context (e.g., Bullock \& Johnston 2005; Zolotov et al. 2009; Font et al. 2011; Helmi et al. 2011). With the goals providing additional data on halo structure and the presence and absence of stellar streams over the range of roughly 10 to $80 \mathrm{kpc}$, we have embarked on this series of papers.

This paper reports the first results of the La Silla - QUEST (LSQ) survey for RR Lyrae stars (RRLS) which has employed the 1-m Schmidt telescope at La Silla, in Chile and the QUEST camera. RRLS are, of course, pulsating horizontal branch stars with very characteristic periods ( 0.2 to 0.9 day) and lightcurves. Because their amplitudes vary between about 0.2 and 1.3 magnitudes in $\mathrm{V}$, they are easily detected in multi-epoch photometric surveys. The most important property of RRLS as probes of the halo is that they are excellent standard candles and, as previous surveys have shown (e.g., Ivezić et al. 2000; Vivas et al. 2001; Keller et al. 2008; Watkins et al. 2009; Sesar et al. 2010; Drake et al. 2013b), can be used to detect halo substructure.

In this paper, we describe our methods for selecting candidate RRLS and measuring the periods and lightcurves for the RRLS that we have identified in contiguous region of $\sim 840 \mathrm{deg}^{2}$, spanning the constellations Sextans to Virgo. We use these stars to investigate the density profile the Galactic Halo and find a significant break at $R_{g c} \sim 25 \mathrm{kpc}$. We also investigate the previously identified overdensities in the region, the leading stream from the Sgr dSph galaxy and the Virgo Stellar Stream, and examine the data for signatures of other streams. Finally, we investigate the pulsational properties of the RRLS (i.e., the Oosterhoff Effect) and compare the halo RRLS to those found in the four most massive satellite galaxies of the MW. We also see if there are variations in RRLS properties across 
the break in the density profile of the halo.

\section{Observations}

\subsection{Instrumentation}

The RRLS survey reported here uses the data from the La Silla-QUEST Southern Hemisphere Variability Survey (Hadjiyska et al. 2012), which is primarily a survey for

supernovae (Baltay et al. 2013) and trans-Neptunian objects. The Survey employs the 10 square degree QUEST Camera (Baltay et al 2007) at the prime focus of the 1 meter ESO Schmidt Telescope at the La Silla Observatory in Chile. The survey commenced observations in September of 2009 and uses $90 \%$ of the telescope time. For both the La Silla-QUEST (LSQ) RRLS and supernovae surveys, the telescope was used in the point-and-stare mode with 60s exposures, rather than the drift-scan mode, which was used in the Venezuelan QUEST RRLS survey (Vivas et al. 2004) (hereafter, simply the QUEST survey). Typically, each night of observation consisted of two visits to the same position, separated by $\sim 2$ hours, which allowed the elimination of moving objects from the data. Within one or two days, the observations were repeated, and the time spans over which observations at same position were obtained ranged from $\sim 25$ to $\sim 90$ days.

The camera consists of 112 CCD detectors arranged in a 4 by 28 array ( 4 rows, labeled A, B, C, and D, with 28 CCD's each) as shown in Figure 1, The progression of the 28 CCDs on a row are in the declination direction, with CCD 1 on the north side and CCD 28 on the south side of the field of view. Finger $\mathrm{A}$ is on the east side and finger $\mathrm{D}$ is on the west side of the field.

The CCD's are 600 x 2400 pixel thinned, back illuminated SARNOFF devices with $13 \mu \mathrm{m} \times 13 \mu \mathrm{m}$ pixels and a plate scale of $0.86 \operatorname{arcsec} /$ pixel. The camera covers $3.6^{\circ} \times 4.6^{\circ}$ 
on the sky with an active area of $9.6 \mathrm{deg}^{2}$, when all 112 CCDs are operating. During the RRLS survey, $\sim 100$ CCDs, $89 \%$ of the active area was utilized.

The survey uses a very broad-band filter to enable the detection of faint sources in short exposures. Plotted in Figure 2 is the normalized response function $(S(\lambda))$ of this filter and the camera CCD's, which was determined from the transmission curve of the filter and the spectral sensitivity of a typical CCD (Baltay et al. 2007). Because the latter is based on measurements for $\lambda \leq 6000 \AA$ and only estimated at longer wavelengths, the $S(\lambda)$ in Figure 2 should be considered approximate. The sharp decline at $\lambda \sim 7000 \AA$ has the desirable property of eliminating the fringing that plagues observations that are obtained at longer wavelengths. Defining the effective wavelength as $\lambda_{\text {eff }}=\int_{0}^{\infty} \lambda S(\lambda) \mathrm{d} \lambda$, we obtain $\lambda_{e f f}=5690 \AA$ and $2880 \AA$ for the full-width at half maximum (FWHM). Also plotted in Figure 2 is the $S(\lambda)$ of the Johnson V pass-band (Bessell 1990), from which we obtain, for comparison, $\lambda_{e f f}=5510 \AA$ and FWHM $=850 \AA$. Although the $S(\lambda)$ of the LSQ survey is offset in $\lambda_{\text {eff }}$ and is considerably broader than that of the standard $\mathrm{V}$ pass-band, the instrumental magnitudes can be accurately transformed to $\mathrm{V}$ magnitudes using a simple color term. This is possible because the RRLS span a fairly narrow range in color, even as they vary in color during their pulsations.

The raw data of the variability survey are automatically processed by the Yale Photometric Pipeline, as soon as they start arriving every morning. This pipeline produces a catalog of positions and instrumental magnitudes for all objects above a detection threshold. The software starts with the standard tasks of using the nightly flats and darks to do background subtraction and flatfielding of the image. The preprocessed data are then run through SExtractor, a standard Source Extraction program (Bertin \& Arnouts 1996). In the RR Lyrae study we used aperture photometry with a 6 pixel diameter apertures. Instrumental magnitudes were calculated as 
$\mathrm{m}=25.0-2.5 \log ($ flux in aperture $)$

where the flux in the aperture was in units of analog to digital converter units (ADU). Astrometric calibration was done using the USNO-B1.0 catalog as the reference. The precision of the resulting astrometry was a few tenths of an arcsec in the star positions.

\section{Selection and Calibration of the RR Lyrae Sample}

\subsection{Selection of RR Lyrae Candidates}

Previous RRLS surveys of the halo (Kinman et al. 1966; Vivas et al. 2004) have shown that RRLS are relatively rare objects on the sky, typically few per square degree. This is a consequence of the low density of the halo, the relatively short time-scale of the RRLS phase, and the fact that the evolutionary paths of some horizontal branch (HB) stars never enter the instability strip. RRLS can be separated from most other kinds of stars by their variability, but to make this manageable, it is very useful to limit the possible candidates on the basis of color. Here we make use of $\mathrm{u}-\mathrm{g}$, g-r colors from the Sloan Digital Sky Survey (SDSS), as have, for example, Ivezić et al. (2005); Watkins et al. (2009); Sesar et al. (2010).

The top diagram in Figure 3 shows for a small fraction of our survey area the locations of stars in the plane defined by the dereddened colors $(u-g)_{0}$ and $(g-r)_{0}$. The values of the interstellar extinctions that the SDSS lists for each star were used to remove the reddenings. The large number of stars lying across the top of the diagram are part of the locus of main-sequence stars (see Ivezić et al. 2005, for a larger view). The stars scattering below at smaller values of $(g-r)_{0}$ include RRLS. This is illustrated in the middle diagram in Figure 3, where we have plotted the colors of 441 RRLS from the QUEST survey (Vivas et al. 2004) that we identified in data release 7 (DR7) of the SDSS survey. The hexagon that is plotted in all three diagrams of Figure 3 encloses the area that Ivezic et al. 
(2005) recommended for finding RRLS candidates based on a smaller number of QUEST RRLS. We found that a bigger region (see Figure 3) yields a more complete sample of RRLS. In addition, one corner of the hexagon extends into the region occupied by many main-sequence stars. While there is indeed some overlap between RRLS and the main sequence in this plane, we found that a color selection that is based on the hexagon returns a huge number of main-sequence stars, a significant fraction of which are variable but not RRLS. With little loss of completeness, we found that cutting off the corner of the hexagon greatly speeded up our survey. The search box that we adopted is shown as solid lines in Figure 3, which encloses $98 \%$ of the RRLS from the QUEST survey. The colors of the stars in our catalogue of RRLS are plotted in the lower diagram of Figure 3, where one can see that a few stars lie outside our search box, but inside the hexagon. This is a consequence of starting the survey with the hexgon and later switching to the larger region and repeating the areas previously covered.

With our 60s exposures, stars brighter than $r=14$ saturate most of the CCDs in the camera, and stars fainter than $\mathrm{r} \sim 20$ produce low signal to noise images. Consequently, we imposed r magnitude limits of 14 and 20 when selecting candidate RRLS from the SDSS photometric catalog. A query of the SDSS database for stars that have the proper colors to the RRLS candidates yielded more than 29,000 objects in the region of the sky covered by this first band of the LSQ survey. The LSQ database was then searched for these stars, and for each one found, a separate catalogue of observations was compiled. After experimenting with known RRLS, we found that 12 or more observations were required before a RRLS could be identified with some confidence. The initial set of candidates was then reduced to the $\sim 24,000$ that have at least 12 observations.

The area of the sky that is covered by this first part of the LSQ survey is shown in Figure 4. Since we select our candidate RRLS on the basis of their $(u-g)_{0}$ and $(g-r)_{0}$ 
colors, the survey area is the SDSS footprint between 10 and 14 hrs $\left(150.0^{\circ}-210.0^{\circ}\right)$ in right ascension $(\alpha)$ and $-10^{\circ}$ and $+10^{\circ}$ in declination $(\delta)$. In galactic coordinates $(1, b)$, the survey has a fan-like geometry that spans $230^{\circ}<l<350^{\circ}$. At $l \sim 240^{\circ}$, the range in $\mathrm{b}$ is $\sim 40^{\circ}-60^{\circ}$. Near the middle of region $\left(l \sim 290^{\circ}\right)$, the range is $\sim 58^{\circ}-\sim 72^{\circ}$, and at $l \sim 330^{\circ}$ it is $\sim 57^{\circ}-\sim 70^{\circ}$. The survey region, which covers $\sim 840 \mathrm{deg}^{2}$ of the sky, contains parts of the leading stellar stream from the Sgr dSph galaxy, the Virgo Overdensity (VOD), the Virgo Stellar Stream (VSS), the stellar stream detected by (Walsh et al. 2009; Jerien et al. 2013), and several irregularies in the density distributions of halo tracers (see Keller et al. 2008, 2009; Keller 2010) that may be additional halo substructures.

Plotted in the top diagram of Figure 4 is the entire sample of RRLS candidates that were identified in SDSS DR7. The gaps between the rows of CCD's, the loss of several CCD's in the camera, and the variable weather conditions all conspired to cause some candidate RRLS to be under observed. The bottom diagram shows the locations of the stars for which the LSQ survey obtained fewer than 12 observations. It shows that these stars, which for our purpose were essentially unobserved, are scattered throughout the region, with some concentration in narrow bands in $\delta$. The stars for which we did obtain 12 or more observations, the "observed stars", constitute $80 \%$ of all the RRLS candidates.

For these observed stars, we plot in Figure 5 the fraction of the sample having numbers of observations $\left(N_{\text {obs }}\right)$ less than a given number N. One can see from this diagram that $90 \%$ of the sample of stars have $>35$ observations and $50 \%$ have $>60$ observations. We discuss below comparisons between the LSQ survey and previous ones in the same region, which suggest that the very few RRLS are missed by the LSQ survey if $N_{\text {obs }}>30$. 


\subsection{Relative Photometry}

Before identifying the RRLS in the sample of candidates, we performed relative photometry in order to remove the variations in the instrumental magnitudes that are produced by the different sensitivities of the CCD's and the variable atmospheric extinction and transparency. For each observation of a candidate RRLS, we saved in a "standard star" file the photometry of all point-sources on the same chip that were more than 6 pixels from the edge of the CCD and had instrumental magnitudes in the range 12-18, which corresponds to $\mathrm{V} \sim 14.2-20.2$. This standard star file was pruned of objects that contained any bad pixels within a $6 x 6$ pixel box centered on the object. One observation was selected as the "reference observation" to which all others would be adjusted by differential photometry of the standard stars. To select the reference observation, we first identified the CCD on which there were the most observations of the target with very reliable astrometry, defined as having a match of more than 50 stars with the USNO-B1.0 catalog. Among these observations from the same CCD, we selected as the reference observation the one that had the brightest instrumental magnitudes of the USNO stars, which is likely to be the one that was obtained with the smallest atmospheric extinction and the highest transparency. Because the observations from different CCD's could not be as precisely calibrated as the ones from the same CCD, this procedure maximized the number of well-calibrated observations. To determine the offset between an observation and the reference one, we selected the standard stars that lie within 6 arcmin of the candidate RRLS. The median of the differences in magnitude of these standard stars between the observation and the reference observation was used as the first estimate of the magnitude offset. To remove distant outliers, which may be variable stars, the stars that were more than 6 times the errors in their magnitudes from the median were clipped from the sample. As the final offset, we adopted the mean of the magnitude differences, weighted by the errors in the magnitudes, of the stars that remained after the clipping. This procedure 
yielded magnitude offsets with precisions of typically 0.008 mag, which when applied to the observations of the candidate RRLS, removed their zero-point differences. A file was created for each candidate RRLS, which contained the modified Julian Date of the observations, the zero-point adjusted instrumental magnitudes and their errors, flags produced by SExtractor that indicated a potential problem with the measurements, and a flag indicating the star was within 6 pixels of the CCD edge. While not all of the flagged observations turned out to deviate substantially from non-flagged ones, the flags were useful for identifying the origin of a few very deviant points from the mean magnitude of a constant star or from an otherwise well-determined light-curve of a variable star.

\subsection{Identification of RRLS}

Our methods for separating RRLS from non-variable stars and other types of variable stars closely follow the techniques that we developed for the QUEST RRLS survey (Vivas et al. 2004). For each candidate RRLS found in the LSQ database, the mean instrumental magnitude and root-mean-square (rms) of the variations around this mean were calculated. Figure [6 shows a plot of the rms values against a rough V magnitude, which was obtained by addition of the average zero-point offset of 2.2 to the instrumental magnitudes. The majority of the stars define a sequence that is roughly constant at rms $~ 0.02$ over the range $13.9<V<17.2$. Fainter than 17.2, this sequence broadens and

rises to $\mathrm{rms} \sim 0.12$ at $\mathrm{V} \sim 20.4$. To remove constant stars and very low amplitude variables from consideration, we examined for periodicity only those stars whose rms values lie above the curve in Figure 6, which is constant at 0.05 for $V<17.2$ and is a quadratic function of $\mathrm{V}$ for $V>17.2$. The RRLS that we have identified in this survey are also plotted in Figure 6 as the large symbols, and their locations suggest that the lower limit that we have set on rms has not excluded many RRLS from further analysis. In fact, we had originally 
set the rms limit 0.01 mag larger. Lowering it to the level shown in Fig. 6 yielded $\sim 1000$ more candidates, but only 3 of these passed our criteria for RRLS.

To determine if a candidate is an RRLS, we used the period-finding method of Lafler \& Kinman (1965) (see also Vivas et al. 2004; Saha \& Hoessel 1990). The measurements for each candidate were phased with several thousand trial periods in the range $0.15 \leq P \leq 0.9$ days. For each trial period, the observations were sorted by increasing phase and the parameter $\Theta$ was calculated. $\Theta=\sum_{i}\left(m_{i}-m_{i+1}\right)^{2} / \sum_{i}\left(m_{i}-\bar{m}\right)^{2}$, where $m_{i}$ and $\bar{m}$ are the individual magnitudes and the mean magnitude, respectively. If a trial period produces a light-curve rather than a scatter plot, $\Theta$ will have a relatively small value. To identify values of $\Theta$ that are potentially interesting, the parameter $\Lambda$ was also calculated for each trial period. $\Lambda=\bar{\Theta} / \Theta$, where $\Theta$ is the value obtained with the trial period and $\bar{\Theta}$ is the average value of $\Theta$ obtained with all trial periods. A large value of $\Lambda$ indicates that the trial period produces a well-determined light curve. A lower limit on $\Lambda$ was set after experimenting with successively lower values and seeing if any potential RRLS were missed. If the largest value of $\Lambda$ that was obtained with the set of trial periods was less than 2.2, the star was excluded from further analysis. If $\Lambda$ was greater than 2.2 , then the light-curves produced by the 3 trial periods that yielded the largest values of $\Lambda$ were examined by eye to see if any had the characteristics of either a type ab (fundamental mode pulsator) or a type c (first harmonic mode). In the QUEST survey, the threshold on $\Lambda$ was set at 2.5. We have lowered it here because the set of LSQ measurements for some stars included a few very deviant magnitudes. The otherwise reasonable light curve for a RRLS star could have an anomalously low value of $\Theta$ because of deviant points, which are easily seen if the light curves are examined by eye. By setting the threshold at 2.2, the light curves of many more stars that are not RRLS had to be examined, but it is less likely that any true RRLS were missed. 
The periods producing these 3 best light-curves were almost always aliases of each other. In most cases, the light-curve with the largest value of $\Lambda$ was unquestionably that of a RRLS or another type of variable (e.g., W UMa eclipsing binary). In some cases, there was some ambiguity as to whether the $1^{\text {st }}$ or $2^{\text {nd }}$ best light-curve was the correct one. If it appeared likely that the star is a RRLS, the well-known correlations among period, amplitude, and light-curve shape were used to decide between the two periods. However, in cases where there were fewer than 25 observations, it was not always clear what was the best choice between the alias periods. For $<1 \%$ of the RRLS, it was decided that the period that gave the third largest value of $\Lambda$ was the correct one.

Once a star was identified as a RRLS, the heliocentric Julian Dates of the observations were calculated, and template light-curves from Layden (1998) or from Mateu et al. (2009), for the types ab and c respectively, were fit to the data. Allowing the period and the time of maximum light to vary around the values obtained from the best trial period, the $\chi^{2}$ of the deviations of the data from the templates were minimized, which produced the best estimates of the period, amplitude, and Heliocentric Julian Date of maximum light. The magnitudes of each template were translated to intensity units, integrated, and the mean was translated back to magnitude units, which yielded the mean instrumental magnitude, $\left\langle V_{\text {Inst }}\right\rangle$, which was later transformed to $\langle V\rangle$ by the calibration that is described below. We did not attempt to subdivide the type c variables into the type $\mathrm{d}$, the multimode variables, or type e, the suspected $2^{\text {nd }}$ harmonic pulsators (Alcock et al. 1996), because this does not affect their use as standard candles to probe the halo.

For $\sim 20 \%$ of the RRLS, the light-curve produced by the best trial period had a few points that were clearly very deviant from the majority. In most cases, these points had significantly larger photometric errors than the majority, suggesting that they were measured from observations with high backgrounds from Moon-light or through thin clouds. 
In some cases, the photometric errors of the deviant points were small compared to their deviations from an otherwise well determined light-curve. The origin of these deviations are unknown. They could be due to measurements too near an edge of a CCD or from behind the joint between the two halves of the filter. In all cases, these deviant points and the ones with large photometric errors had little effect on the identification of a RRLS or the determination of its period. They were deleted from the sample before the template fitting

process to ensure that they did not affect the determination of the best period and $\left\langle V_{\text {Inst }}\right\rangle$.

\subsection{Absolute Calibration}

The next step in the analysis was to transform $<V_{\text {Inst }}>$ to $\langle V\rangle$ for each RRLS. The observation that was selected as the reference image for the differential photometry was calibrated to V using the photometry of the SDSS DR7 catalogue. Field stars were selected within a circle of 300 arcsec on the reference image of each RR Lyrae star. The SDSS g and $\mathrm{r}$ magnitudes of these stars were then converted to the Johnson $\mathrm{V}$ magnitudes by the relation (Lupton 2005) :

$$
V=g-0.5784(g-r)-0.0038
$$

The mean difference between these magnitudes and their LSQ instrumental magnitudes define a zero-point offset. To examine the accuracy of this technique, we used the same method on 104 stars that are listed by Stetson (2000) as V standards. These stars, which have $14.75<V<18.75$, were selected on the basis of being far from CCD saturation, having good S/N, and having no near neighbors. Given the broad width of the LSQ filter, we were not surprised to find that there was a residual offset that depended on the colors of the stars. Consequently, we adopted the procedure of removing stars that were very different in color from RRLS from the samples of calibrating SDSS stars, and then added 
the following small correction to the $\mathrm{V}$ mags obtained from the Lupton equation before computing the zero-point offset:

$$
\Delta V=0.1213(g-r)-0.0681
$$

The success of this technique is evident in Figure 7, where we plot for the Stetson standards the difference between the Stetson V mag and the V mag obtained from our calibration of the LSQ observations. This distribution is well fit with a Gaussian distribution with a mean of zero and a sigma of 0.03 mag. Another estimate of the precisions of our measurements of $\langle V\rangle$ for RRLS is provided by the comparisons between our survey and previous ones for the same stars (see below).

The filter that is used in the LSQ survey is physically two identical filters that butt together. We could not calibrate well the observations obtained with the CCDs that lie behind the filter joint, although we could measure the periods and the light-curves of the RRLS observed with them. Because of their uncertain calibration, these stars are labeled with flag $=1$ in Table 1 . A comparison with the QUEST survey (see below) suggests that the calibration for these stars has a sigma of $\sim 0.2 \mathrm{mag}$. The well-calibrated stars have flag $=0$ in Table 1.

Table 1 lists the RRLS by increasing $\alpha$. The missing numbers are stars that we believe are Anomalous Cepheids in the Sextans dSph galaxy (see below), which are listed in Table 2. In the "Notes" column of Table 1, the RRLS that are likely members of the Sex dSph galaxy are identified, and the variable number is given if the star is in the study by Mateo et al. (1995). For each star in Tables 1 and 2, Table 3 lists the heliocentric Julian Date of observation, the observed V magnitude transformed to the standard system, and its standard deviation as given by photon statistics. While the uncertainty in the calibration to the standard system is larger, in general, that these standard deviations, they provided a means for weighting the observations when fitting the template light-curves. 
In Figure 8, a few typical light curves for type ab and type c variables are displayed on the left and right panels, respectively. On the top row are two stars that have mean magnitudes near the middle of the range of the LSQ survey and typical numbers of observations $(\sim 50)$. The middle row shows two stars near the faint limit of the survey, again having typical numbers of observations. Plotted in the bottom row are two stars that have very few observations $(<18)$, and yet their light curves are reasonably well determined.

\subsection{Comparisons with previous RRLS surveys}

In this section, we compare our survey with six previous RRLS surveys that overlap to some degree. The comparisons made here are important check on our detection procedures and our photometric precision.

\subsubsection{The QUEST Survey}

The LSQ survey includes the region within $150^{\circ} \geq \alpha \leq 210^{\circ}$ that was covered by the QUEST survey, which employed an earlier QUEST camera on the $1 \mathrm{~m}$ Schimdt telescope of the Llano del Halo Observatory in Venezuela. The original survey covered $380 \mathrm{deg}^{2}$ of the sky in a $2.3^{\circ}$ wide band centered on $\delta=-1$ (Vivas et al. 2004) and $\alpha$ in the ranges $60^{\circ}-90^{\circ}$ and $120^{\circ}-255^{\circ}$. Later a second band at $\delta=-3^{\circ}$ and $\alpha$ in the ranges $0^{\circ}-90^{\circ}$ and $120^{\circ}-195^{\circ}$ was added using the same procedures (Vivas et al. 2006). Some of the RRLS in the original catalogue have been re-observed to improve their light-curves (Vivas et al. in preparation). The QUEST survey used a $\mathrm{V}$ filter, and its measurements were tied to the standard V system.

In the region of overlap, 223 were identified as RRLS in either the first or 2 nd bands of the QUEST survey. A few other QUEST RRLS in the same area of the sky are not included 
in this comparison because they are either brighter than the saturation limit of the LSQ survey or are probably not RRLS on the basis of their SDSS colors and/or a reexamination of their light-curves. Of the 223 QUEST RRLS, 178 or $80 \%$ were rediscovered as RRLS by the LSQ survey. Nineteen of the 45 stars that were missed had fewer than 12 LSQ observations, and all but 2 had fewer than 30. The QUEST survey is itself incomplete, however (Vivas et al. 2004). In the region of the sky where the LSQ and QUEST surveys overlap, the LSQ survey identified 283 RRLS, of which 178 are in common with the QUEST survey. Thus, the minimum number of RRLS in this region is 328, and the LSQ and QUEST surveys found $86 \%$ and $68 \%$ of these RRLS, respectively. Many of the RRLS that the QUEST survey missed are near its faint limit, which is consistent with its estimated completeness (Vivas et al. 2004).

Of the 178 LSQ RRLS in common with QUEST, 158 have good quality zero-point calibrations (Flag $=0$ in Table 1 ). For these stars, the mean difference in $\langle V\rangle$, in the sense LSQ-QUEST, is 0.021 with a standard deviation $(\sigma)$ of 0.061 . The upper diagram of Figure 9 contains the histogram of the differences, which is compared to a Gaussian distribution that has the same mean and $\sigma$. The zero-point uncertainty of the QUEST magnitude system is 0.02 , and in the QUEST catalog the errors in $\langle V\rangle$ stemming from the light-curve fitting average about 0.05 mag (Vivas \& Zinn 2006). Because additional observations have been added more recently for several stars and the $\langle V\rangle$ values have been updated, the average error of the QUEST magnitudes may be 0.04 . With this estimate, the agreement between the QUEST and the well-calibrated LSQ measurements of $\langle V\rangle$ suggest that the errors in the latter are also $\sim 0.04$ mag.

For 20 of the stars in common with QUEST, only a very rough calibration could be made of the LSQ magnitudes (flag $=1$ in Table 1 ). The lower diagram in Figure 9 shows the distribution of the differences in $\langle V\rangle$ between LSQ and QUEST, which is compared with 
a Gaussian distribution that has the same mean (0.006) and $\sigma(0.21)$. The poorly calibrated LSQ measurements have as expected large uncertainties in their mean magnitudes, and this compromises to some extent their use as standard candles. The identification of these stars as RRLS, and their periods, times of maximum light, and amplitudes are not sensitive to this uncertainty.

A comparison of the LSQ periods and the QUEST periods indicated very little difference for most stars $\left(|\Delta P|<1 x 10^{-3}\right.$ day). However, 5 stars that are identified here as long-period, low-amplitude type ab (more precisely type b), QUEST had identified as type c. In each case, the two periods are aliases of each other, and the one selected here gives better results than the QUEST period with the LSQ data and also excellent light-curves with the QUEST data. According to the LSQ observations, another QUEST star (Q119) is an Anomalous Cepheid in the Sextans dSph galaxy, with a period of about 2 days (see below). The period found here also fits the QUEST observations.

\subsubsection{The sample of Wu et al. (2005)}

Wu et al. (2005) obtained unfiltered CCD photometry for 69 stars that were identified as candidate RRLS in the commissioning data of the SDSS by Ivezić et al. (2000). They confirmed that these stars are RRLS and measured their periods and light-curves.

In the region of the sky covered by the LSQ survey, Wu et al. (2005) measured the light curves and periods of 42 stars. Thirty-two of these stars were identified as RRLS by the LSQ survey, and one was rejected as a probable W UMa eclipsing variable (\#29 in Wu et al. 2005, which is 211 in the QUEST survey, Vivas et al. 2004). The recovery rate of RRLS is therefore $78 \%$. All of the missed RRLS had fewer than 20 LSQ observations, and 7 of the 9 had fewer than 10 observations. The periods derived here are in excellent agreement 
with the ones obtained by Wu et al. $\left(|\Delta P|<1 \times 10^{-3}\right.$ day). Their unfiltered measurements were calibrated to r' band with a precision of $0.07 \mathrm{mag}$, and the transformation between $\left\langle r^{\prime}\right\rangle$ and $\langle V\rangle$ was uncertain by $0.1 \mathrm{mag}$. because measurements of mean color were not

obtained (Wu et al. 2005). Consequently, we have not made a comparison between the mean magnitudes because it would carry much less weight than the ones involving the QUEST survey and the ones below.

\subsubsection{The LONEOS-I RRLS survey}

Miceli et al. (2008) used the data from the Lowell Observatory Near Earth Objects Survey Phase I (LONEOS-I) to identify 838 ab type RRLS in a $1430 \mathrm{deg}^{2}$ area of the sky, which partially overlaps with the LSQ survey. Their photometry is tied to a synthetic SDSS-based V magnitude with a zero-point scatter of 0.13 mag. There are 16 LONEOS-I RRLS in the region of overlap and fainter than the bright magnitude limit of the LSQ survey. The LSQ survey recovered 14 or $88 \%$ of these stars. For the two stars that were missed, the LSQ survey did not obtain observations at their positions, probably because the stars either fell on the gap between two CCDs or on a dead CCD. For 13 of the 14 stars in common, the LSQ magnitudes are well calibrated. The mean difference in $\langle V\rangle$, in the sense LSQ-LONEOS-I, is 0.024 with $\sigma=0.139$. These differences are consistent with the estimate of the errors in the LSQ magnitudes that we made above from the comparison with QUEST, and the estimate made by Miceli et al. (2008) of the zero-point uncertainty in the LONEOS-I measurements. The periods obtained for 14 stars in common are in good agreement $(|\Delta P|<2 \times 10-3)$. 


\subsubsection{The SEKBO survey}

Using the data from the Southern Edgeworth-Kuiper Belt Object (SEKBO) survey, Keller et al. (2008) selected a large number of candidate RRLS based on color and variability in typically 3 observations. Later, Prior et al. (2009) obtained sufficient observations to construct light-curves for a subsample of these stars, and 7 lie in the region covered by the LSQ survey. Only 3 (43\%) were recovered by the LSQ survey. Fewer than 10 observations were obtained for three of the missed stars. Although 24 observations were obtained of the remaining star, which is usually sufficient for a good quality light curve, the LSQ observations are noisy, and a firm identification as a RRLS could not be made. For one of the RRLS in Prior et al. (2009; 107434-394 = LSQ152), their period (0.327 day) from only 8 observations appears to be incorrect. The 77 LSQ observations indicate that this star is a type ab with a period of 0.65578 day. The periods of the other two stars in common are in good agreement, and for these two stars the mean difference in $\langle V\rangle$, in the sense LSQ-Prior et al., is -0.01 with $\sigma=0.07$, which are consistent with errors in the measurements.

\subsubsection{RRLS and Anomalous Cepheids in the Sextans dSph galaxy}

The Sextans dSph galaxy lies near the southern boundary of our RRLS survey at $\alpha=153.260$ and $\delta=-1.620$. Its distance, $86 \pm 4 \mathrm{kpc}$ (Mateo 1998), places it at the extreme limit of the LSQ survey. The positions of the stars that we identify here with Sextans are within the tidal radius of its center, according to the measurements by Irwin \& Hatzidimitriou (1995). Since halo RRLS of their $\langle V\rangle$ are very rare, this is strong evidence for membership in the dSph galaxy. Our requirement that the candidate RRLS from the SDSS have $r<20.0$ undoubtedly removed some Sextans RRLS from possible inclusion in our sample. While we intend later to make a more thorough survey of Sextans 
without this limitation, we note for now that our sample includes 7 RRLS that are probably members (Table 1). It also includes 6 stars that are known or likely Anomalous Cepheids (AC). The AC are listed in Table 2 and discussed briefly below.

One RRLS and $3 \mathrm{AC}$ in our sample of Sextans variables were observed previously by Mateo et al. (1995). The mean difference in $\langle V\rangle$, in the sense LSQ-Mateo et al., is 0.00, with $\sigma=0.05$. Since these stars are among the very faintest in our sample, it is encouraging that the agreement is this good. For one of the AC variables (LSQ34 = V34, Mateo et al.), our observations are better fit with a period of 0.50640 day than with the period of 0.34126 that is listed by Mateo et al. (1995). For the other three stars in common, the periods are in agreement $\left(|\Delta P|<2 \times 10^{-3}\right)$. The light-curve of RRLS LSQ38 (V3 in Mateo et al. 1995) is shown in Figure 8 .

In Table 2, we have listed the 6 probable $\mathrm{AC}$ variables, 3 of which are in common with Mateo et al. (1995). Their absolute $\mathrm{V}$ magnitudes, $M_{V}$, were calculated using an apparent distance modulus of $(m-M)_{V}=19.76$ for Sextans (Mateo 1998). According to the relationships given in Pritzl et al. (2002), these stars lie on either the P-L relationship for fundamental or first harmonic AC variables. LSQ 40 is noteworthy because its $\sim 2$ day period is one of the longest periods of the $\mathrm{AC}$ variables in the $\mathrm{dSph}$ galaxies.

\subsubsection{The Catalina Surveys}

The recent large surveys for type ab RRLS using the data from the Catalina Surveys Data Release 1 became available as our analysis was nearing completion (Drake et al. 2013a.,b). These surveys, which cover a large area of the sky, include the region studied here. The Catalina surveys used three telescopes, each equipped with similar CCD detectors without filters. Their calibration methods for RRLS yielded measurements of $\langle V\rangle$ with an 
accuracy of $\sim 0.05 \mathrm{mag}$ (Drake et al. 2013a). A comparison of the positions of the stars in the LSQ and Catalina surveys yielded a total of 791 stars in common, 790 RRLS and one $\mathrm{AC}$ in the Sextans dSph galaxy (LSQ36 = V1 in the study by Mateo et al. 1995).

There is generally good agreement between the periods determined by the LSQ and Catalina surveys. With the exception of 4 stars, $|\Delta P|<7 \times 10^{-3}$ day, with an average of $2.4 \times 10^{-4}$. For one (LSQ611) of the 4 deviant stars, LSQ survey had obtained only 11 good observations. This star was correctly identified by the LSQ survey as a type ab RRLS, but the wrong period was selected from the two that produced equally good light curves. The other period agrees with one obtained by the Catalina survey, and we have adopted it for the LSQ catalogue (Table 1). The other 3 deviant stars (LSQ360, 522, 953) are classified as type ab RRLS by the Catalina survey, but the LSQ survey indicates that they have the periods, amplitudes, and light-curve shapes of type c variables. We have retained the LSQ classification for the LSQ catalogue.

For 730 stars in common, the LSQ measurements have good photometric calibrations (Flag $=0$ in Table 1$)$. The mean difference in $\langle V\rangle$ in the sense LSQ-Catalina is 0.07 with a standard deviation of 0.11 . Some of this discrepancy is probably due to image blending, which affects differently the two surveys. For example, 22 of the stars in common differ in $\langle V\rangle$ by more than 0.3 mag. For 19 of these stars the deviations are positive, indicating that the Catalina surveys measured brighter magnitudes. An inspection of the SDSS images for these stars suggests that the measurements for 17 of them may be affected by the presence of nearby stars or galaxies. One of the 3 stars with a large negative offsets in magnitude is a relatively bright star (LSQ1063), which is near the saturation limit of the LSQ survey. The LSQ measurements of it are therefore suspect. There is no ready explanation for the other 2 stars with large negative deviations or for the 2 stars with large positive ones that appear to be unblended. The measurements for some of the stars that deviate by smaller 
amounts may also be affected by image blending.

For 61 of the stars in common between the LSQ and Catalina surveys, only a poor photometric calibration was possible for the LSQ observations (Flag $=1$ in Table 1 ). The mean difference in $\langle V\rangle$, in the same sense as above, for these stars is 0.06 mag with a standard deviation of $0.22 \mathrm{mag}$. An almost identical standard deviation was found above in the comparison between the LSQ and QUEST surveys.

To investigate the completeness of the LSQ survey, we restricted the overlap region with the Catalina surveys to $\delta \geq-1^{\circ}$, where the rectangular geometry of the LSQ survey

simplified the comparison. The LSQ survey is seriously incomplete for $\langle V\rangle \leq 14.0$ because of saturation of the CCDs, and both surveys are incomplete fainter than $\langle V\rangle=20.0$. Within $14 \leq\langle V\rangle \leq 20$ and for $10^{\circ} \geq \delta \geq-1^{\circ}$, the Catalina surveys identified 859 type ab variables and the LSQ survey found 766 type ab and 267 type c RRLS, of which 615 are in common with Catalina. The LSQ survey therefore found $72 \%$ of the Catalina type ab RRLS. Of the ones that the LSQ survey missed, only $15 \%$ had the 12 or more observations that were required were before a period search was undertaken, and only $7 \%$ had more than 30 observations, which is usually adequate for a good quality light-curve. The LSQ data for these relatively well-observed stars indicated that they were variable stars, but the data were either too noisy or too poorly spaced in phase for a firm decision to be made about the type of variable.

\subsection{Completeness of the Survey}

The completeness of the LSQ RRLS survey can be estimated from the overlap with the previous surveys. As noted above, for $80 \%$ of the RRLS candidates sufficient observations were obtained for a period search (i.e., $N_{o b s} \geq 12$ ), and our color selection box includes $98 \%$ 
of the QUEST RRLS. The above comparisons between the LSQ survey and the previous ones indicate that some additional RRLS were missed, primarily because of small $N_{o b s}$. For stars with $N_{o b s} \geq 12$, the LSQ survey found $\sim 92 \%$ of the RRLS that were identified by the other surveys (exluding the survey of the Sex dSph galaxy, which only contains stars at the faint limit of the survey). If this is representative of the whole survey region, the LSQ survey contains $\sim 70 \%$ of the total population of RRLS $(\sim 0.80 \mathrm{x} 0.98 \mathrm{x} 0.92)$.

To examine the completeness of the LSQ survey as a function of $\langle V\rangle$, we show in the top diagram of Figure 10 the magnitude distribution of the 223 QUEST RRLS in the region of overlap and the distribution for the 178 that were recovered by the LSQ survey. This diagram illustrates that $\gtrsim 70 \%$ of the QUEST RRLS were recovered over the magnitude range $14 \leq\langle V\rangle \leq 19.5$. Only one QUEST RRLS in the overlap region is fainter than 19.5, and it was missed by the LSQ survey. In the lower diagram in Figure 10, we show a similar plot for the 859 type ab RRLS identified by the Catalina surveys in the region of overlap with $\delta \geq-1^{\circ}$ and the 615 RRLS recovered by the LSQ survey in that region. With the exceptions of the two brightest bins, this diagram is consistent with top one in showing that the completion of the LSQ survey is $\gtrsim 70 \%$ for $\langle V\rangle \leq 19.5$.

The variables in the Sex dSph galaxy provide additional information on the completion of the LSQ survey near its faint limit. Of the 42 RRLS and AC variables in the study by Mateo et al. (1995), 34 are RRLS in the narrow interval $20.25 \leq\langle V\rangle \leq 20.60$. The LSQ survey did not recover any of these variables. The LSQ survey did recover 4 of the 8 remaining variables that span the range $18.88 \leq\langle V\rangle \leq 20.25$, and it found 6 new RRLS in the dSph galaxy with $20.0 \leq\langle V\rangle \leq 20.5$. This and Figure 10 suggest that the completeness of the LSQ survey is $\gtrsim 70 \%$ for $15.0 \lesssim\langle V\rangle \leq 19.5$, but then falls to zero over the range $19.5 \leq\langle V\rangle \leq 20.6$

Because the CCD's in the LSQ camera have different sensitivities and saturation 
limits, it is likely that the completeness at $\langle V\rangle>19.5$ and $\lesssim 14.5$ varies with position on the sky. For RRLS with small interstellar extinctions, as is the case in this region of the LSQ survey, $\langle V\rangle \sim 19.5$ corresponds to a distance from the Sun, $d_{\odot}$, of $\sim 60 \mathrm{kpc}$. In the following discussion of the density profile of the halo, we do not use the densities of RRLS at $d_{\odot}>60 \mathrm{kpc}$ in calculations nor do we compute densities for stars with $\langle V\rangle<14.55$.

\section{The spatial distribution of the RR Lyrae variables}

To determine the distances of the RRLS, we have adopted an absolute magnitude of $M_{V}=+0.55$ for all stars. This value, which we used earlier in the QUEST survey (Vivas \& Zinn 2006), is in agreement with most $M_{V}-[\mathrm{Fe} / \mathrm{H}]$ relations for RRLS near the middle of the $[\mathrm{Fe} / \mathrm{H}]$ range of halo RRLS, i.e., $[\mathrm{Fe} / \mathrm{H}] \sim-1.6$, including the recent determination by statistical parallax for type c variables (Kollmeier et al. 2013). For the interstellar extinction, we adopted the values given by the dust maps of Schlegel et al. (1998). For the well-calibrated stars in the LSQ survey (Flag $=0$ in Table 1), the fractional error $\left(\sigma_{d_{\odot}} / d_{\odot}\right)$ is about 0.07 (see Vivas \& Zinn 2006). For the poorly calibrated stars (Flag $=1$ ), the fractional error is $\sim 0.11$, which is similar to the distance uncertainties for halo main-sequence stars (e.g., Bonaca et al. 2012).

The distribution of the LSQ sample of RRLS as a function of $\alpha$ and $d_{\odot}$ is shown in Figure 11, where one can readily see the leading stream from the Sgr dSph galaxy and the Sex dSph galaxy. While the overdensity due to the Virgo Stellar Stream (VSS) can be recognized in this diagram, its extent becomes clearer once the background of RRLS is estimated. To do this for the LSQ RRLS, we first calculate the variation of their number density with $R_{g c}$. Since the form of this density profile provides a clue to the formation of the halo, it is worthy of investigation on its own. Recent studies have confirmed older ones that suggested that the halo is flattened in the same direction as the Galactic disk and have 
also greatly strengthened the case made first by the RRLS survey by Saha (1985) that there is a significant break in the power-law slope of the density profile, e.g., Sesar et al. (2011), Deason et al. (2011). We find evidence for both of these effects here.

\subsection{The number density profiles}

The sample of RRLS is sufficiently large that it is possible to subdivide it and still have sufficient numbers of stars in each subregion to determine the density profile. We can then investigate the variation of the density profile with direction. Because the geometry of the survey region is simpler in equatorial coordinates than galactic ones, we divided the survey area into 12 strips, each $5^{\circ}$ wide in $\alpha$. These regions vary somewhat in $\delta$ (see Figure 4), which was taken into account when calculating the number density ( $\rho$ in units of $\# k p c^{-3}$ ) of RRLS. We also tried wider strips in $\alpha$ with subdivisions by $\delta$ and did not find any significant changes in the profiles. To avoid sample incompleteness due to either the saturation of the CCDs or the bright cutoff in the candidate selection, we excluded the very brightest RRLS when calculating the number densities. The number densities were calculated in bins in distance modulus, $\left(\langle V\rangle-M_{V}\right)_{0}$, starting at 14.0. The width of the first bin was 1.0 mag to ensure that the volume was sufficiently large that some RRLS were included. Subsequent bins were 0.5 mag wide. Since the first eight bins only include stars with $d_{\odot} \lesssim 50 \mathrm{kpc}$, they should be immune from the incompleteness near the sample's faint limit (see above), and only these bins were used when fitting a function to the number densities. The plots of the number densities include more distant bins because it is interesting to see how they compare with extrapolations of the fitted functions.

One can see from Figure 11 that the subdivisions by $\alpha$ will be affected differently by the major substructures in the region. Obviously, the Sgr stream will have a profound effect on the profiles for $\alpha \gtrsim 185^{\circ}$. The VSS is expected to have a smaller but nonetheless 
significant effect on the profiles for $180^{\circ} \lesssim \alpha \lesssim 190^{\circ}$. Because the region $150^{\circ} \leq \alpha \leq 170^{\circ}$ appears to be devoid from major substructures, it was chosen for estimating the density profile of the smooth component of the halo, but first the RRLS from the Sex dSph galaxy were excluded.

As others have in the past (e.g., Preston et al. 1991; Vivas \& Zinn 2006), we adopt either a spherical halo or an ellipsoidal one that is flattened towards the galactic plane. For the density profile, we adopted $\rho(R)=\rho_{\odot}\left(R / R_{\odot}\right)^{n}$, where $\mathrm{R}$ is either the radius of the sphere or the semimajor axis of the ellipsoid, and $R_{\odot}$ and $\rho_{\odot}$ are the distance of the Sun (set to 8 kpc, e.g., Majaess 2010) and the density at the Sun's position, respectively. The logarithmic form of this equation (eq. 1) was used to make weighted least squares fits to the densities in the different bins of $\left(\langle V\rangle-M_{V}\right)_{0}$. For a spherical halo, which we will consider first, $R=R_{g c}=\left(X^{2}+Y^{2}+Z^{2}\right)^{1 / 2}$, where $\mathrm{X}, \mathrm{Y}$, and $\mathrm{Z}$ are cartesian coordinates with the Galactic center at the origin. The $\mathrm{X}$ and $\mathrm{Y}$ axes are in the Galactic plane, and the $\mathrm{Z}$ axis is perpendicular to the plane. The top diagrams in Figure 12 are plots of the densities in the $5^{\circ}$ subdivisions in the range $150^{\circ} \leq \alpha \leq 170^{\circ}$, and they show that the density profile has a pronounced break at $R_{g c} \sim 25 \mathrm{kpc}$ in each subdivision. Combining the data for these 4 subdivisions yields 16 density measurements on each side of the break. Fitting eq 1 separately to these regions, we obtained the following broken power-law profile for the RRLS, and the reduced chi-square, $\chi_{\nu}^{2}$.

$$
\begin{array}{r}
\log (\rho)=\log \left(\rho_{\odot}\right)+n \log (R / 8.0) \\
R=R_{g c} \\
R_{g c}<24.4 \mathrm{kpc} \\
\log \left(\rho_{\odot}\right)=0.80 \pm 0.17, n=-2.8 \pm 0.5, \chi_{\nu}^{2}=0.67 \\
R_{g c}>24.4 \mathrm{kpc}
\end{array}
$$




$$
\log \left(\rho_{\odot}\right)=2.00 \pm 0.31, n=-5.4 \pm 0.5, \chi_{\nu}^{2}=0.54
$$

Top panels in Figure 12 show that this profile is a good fit to the number densities even beyond the distance where the incompleteness of the sample is expected to grow. To estimate the density at the Sun, the profile has to be extrapolated to $8 \mathrm{kpc}$, which yields $\rho_{\odot}=6.2 \pm 3.3 \mathrm{kpc}^{-3}$. If, as suggested above, the completeness of the LSQ survey is $\sim 0.7$, then the true density is $\sim 8.9 \mathrm{kpc}^{-3}$ at the Sun. Multiplying this number by the fraction $\sim 0.74$ of type ab variable in the sample, we obtain $6.6 \mathrm{kpc}^{-3}$ for type ab variables alone, which is higher than some recent estimates (e.g., $4.2 \mathrm{kpc}^{-3}$ Vivas \& Zinn 2006 and 5.6kpc ${ }^{-3}$ Sesar et al. 2010), but within our errors of them.

The middle left panel of the Figure shows that this profile approximately fits the data in the range $170^{\circ} \leq \alpha \leq 180^{\circ}$ before the break, (i.e., $R_{g c}<24.4 \mathrm{kpc}$ ) but it declines somewhat too steeply beyond the break. The same can be said for the next subdivision, $180^{\circ} \leq \alpha \leq 190^{\circ}$, however, this region includes the VSS and the Sgr stream. The bottom panels show that starting in the range $190^{\circ} \leq \alpha \leq 200^{\circ}$ and becoming more pronounced in $200^{\circ} \leq \alpha \leq 210^{\circ}$, the profile runs roughly parallel to the data before the break, but it is offset to higher densities. A systematic deviation from spherical model is expected in this sense if the halo flattened towards the plane. The huge overdensity caused by the Sgr stream in these panels makes any comparison between the profile and the data essentially meaningless beyond the break.

The relatively small range in the directions probed by the survey limit its sensitivity for determining the flattening parameter $\mathrm{q}$, the ratio of the minor axis $(\mathrm{Z})$, to major axes (X and Y) of an ellipsoidal model. We can, however, see if a particular halo model fits the data, and we chose to examine the recent one by Sesar et al. (2011) in which q = 0.7. Following their nomenclature, $R_{e}$ is the semimajor axis of the ellipsoid, and it is substituted for $\mathrm{R}$ in equation 1 . We obtained the following results fitting, as before, the densities in the 
region $150^{\circ} \leq \alpha \leq 170^{\circ}$.

$$
\begin{array}{r}
R=R_{e}=\left(X^{2}+Y^{2}+\left(\frac{Z}{0.7}\right)^{2}\right)^{1 / 2} \\
R_{e}<29.5 \mathrm{kpc} \\
\log \left(\rho_{\odot}\right)=0.85 \pm 0.18, n=-2.5 \pm 0.4, \chi_{\nu}^{2}=0.69 \\
R_{e}>29.5 \mathrm{kpc} \\
\log \left(\rho_{\odot}\right)=2.35 \pm 0.35, n=-5.2 \pm 0.5, \chi_{\nu}^{2}=0.61
\end{array}
$$

The top panels in Figure 13 shows that the fit of this profile to the data in the range $150^{\circ} \leq \alpha \leq 170^{\circ}$, is as good as the one obtained above with $R_{g c}$. There is again clear evidence for a break in the density profile.

The left middle panel shows that this profile is again somewhat too steep beyond the break for the data in the range $170^{\circ} \leq \alpha \leq 180^{\circ}$. The right middle panel shows that in the range $180^{\circ} \leq \alpha \leq 190$, the profile underestimates the density, but this is not surprising because this zone contains the VSS and the Sgr stream. The bottom panels show that in contrast to the spherical profile, this profile fits well the data before the break. The good fit of this profile to the data before the break in all subdivisions save the ones for $180^{\circ} \leq \alpha \leq 190^{\circ}$, where known halo substructures exist, is evidence that the halo is flattened and $q \sim 0.7$. Because of the presence of the Sgr Stream, our data are not suitable for examining whether or not the flattening remains the same beyond the break.

The profile that we find for the ellipsoidal halo is in reasonable agreement with the results of Sesar et al. (2011), who using a sample of main-sequence stars found $q=0.70 \pm 0.01$, a break at $27.8 \pm 0.8 \mathrm{kpc}$, and values of $-2.62 \pm 0.04$ and $-3.8 \pm 0.1$ for the slopes before and after the break, respectively. For the outer slope, our results are in somewhat better agreement with results of Deason et al. (2011), who for a large sample 
of blue horizontal branch stars and blue stragglers found $q \sim 0.6$, a break at $\sim 27 \mathrm{kpc}$, and inner and outer slopes of -2.3 and -4.6. Recent investigations (Watkins et al. 2009; Sesar et al. 2011; Akhter et al. 2012) that have used RRLS as halo tracers have also found breaks in the density profile with slopes similar to what we find here. The break radii found by Watkins et al. (2009) and Sesar et al. (2010) for the RRLS in stripe 82 of the SDSS are similar to what we have found here for a very different direction. Akhter et al. (2012) found a break between 45 and $50 \mathrm{kpc}$ in analysis of the SEKBO RRLS survey. Away from the Sgr stream (i.e. $\alpha<180^{\circ}$ ) where we can examine the halo slope at large distances, we do not find evidence of a break at $45-50 \mathrm{kpc}$ (see Figure 13).

The result of using the ellipsoidal profile to estimate the background RRLS as a function of $\alpha$ and $d_{\odot}$ is shown in Figure 14. The density plus or minus from the profile is given in terms of standard deviations, assuming Poisson statistics and no uncertainty in the profile. The large underdensity at $d_{\odot} \lesssim 5 \mathrm{kpc}$ is caused by the incompleteness due to CCD saturation. At $d_{\odot} \gtrsim 60 \mathrm{kpc}$, the sample is becoming incomplete (see above), which may explain the underdensity seen in most directions at large $d_{\odot}$. The Sgr stream and the Sex dSph galaxy are very prominent overdensities in Figure 14. There is also a large but less extreme overdensity with rough boundaries of $170^{\circ} \lesssim \alpha \lesssim 205^{\circ}$ and $8 \lesssim d_{\odot} \lesssim 20 \mathrm{kpc}$. At the largest $d_{\odot}$, this overdensity coincides with the VSS, and at smaller distances, it may be part of the VOD. It is discussed in more detail below.

\subsection{Comparison with Sgr model by Law \& Majewski}

The Sgr stream is clearly seen in the LSQ data as a large overdensity at distances $>40 \mathrm{kpc}$ and $\alpha \gtrsim 180^{\circ}$. The number of RRLS belonging to the Sgr stream is large enough $(\sim 300)$ that detailed comparisons with models of the disruption of the Sgr galaxy are possible. 
In Figure 15 we show the footprint of the LSQ survey together with the Sgr debris expected in the region according to the recent model by Law \& Maiewski (2010b). The color code of the Sgr debris is the same as in Law \& Majewski (2010b), with the magenta points being particles that became unbound in recent passages of Sgr and cyan and green points corresponding to older debris. We will refer to them as young and old debris respectively, although this has nothing to do with the age of the stellar population they contain but with how long ago they were separated from the main body of Sgr. This figure shows that while there is considerable overlap in the directions to the young and old debris, there are differences, particularly in their distributions with $\alpha$.

To show the differences more clearly, separate plots are made in Figure 16 for the model particles in the young and old streams. Notice that the young and old streams roughly coincide for $\alpha \gtrsim 180$ and $d_{\odot}>30 \mathrm{kpc}$, but the old stream has a longer span in $\alpha$ than the young stream. A simple comparison with Figure 14 reveals a disparity between the model particles for the old stream and the data.

In order to make a better comparison, we need to estimate the normalization factor between the number of particles in the model and the RRLS. To estimate this value, we chose the region encompassing $200^{\circ}<\alpha<210^{\circ}$ and $0^{\circ}<\delta<10^{\circ}$, which has the highest density of Sgr debris. In the range of distances from 40 to $60 \mathrm{kpc}$ there are 546 particles in the model and 131 RRLS. Some of the RRLS may, of course, be halo field stars. We integrated the density profile determined above between 40 and $60 \mathrm{kpc}$, which yielded 8 for expected number of halo RRLS. The remaining excess of 123 RRLS are likely Sgr debris, which gives 4.4 for the ratio between the model particles and RRLS.

The large size of this ratio suggests that LSQ survey contains very few stars that belong to the old debris that crosses the survey region at $\sim 10 \mathrm{kpc}$ (see Figure [16). There are 66 model particles in that nearby stream which means that it should contain $\sim 15$ 
RRLS that are distributed along $\sim 60^{\circ}$ in $\alpha$. That is too low a density to be recognized over the general halo population, which is high at $10 \mathrm{kpc}$.

There is a much better chance of detecting the old debris at greater distances, where the density of the model particles is higher. In Figure [17 we show histograms of the distances of the RRLS and the model particles in 4 bins of $\alpha$. The dotted lines are the sum of the young and old of stream particles divided by 4.4, and the shaded histograms are the young stream alone. The solid lines are the observed numbers of RRLS, after removing the expected number of halo stars. The two upper panels $\left(\alpha>190^{\circ}\right)$ show that the debris is dominated by the young particles. The RRLS distributions match reasonably well both the total number of particles in the young stream and the width of the stream along the line of sight in these two panels. They also follow the predicted gradient in mean distance of the stream in the sense that the debris at $190^{\circ}<\alpha<200^{\circ}$ is closer than at $200^{\circ}<\alpha<210^{\circ}$. There is, however, a systematic difference between the distances of the RRLS and the model. In the uppermost panel the mean distance of the RRLS is $49 \mathrm{kpc}$ while the mean distance of the particles in the model is $51 \mathrm{kpc}$. This small difference is probably due to a difference between the distance scales for the RRLS and for the M giant stars to which the model is tied (see Law \& Majewski 2010b).

Towards decreasing $\alpha$ (lower panels in Figure 17), the old stream becomes more prominent, as the number of young debris particles declines. This produces the differences between the dotted and shadded histograms. Contrary to the model, however, the excess of RRLS diminishes to the point of being almost nonexistent at $170^{\circ}<\alpha<180^{\circ}$. The old stream is predicted by the model to contain 30 RRLS between 40 and $50 \mathrm{kpc}$, which should have been easily detected. But there is essentially no excess of RRLS in this region. The predictions of the Law \& Majewski (2010b)'s model for the young stream are consistent with the distribution of LSQ RRLS, but there is no sign of the older stream, at least in this 
region of the sky.

\subsection{The overdensities in Virgo}

As noted above, Figure 14 reveals a modest overdensity with rough boundaries of $170^{\circ} \lesssim \alpha \lesssim 205^{\circ}$ and $8 \lesssim d_{\odot} \lesssim 20 \mathrm{kpc}$. In this general direction and distance there have been several previous detections of substructure using the spatial distributions and/or the radial velocities of halo tracers (Vivas et al. 2001; Newberg et al. 2002; Duffau et al. 2006; Newberg et al. 2007; Vivas et al. 2008; Jurić et al. 2008; Keller 2010; Walsh et al. 2009; Jerjen et al. 2013). We will focus on the two overdensities that appear to be the best documented, the Virgo Overdensity (VOD) and the Virgo Stellar Stream (VSS), but this may be an over simplification since radial velocity measurements suggest that this region may contain several more substructures (Newberg et al. 2007; Vivas et al. 2008; Brink et al. 2010; Casey et al. 2012; Duffau et al. 2013). Also note that some authors have argued that the VOD and the VSS are different parts of the same overdensity, a conclusion that is hard to rule out at this point.

The largest of the Virgo substructures is the Virgo Overdensity (VOD), which is most clearly recognized as an excess of main-sequence stars in the SDSS survey (Jurić et al. 2008; Bonaca et al. 2012). The recent estimates by Bonaca et al. (2012) indicate it covers $\sim 2000 \mathrm{deg}^{2}$ of the sky and possibly more, has a maximum density at $d_{\odot} \sim 11 \mathrm{kpc}$, and is detected at $d_{\odot}=7 \mathrm{kpc}$. Compared to an axisymmetric region in the southern galactic gap, Bonaca et al. (2012) found an excess of $50 \%$ in the number density of main-sequence stars. Figure 14 indicates that there is modest excess of RRLS at $d_{\odot} \sim 11 \mathrm{kpc}$ from about $\alpha=155^{\circ}\left(1, \mathrm{~b} \sim 240^{\circ}, 50^{\circ}\right)$ to $205^{\circ}\left(1, \mathrm{~b} \sim 330^{\circ}, 65^{\circ}\right)$. According to Figures 2 and 3 in Bonaca et al. (2012), this region is inside the VOD. The region that we have used to estimate the number density of RRLS in the smooth halo (i.e., $\alpha \leq 170^{\circ}$ ) also overlaps with 
the VOD. Consequently, the small excess of RRLS found here may be a consequence of subtracting part of the VOD from itself.

Figure 14 shows that there is a more substantial excess of RRLS at $d_{\odot} \sim 19 \mathrm{kpc}$ in the range $185^{\circ} \leq \alpha \leq 193^{\circ}$, which is the location of the VSS (e.g., Duffau et al. 2006). The distribution in $\alpha$ and $\delta$ of the RRLS in the range $17 \leq d_{\odot} \leq 22 \mathrm{kpc}$ is shown in Figure 18, In this plot, the VSS appears to divide into three concentrations. The two south of $\delta \sim 0$ are similar to the distribution seen in the QUEST RRLS survey (Vivas \& Zinn 2006), and they overlap with the detections of substructure by Newberg et al. (2002), Duffau et al. (2006), Keller et al. (2008), and Keller (2010) using main-sequence turnoff stars, red giants, and subgiants. They also coincide with regions where radial velocity measurements (Duffau et al. 2006; Newberg et al.|2007; Prior et al. 2009; Starkenburg et al. 2009; Brink et al. 2010; Casey et al. 2012; Duffau et al. 2013) have revealed substructure in velocity space. The feature at $\alpha \sim 187^{\circ}$ and $\delta \sim 3^{\circ}$ appears to be the densest of the three concentrations, but by only a small factor that may not be significant. The VSS appears to continue further to the north and may reach $\delta \sim 10^{\circ}$. Figure 18 shows a small excess of RRLS at $\alpha \sim 191^{\circ}$ and $\delta \sim-8^{\circ}$, which coincides with the area where Newberg et al. (2007) found evidence of the VSS in velocity space.

In the same direction as the VOD and the VSS, Walsh et al. (2009) found two small overdensities of stars using photometric data from the SDSS. The most significant of these they dubbed Virgo Z, which they considered to be a candidate UFD galaxy at $d_{\odot} \sim 40$ kpc. Recently, Jerjen et al. (2013) constructed deep color-magnitude diagrams (CMDs) at these positions, $1220-1(\alpha, \delta)=\left(185.077^{\circ},-01.35^{\circ}\right)($ Virgo Z in Walsh et al. 2009) and 1247-00 $\left(191.992^{\circ},-00.75^{\circ}\right)$ and determined that they contain halo substructures at similar distances, $d_{\odot} \sim 23 \mathrm{kpc}$, and that their stellar populations are similar in age, $~ 8.2$ Gyr, and in $[\mathrm{Fe} / \mathrm{H}], \sim-0.7$. Jerien et al. (2013) concluded that these substructures are part of 
the same stellar stream and that it is separate from the Sgr stream, which it resembles in age and $[\mathrm{Fe} / \mathrm{H}]$, but not in $d_{\odot}$. The dominant stellar population of this stream is too young to produce RRLS, but the same is true of the Sgr stream, which is rich in RRLS that are part of its older, more metal-poor population. It is not clear that the stream identified by Jerien et al. (2013) is related to the large concentration of RRLS in this direction, the VSS, because they differ in mean $d_{\odot}$ by $\sim 4 \mathrm{kpc}$.

To see if some LSQ RRLS may be associated with the substructure in 1220-1 and 1247-00, we have plotted in Figure 19 these positions and the LSQ RRLS that are in the interval $19.5 \leq d_{\odot} \leq 27.5 \mathrm{kpc}$. To arrive at this interval, we used the $1 \sigma$ extrema on the distances that Jerien et al. (2013) measured at the two positions and added in quadrature the $7 \%$ uncertainty in the distances of the RRLS that stem from the uncertainty in their absolute magnitudes and from the photometric errors (see Vivas \& Zinn 2006). While this interval may seem large at first, it is important to recall that streams have depth along the line of sight ( $\sim 7 \mathrm{kpc}$ for the Sgr stream, see Figure 17) and that the two positions where this stream has been detected are separated by $\sim 7^{\circ}$ and may in fact have different $d_{\odot}$.

Figure 19 shows that while there is only one RRLS in the above $d_{\odot}$ interval near field 1247-00 (LSQ726 at $\left.d_{\odot}=27.3 \mathrm{kpc}\right)$, there are are several near 1220-1. The 4 RRLS within one degree of the center of 1220-1 are listed in Table 4, where one can see that 3 of the 4 have $d_{\odot} \sim 26.4 \mathrm{kpc}$, which is within $1 \sigma$ of the $d_{\odot}$ of $1220-1(24.3 \pm 2.5 \mathrm{kpc}$, Jerjen et al. 2013). The two stars closest to the center of 1220-1 lie within the field that is plotted in figure 10 of Walsh et al. (2009), and they coincide with regions of high stellar density in that figure. While the 3 stars with similar $d_{\odot}$ in Table 4 appear to be the most likely candidates for membership in this stream, it possible that the it covers much of the area of Figure 19 and many more RRLS may belong.

Figure 18 shows that there are mild over densities at $\alpha \sim 160^{\circ}$ and $\delta \sim+2^{\circ}$ and at 
$\alpha \sim 200^{\circ}$, which may or may not be related to the VSS. The one near $\alpha=200^{\circ}$ coincides with an overdensity in subgiants that was identified by Keller (2010). Radial velocity measurements are needed to test if these features are real and also the possibility that they are related to the VOD, the VSS, or the stream detected by Jerjen et al. (2013).

\section{The Properties of the RR Lyrae Variables}

\subsection{The Oosterhoff Effect}

Much of the huge literature on the properties of RRLS has rightly focussed on the Oosterhoff Effect (Oosterhoff 1939), which can be briefly summarized as follows. The metal-poor $([\mathrm{Fe} / \mathrm{H}]<-1.0) \mathrm{MW}$ globular clusters $(\mathrm{GCs})$ that contain five or more type ab variables divide, with only a few exceptions, into two groups. One group of clusters (Oosterhoff Group I, OoI) have mean periods of the ab variables $\left\langle P_{a b}\right\rangle$ near 0.55 and the other group (OoII) have $\left\langle P_{a b}\right\rangle \sim 0 \mathrm{~d}$ 64. There are also significant differences between the two groups in $\left\langle P_{c}\right\rangle$ and in the ratio of the numbers of type $c$ to the sum of all types $\left(n_{c} /\left(n_{a b}+n_{c}\right)\right)$ (see Table 5, data from Smith 1995). The Oosterhoff dichotomy is produced by the combination of metallicity and horizontal branch morphology (e.g., Castellani et al.

2003; Catelan 2009), and therefore, it is complicated function of the metallicities, ages, and He abundances of the clusters.

The Oosterhoff dichotomy is between the average properties of the RRLS in the GCs. In either the period-amplitude (P-A) diagram (sometimes called the Bailey diagram) or the period histogram there is overlap between the RRLS populating the OoI and OoII clusters. In, for example, the prototypical OoI cluster M3, 13\% of the type ab variables lie along the mean P-A relation of the prototypical OoII cluster M15 (see Cacciari et al. 2005), and a similar fraction of the M15 variables lie along the P-A relation of M3 (e.g., 
Smith 1995; Cacciari et al. 2005; Corwin et al. 2008). In many OoI and OoII clusters, there are RRLS that lie between the OoI and OoII P-A relations (e.g., Cacciari et al. 2005; Székely et al. 2007; Corwin et al. 2008; Zorotovic et al. 2010; Arellano Ferro et al. 2011; Di Criscienzo et al. 2011). Among the MW GCs, only about $10 \%$ have Oosterhoff intermediate (Oo-int) properties, i.e., $0.58 \leq\left\langle P_{a b}\right\rangle \leq 0 \mathrm{~d} 62$, but the frequency of Oo-int GCs is higher in the LMC and in the For and Sgr dSph galaxies (Catelan 2009).

It is not clear that the Oo-int GCs represent a physically distinct class or are simply more even mixtures of OoI and OoII than typical GCs (Smith et al. 2009). Their P-A diagrams do not show clear evidence of a sequence that is intermediate between the ones for the prototypical OoI and OoII clusters M3 and M15 (e.g., Kuehn et al. 2011). The data in Catelan (2009) indicate that $\sim 30 \%$ of the whole sample of Oo-int clusters in the MW and its satellites are $\leq 0$ d 005 from the boundaries in $\left\langle P_{a b}\right\rangle$ of the Oo-int region, which suggests that the noise may have pushed some OoI and OoII clusters into the Oo-int period range. On the other hand, the majority of the Oo-int clusters occupy a small area in a plot of [Fe/H] against HB type (Catelan 2009), which suggests that noise alone is not the whole explanation.

The majority of the dSph galaxies of that are either satellites of the MW or M31 (e.g., Pritzl et al. 2005; Kinemuchi et al. 2008) and one UFD galaxy (Garofalo et al. 2013) are classified Oo-int on the basis of $\left\langle P_{a b}\right\rangle$. Their RRLS populations are typically mixtures of stars that lie on the OoI and OoII sequences and in between (e.g., Siegel \& Majewski 2000; Held et al. 2001; Pritzl et al. 2005; Kinemuchi et al. 2008). This is not unexpected because these systems have metallicity spreads that overlap with the $[\mathrm{Fe} / \mathrm{H}]$ ranges of the OoI and OoII GCs. Since many of these systems have unusually red HB morphologies compared to GCs of the same mean $[\mathrm{Fe} / \mathrm{H}]$, HB morphology may be partially responsible as well. The best example of this may be the Draco dSph galaxy, whose red HB peters out in the 
instability strip (Kinemuchi et al. 2008).

\subsection{The Oosterhoff Effect in subdivisions of the LSQ sample}

The P-A diagram for the LSQ RRLS is shown in the upper left diagram of Figure 20, where one can see that it has the customary form (see Smith 1995). Before plotting this diagram, we removed the stars that are probably members of the Sex dSph galaxy because they are not part of the halo field population. Figure 20 shows there are a small number of the type $\mathrm{c}$ variables that have very low amplitudes $(\Delta V<0.2)$, which raises some concern that they are not bona fide RRLS. Since a few globular clusters (e.g., NGC 2419 Di Criscienzo et al. 2011) and the LMC (Soszyñski et al. 2010) contain RRLS with similar properties, we have kept these stars in our catalog.

Following many previous workers, we compare the LSQ RRLS in the P-A diagram with the mean loci for the type ab variables OoI and OoII clusters (from Cacciari et al. 2005; Zorotovic et al. 2010), but caution that the amplitudes for some stars are probably affected by the Blazhko effect, the cyclic variation in amplitude over periods of typically 20 to 200 days (e.g., Smith 1995). Studies of RRLS over long time base lines have shown that $\geq 40 \%$ the type ab and 5-40\% of the type c variables (e.g., Kolenberg et al. 2010) exhibit the Blazhko effect and vary in amplitude by tenths of magnitude. Since the LSQ observations were obtained over only a few months, they are inadequate for identifying stars that exhibit the Blazhko effect. Consequently, the amplitudes that we obtained may vary anywhere from the minimum to the maximum of the Blazhko cycle, which will produce scatter in the P-A diagram (e.g., Cacciari et al. 2005). The period distributions LSQ RRLS should be unaffected. Since many of the investigations of the RRLS in GCs and the dSph galaxies were also obtained over short periods of time, some of the scatter in their P-A diagrams may also be due to the Blazhko effect. 
The type c RRLS in some OoI and OoII clusters follow different P-A relationships (e.g., Smith 1995; Cacciari et al. 2005; Corwin et al. 2008; Zorotovic et al. 2010), but when classifying by Oosterhoff type, most authors put more weight on the type ab then the type c variables. For example, Zorotovic et al. (2010) classified the GC NGC 5286 as OoII, although nearly all of its type c variables scatter around the P-A relation for OoI. Because they appear to be a less reliable indicator of Oosterhoff type, we do not consider the P-A relationships of the type c variables in the following.

Figure 20 contains the P-A diagrams and the period distributions for subdivisions of the LSQ sample of particular interest (see also Table 5). Every subdivision includes the whole range in $\delta$ of the survey. The one labelled Sgr Stream is for the region defined by $193^{\circ} \leq \alpha \leq 210^{\circ}$ and $37 \leq d_{\odot} \leq 55 \mathrm{kpc}$, which encompasses the densest part of the leading stellar stream from the Sgr dSph galaxy. The next subdivision, $180^{\circ} \leq \alpha \leq 195^{\circ}$ and $15 \leq d_{\odot} \leq 22 \mathrm{kpc}$, is called the VSS Region because it contains the densest part of the overdensity that is identified above with the VSS. Note that unlike the Sgr Stream, the contrast between the VSS and the smooth halo background is not huge and contamination from the background may be significant. The next subdivision is the region, $\alpha \leq 170^{\circ}$, that we have used above to estimate the density profile of the Smooth Halo. The final two subdivisions include the variables of the Smooth Halo inside and outside the break in the density profile at $R_{g c} \sim 25 \mathrm{kpc}$. The region of the break, where there may be overlap if two separate populations of RRLS exist, has been purposely avoided.

For the subdivision of the LSQ variables, $\left\langle P_{a b}\right\rangle$ and $\left\langle P_{c}\right\rangle$, and the standard deviations of the samples, $\sigma P_{a b}$ and $\sigma P_{c}$, and $n_{c} /\left(n_{a b}+n_{c}\right)$ are listed in Table 5 . The quantity OoII $\%$ is defined as follows. In the P-A diagram, we placed a curve of the same shape as the OoI and OoII curves from Zorotovic et al. (2010) midway between the ones for OoI and OoII and then determined the percentage of the total sample of type ab RRLS that lie on the OoII 
side of this curve. This procedure gives an idea of the relative weighting of the OoI and OoII RRLS in the sample. It ignores the possibility that a distinct class of Oo-int RRLS exists, which seems consistent with P-A diagrams of Oo-int GCs. Since the P-A diagram is affected by the Blazkho effect, we caution that OoII \% may not a useful parameter to compare separate studies that have different observational time spans. This should not be a factor here, where we use it to compare different samples of LSQ RRLS. The errors that are listed in Table 5 for $n_{c} /\left(n_{a b}+n_{c}\right)$ and OoII \% are based on Poisson statistics and are therefore lower limits.

In each of the P-A diagrams of Figure 20, the type ab variables scatter around the fiducials for OoI and OoII, with some variables in between. This and the values of $\left\langle P_{a b}\right\rangle$, $\left\langle P_{c}\right\rangle, n_{c} /\left(n_{a b}+n_{c}\right)$, and OoII \% in Table 5 suggest that in each of these regions there is a mixture of OoI and OoII that is weighted more towards OoI than OoII.

On the basis of $\left\langle P_{a b}\right\rangle$, the VSS region is classified as OoI, but it is close to the borderline. The differences between it and the Smooth Halo sample are minor, although the latter is formally Oo-int. The Kolmogorov-Smirnov (K-S) two sample test indicates that there is no reason to reject the hypothesis that the period distributions of the type ab variables in the VSS region and the Smooth Halo have been drawn from the same parent distribution. The value of $\left\langle P_{a b}\right\rangle$ found here for the VSS agrees with the value, $\left\langle P_{a b}\right\rangle=$ 0.57 , that is obtained from 10 RRLS that are radial velocity members (Duffau et al. 2013). Radial velocity measurements are needed for more stars before the Oosterhoff properties of the VSS can be precisely determined.

In the Smooth Halo sample, there are no significant differences between the samples of RRLS on either side of the break in the density profile. This suggests that there is not a large change across the break that affect the properties of RRLS (e.g., metallicity and/or HB morphology). 
The mixture of OoI, OoII, and intermediate objects that we find here for the Smooth Halo is similar to what is seen in the P-A diagrams of other regions of the halo (Kinemuchi et al. 2006; Miceli et al. 2008; Szczygieł et al. 2009), but not all. In the area studied by Kinman et al. (2012), the fraction of OoII variables is smaller than that found here, which suggests that the properties of the Smooth Halo varies with position in the Galaxy (see also Miceli et al. 2008).

\subsection{The MW halo and its massive satellite galaxies}

According to the accretion scenario for the formation of the MW halo, the RRLS in the outer halo should resemble the ones found in dwarf galaxies. Since the majority of the dSph satellite galaxies of both the MW and M31 are Oo-int, several authors (e.g., Catelan

2009; Clementini 2010) have concluded that the MW halo was unlikely to have formed from protogalactic fragments that resembled the present-day dSph galaxies. While the Smooth Halo sample discussed above is formally Oo-int (see Table 4), its very near the borderline with OoI, and it contains a larger fraction of OoI variables than most $\mathrm{dSph}$ galaxies. Because theoretical simulations of satellite accretion suggest that much of the accreted halo comes from the tidal destruction of a few massive satellites (e.g., Bullock \& Johnston 2005; Zolotov et al. 2009), it is of interest to compare the RRLS populations of the MW halo and massive dwarf galaxies to see if they provide a better match than the dSph galaxies in general. Here we compare the halo to the LMC and the SMC, and the two most luminous dSph galaxies, Sgr and For, which are the only MW satellites that contain GCs. The mere presence of GCs in the outer halo suggests that the accretion, if it is the origin of the halo, included at least a few galaxies that were sufficiently massive to form their own GCs. The accretion of a massive satellite, similar to the LMC, may be the origin of the metal-rich tail on the metallicity distribution of the halo (Frebel 2010). Other dwarf 
galaxies in the Local Group are known to contain both GCs and RRLS, and they too may be analogues of the massive satellites that have been accreted. Unfortunately, the existing surveys of their RRLS populations are in small fields, typically at the outskirts of the galaxies, and therefore, they may not be representative of the whole galaxies (e.g., M32, see Sarajedini et al. 2012). For the comparisons that we make here, it is important to note that the estimates of the total population of RRLS in For range from $\sim 660$ (Vivas \& Zinn 2006), which is based on the sample of Bersier \& Wood (2002) and consequently may be too low (see Greco et al. 2005), to $\sim 2000$ (Clementini 2010), and that there are several thousands of RRLS already measured in each of the other galaxies. The accretion of just one galaxy that resembled any one of these four would put as many, and possibly several times more RRLS in the halo than all the other known dSph and UFD galaxies combined (see Clementini 2010). Each of the 4 galaxies has experienced long histories of star and cluster formation, which in the cases of the LMC and the SMC continue to the present time. Since we are considering here only the RRLS, which are very old, these galaxies may be used as analogues of galaxies that merged with the MW a few billion years after the Big Bang, a time when much of the accreted halo formed according to the theoretical simulations (Bullock \& Johnston 2005; Zolotov et al. 2009). We have ignored the putative dwarf galaxy in Canis Major in this discussion because it is not certain that it even exists and because if it does, it contains very few RRLS (Mateu et al. 2009).

In Table 5, we have collected data for the LMC and the SMC from the OGLE project (Soszyński et al. 2009; Soszyñski et al. 2010). Because we are unable to separate type d and type e RRLS from type c in the LSQ survey, the results in Table 5 were obtained by lumping together these types for the LMC and SMC. The sample of Sgr RRLS is from Cseresnjes (2001), and to limit the possible contamination from RRLS in the galactic bulge or halo, we limited this sample to RRLS in the more centrally located of his two fields and to ones fainter than $B_{i}=18.6$ (see Cseresnjes et al. 2000). The type d variables in this 
sample are counted as type c here. The For data are from Bersier \& Wood (2002) who did not use the P-A diagram to decide pulsation type, but instead divided the stars into type c and type ab depending on whether their periods were to the short or long period side of 0.47 day. Bersier and Wood noted that they rejected many possible RRLS in order to obtain a clean sample of RRLS instead of a more complete one. Since Greco et al. (2005) found similar results for $\left\langle P_{a b}\right\rangle$ from another, although smaller, sample of For RRLS, the mean properties of the Bersier and Wood sample are probably representative of the galaxy.

On the basis of the values of $\left\langle P_{a b}\right\rangle$ in Table 5, the LMC and Sgr are OoI and the SMC and For are Oo-int. Note, however, if we had adopted the results that the MACHO project had obtained for the LMC (Alcock et al. 1996), it would be classed as Oo-int, albeit near the OoI borderline, which underscores that different samples, although large in numbers of variables, can lead to different classifications for the same object. In terms of $\left\langle P_{a b}\right\rangle$, every galaxy in Table 5 lies near the boundary between OoI and Oo-int. The values of $\left\langle P_{c}\right\rangle$ and $n_{c} /\left(n_{a b}+n_{c}\right)$ are consistent with OoI or Oo-int, but not OoII. The period distributions of the type ab variables in these galaxies are compared with the one for the Smooth Halo in Figure 21, which reveals some interesting similarities and differences. As noted by Cseresnjes (2001), the period distributions of the LMC and Sgr are strikingly similar. Large differences exist between the distributions of the LMC and the SMC (Soszyñski et al. 2010), particularly at the shorter periods. Compared to the other galaxies, For has a particularly narrow distribution, but one wonders if this may be due in part to the incompleteness of the sample (see Bersier \& Wood 2002; Greco et al. 2005). We selected only the Smooth Halo sample to compare these distributions, because the other regions of the LSQ survey contain contributions from either the Sgr Stream or the VSS. Figure 21] shows that the Smooth Halo sample has a broad period distribution that resembles more the distributions of Sgr and the LMC than the ones of the other two galaxies. While there is no reason to believe that the Smooth Halo originated from the accretion of just one galaxy, on statistical 
grounds (i.e., the K-S test at 0.05 significance) there is no reason to reject the hypothesis that the Smooth Halo has the same period distributions as the LMC or Sgr.

The P-A diagram for Sgr (see fig. 8 in Cseresnjes 2001) shows that a large fraction of its type ab variables scatter around the OoI relation. The P-A diagrams of the LMC and the SMC samples are shown in Figure 22. Because the OGLE project measured the amplitudes in the Cousin's I-band (Soszyński et al.|2009), it was necessary to transform the V-band P-A relationships for OoI and OoII (Zorotovic et al. 2010) to the I-band. To do this, we used equation 1 in Dorfi \& Feuchtinger (1999), which is based on the V-and I-band amplitudes of RRLS in several GCs. Figure 22 shows that in both the LMC and the SMC, there are large concentrations of type ab variables to left and slightly to the right of the OoI curve, and relatively few variables in the vicinity of the OoII curve. In the LMC diagram, there is a dense band of type ab stars, which at large amplitudes lies to the right of the OoI curve, but at lower amplitudes coincides with the curve or to the left of it. It is not clear if this is a sign that the curve, which is based on many fewer RRLS in the prototypical OoI GC M3 (Cacciari et al. 2005), does not accurately trace the OoI sequence, or if the LMC has an strong Oo-int component that manifests itself at only the largest amplitudes (i.e., $\Delta I \geq 0.6 \mathrm{mag})$. In either case, it is clear that the accretion of galaxies similar to the LMC or the SMC would have heavily weighted the halo toward OoI. Because Bersier \& Wood (2002) did not measure the amplitudes of the RRLS, it is not possible to plot a P-A diagram for the For sample. It is unlikely to contain a large population of OoII variables because only $2 \%$ of its type ab variables have $\mathrm{P} \geq 0 \mathrm{~d} 68$ day, whereas OoII GCs typically have larger percentages (e.g., 26\% in the case of the OoII GC NGC 2419, Di Criscienzo et al. 2011). The P-A diagram that Greco et al. (2005) plotted for their sample of 110 RRLS in For clearly shows that the majority of the type ab variables follow the OoI relation.

The accretion of galaxies similar to the ones in Table 5 may explain the preponderance 
of OoI variables in the MW halo. The accretion of galaxies similar to the other dSph galaxies, mostly Oo-int, and the UFDs, mostly OoII, is also expected if the accretion hypothesis is correct. In the period distribution and the P-A diagram, the RRLS from these other sources will largely blend in with ones from any massive satellites, and the relative contributions of these different types of systems may be difficult to disentangle. Because the existing high mass satellites contain small fractions of stars near the OoII sequence, the low-mass satellites may be more important source for OoII than for OoI stars.

If the galaxies in Table 5 are truly analogues of the galaxies that were accreted, then their GC populations should also resemble that of the outer halo. Mackey \& Gilmore (2004); van den Bergh \& Mackey (2004); Mackey \& van den Bergh (2005) have compared the GCs in these galaxies with the ones in the outer halo of the MW, and found substantial overlap in terms of position in the HB type - $[\mathrm{Fe} / \mathrm{H}]$ plane, luminosity, and structural parameters such as half-light radius and core radius. They also noted that in terms of these parameters the outer halo GCs are distinctly different from the inner halo and bulge/disk GCs. They concluded that the GCs of the outer MW halo are likely to have been accreted from dwarf galaxies resembling the LMC, SMC, and the Sgr and For dSph galaxies. Catelan (2009) has argued, however, that the GCs in the LMC, For and Sgr are not a good match to the ones in the MW because several of them lie in a triangular area of the HB type - $[\mathrm{Fe} / \mathrm{H}]$ plane that is unoccupied by MW GCs. This small region appears to be largely responsible for the Oo-int phenomenon, and therefore it may be the origin of the larger frequencies of Oo-int GCs in these dwarf galaxies than in the MW halo (Catelan 2009). To occupy this region a $\mathrm{GC}$ must have $[\mathrm{Fe} / \mathrm{H}]$ and age within narrow ranges (see fig. 7 Catelan 2009); consequently, it is unclear that all dwarf galaxies must necessarily contain such clusters and have greater frequencies of Oo-int clusters than the outer halo of the MW. A good example of the vagaries of GC formation in dwarf galaxies is provided by the SMC. Despite its large population of very old stars, e.g., the RRLS, the SMC contains just one old GC, NGC 
121 (e.g., Piatti et al. 2007), which contains too few RRLS to be classified by Oosterhoff type. In terms of position in the HB type - $[\mathrm{Fe} / \mathrm{H}]$ plane, luminosity, and structural parameters, it is similar to the GCs in the outer halo of the MW (Mackey \& Gilmore 2004; van den Bergh \& Mackey 2004; Mackey \& van den Bergh 2005), but it does not lie in the triangular area associated with the Oo-int phenomenon.

The hypothesis that the accretion of satellite galaxies was a major contributor to the MW halo does not require that an exact match exist between the halo and the still existing satellites, just that the properties of the halo be consistent with a physically reasonable mixture of the old stellar populations and the GC populations of extant satellites in general (e.g., Zinn 1993; Mackey \& Gilmore 2004). The papers by Mackey, van den Bergh and Gilmore (see above) suggest that this is the case for the outer halo GCs. The comparisons that we have made here suggest that the RRLS population of the outer halo may be explained by satellite accretion, if it included some relatively massive systems.

\subsection{The RRLS populations of the Sgr Stream and its main body}

One case where one might expect an exact match of RRLS populations is between the Sgr Stream and its main body, and this appears not to be the case. The data in Table 5 show that a difference exists between them in $\left\langle P_{a b}\right\rangle$. We have examined this further by using the K-S two sample test to see if their distributions in $P_{a b}$ are indeed different. With the LSQ sample for the Sgr Stream and the sample described above from Cseresnjes (2001) for the main body, the K-S test indicates that the null hypothesis that they were drawn from the same parent distribution can be rejected at a significance level $<0.025$. The estimated probability density functions of the two samples are compared in Figure 23, where one can see that the major difference is that the main-body sample has a higher percentage $(\sim 20 \%)$ of variables with $P_{a b} \leq 0.52$ than the stream $(\sim 10 \%)$. Because Cseresnjes (2001) did not 
find a significant gradient in RRLS properties across the main-body, this difference appears to be limited to one between the outskirts of the pre-disturbed galaxy, which presumably now make up the Sgr streams, and its central regions.

Among type ab RRLS, there is a rough, inverse correlation between period and $[\mathrm{Fe} / \mathrm{H}]$. According to the relationship that Sarajedini et al. (2006) determined from the comparison of the periods and spectroscopically determined $[\mathrm{Fe} / \mathrm{H}]$ values for 132 field type ab variables, the period of 0.52 day corresponds to $[\mathrm{Fe} / \mathrm{H}] \sim-1.2$. The difference between the period distributions in Figure 23 may be a sign that the $[\mathrm{Fe} / \mathrm{H}]$ distribution of the main-body has a more extensive metal-rich tail, $\lesssim-1.2$, than does the stream. Since other studies have found evidence for chemical abundance differences along the Sgr streams and/or between the main-body and the streams (Chou et al. 2007; Monaco et al. 2007; Chou et al. 2010; Shi et al. 2012), this explanation seems most likely, although alternatives, such as a difference in distribution of stars accross the instability strip cannot be ruled out.

\section{Summary}

Our survey of $\sim 840 \operatorname{deg}^{2}$ of the sky has yielded light curves, periods, and mean magnitudes for 1013 type ab and 359 type c RRLS. The completeness of the survey is estimated to be $\gtrsim 70 \%$ over the range $8 \lesssim d_{\odot} \lesssim 60 \mathrm{kpc}$ and decline to zero at $d_{\odot} \sim 100$ kpc. The survey region overlaps with part of the Sex dSph galaxy, and 6 new RRLS and 3 new $\mathrm{AC}$ variables were discovered in this galaxy.

In directions away from the prominent halo substructures, the spatial distribution of the LSQ RRLS is consistent with an ellipsoidal model that is flattened towards the galactic plane. A ratio of minor to major axes of 0.7, as estimated by Sesar et al. (2011), provides a good match to our RRLS data. The number density profile of the RRLS has a break in its 
power-law slope at $R_{g c} \sim 25 \mathrm{kpc}$, in the sense that it is clearly steeper at larger distances. Since other investigators have seen similar breaks in different directions, at approximately the same $R_{g c}$, and using a variety of halo tracers (RRLS, main-sequence stars and blue horizontal branch stars), this break appears to be a general feature of the MW halo. We do not find evidence for a change in $\left\langle P_{a b}\right\rangle,\left\langle P_{c}\right\rangle, n_{c} /\left(n_{a b}+n_{c}\right)$, or OoII \% across the break. It is important to examine this again once larger samples of RRLS are available.

The most prominent substructure in the survey region is the leading stream from the Sgr dSph galaxy, which contains $\sim 20 \%$ of the whole sample of RRLS. In the direction and distance from the Sun, the stream outlined by the RRLS is consistent with the predictions of the recent model by Law \& Majewski (2010b) for stars that were stripped from the galaxy between 1.3 and 3.2 Gyr ago. According to this model, stars that were stripped from the galaxy between 3.2 and 5.0 Gyrs have a broader distribution in $\alpha$ than the more recently stripped stars. Our observations do not agree with this prediction for the older stream stars. Our failure to detect this older stream may mean that it contains far fewer stars than predicted by the model, is displaced out our survey region, or is coincident in direction and distance with the younger stream.

The Sgr stream has a lower percentage of short period type ab variables than the main-body of the galaxy. One possible interpretation is that main-body of Sgr has a more extensive metal-rich tail to its $[\mathrm{Fe} / \mathrm{H}]$ distribution than the stream. This is consistent with other evidence that suggests that metallicity differences exist between the main-body of Sgr and its streams.

The second most prominent over density is related to the previously identified substructures in Virgo, the VOD and the VSS. The densest part of this feature coincides in direction and distance, $\sim 20 \mathrm{kpc}$ with the VSS. Our survey provides evidence that the VSS extends to the north of previous detections, i.e., $\delta \gtrsim 2^{\circ}$. In our data, the VSS is the 
most distant part of a substructure that extends over the range $8 \lesssim d_{\odot} \lesssim 20 \mathrm{kpc}$, which on the near side overlaps with the much larger VOD (Jurić et al. 2008; Bonaca et al. 2012, see). We caution that this apparent connection in space between the VSS and VOD may not mean a common origin. Radial velocity measurements (see Duffau et al. 2013) reveal that there are several different clusterings in velocity and distance that are more indicative of several separate moving groups rather than one large one. It is not clear at this time, if any of these groups are related to the substructure that Jerjen et al. (2013) have recently discovered in the CMDs of the fields 1220-1 and 1247-00, which is in the same direction but appears to be more distant at $d_{\odot} \sim 23 \mathrm{kpc}$. Some LSQ RRLS that lie close to these positions have values of $d_{\odot}$, which suggest possible membership in this new stellar stream.

The RRLS in the Sgr leading stream, the VSS, and the smooth halo are similar to each other in $\left\langle P_{a b}\right\rangle,\left\langle P_{c}\right\rangle, n_{c} /\left(n_{a b}+n_{c}\right)$, and OoII \%. Their values of $\left\langle P_{a b}\right\rangle$ place them near the dividing line between $\mathrm{OoI}$ and Oo-int. In the P-A diagram, their type ab variables resemble a mixture of OoI and OoII that is more heavily weighted towards OoI $(\sim 27 \%$ OoII $)$. These samples of RRLS in the MW halo resemble the ones found in the field populations of the 4 most massive satellite galaxies of the MW. The similarities between the MW halo and these satellite galaxies are consistent with theoretical results, which suggest that the accretion of a few massive satellite galaxies produces a large fraction of a galaxy's halo.

We thank the anonymous referee whose comments on the manuscript have led to several improvements.

This research has been supported by NSF grant AST-1108948 and DOE grant DE FG0 ER92 40704 to Yale University and by the Provost Office of Yale University. This project would not have been possible without the public release of the data from the Sloan Digital Sky Survey III. Funding for SDSS-III has been provided by the Alfred P. Sloan Foundation, the Participating Institutions, the National Science Foundation, and the U.S. Department 
of Energy Office of Science. The SDSS-III web site is http://www.sdss3.org/.

SDSS-III is managed by the Astrophysical Research Consortium for the Participating Institutions of the SDSS-III Collaboration including the University of Arizona, the Brazilian Participation Group, Brookhaven National Laboratory, University of Cambridge, Carnegie Mellon University, University of Florida, the French Participation Group, the German Participation Group, Harvard University, the Instituto de Astrofisica de Canarias, the Michigan State/Notre Dame/JINA Participation Group, Johns Hopkins University, Lawrence Berkeley National Laboratory, Max Planck Institute for Astrophysics, Max Planck Institute for Extraterrestrial Physics, New Mexico State University, New York University, Ohio State University, Pennsylvania State University, University of Portsmouth, Princeton University, the Spanish Participation Group, University of Tokyo, University of Utah, Vanderbilt University, University of Virginia, University of Washington, and Yale University. 


\section{REFERENCES}

Akhter, S., Da Costa, G. S., Keller, S. C., \& Schmidt, B. P. 2012, ApJ, 756, 23

Alcock, C., Allsman, R. A., Axelrod, T. S., et al. 1996, AJ, 111, 1146

Arellano Ferro, A., Figuera Jaimes, R., Giridhar, S., et al. 2011, MNRAS, 416, 2265

Baltay, C., Rabinowitz, D., Hadjiyska, E., et al. 2013, PASP, 125, 683

Beers, T. C., Carollo, D., Ivezić, Ž., et al. 2012, ApJ, 746, 34

Bell, E. F., Zucker D. B., Belokurov, V., et al. 2008, ApJ, 680, 295

Belokurov, V. Evans, N. W., Bell, E. F., et al. 2007a, ApJ, 657, L89

Belokurov, V., Evans, N. W., Irwin, M. J., et al. 2007b, ApJ, 658, 337

Belokurov, V., Zucker, D. B., Evans, N. W., et al. 2006, ApJ, 647, L111

Bersier, D., \& Wood, P. R. 2002, AJ, 123, 840

Bertin, E., \& Arnouts, S. 1996, A\&AS, 117, 393

Bessell, M. S. 1990, PASP, 102, 1181

Bonaca, A., Jurić, M., Ivezić, Ž. et al. 2012, AJ, 143, 105

Brink, T. G., Mateo, M., \& Martínez-Delgado, D. 2010, AJ, 140, 1337

Bullock, J. S., \& Johnston, K. V. 2005, ApJ, 635, 931

Cacciari, C., Corwin, T. M., \& Carney, B. W. 2005, AJ, 129, 267

Carney, B. W., Latham, D. W., \& Laird, J. B. 1990, AJ, 99, 572

Carollo, D., Beers, T. C., Chiba, M., et al. 2010, ApJ, 712, 692 
Carollo, D., Beers, T. C., Lee Y. S., et al. 2007, Nature, 450, 1020

Carraro, G., Zinn, R., \& Moni Bidin, C. 2007, A\&A, 466, 181

Casey, A. R., Keller, S. C., \& Da Costa, G. 2012, AJ, 143, 88

Castellani, M., Caputo, F., \& Castellani, V. 2003, A\&A, 410, 871

Catelan, M. 2009, Ap\&SS, 320, 261

Chou, M.-Y., Cunha, K., Majewski, S. R., et al. 2010, ApJ, 708, 1290

Chou, M.-Y., Majewski, S. R., Cunha, K. et al. 2007, ApJ, 670, 346

Clementini, G. 2010, in Variable Stars, the Galactic halo and Galaxy Formation, ed. C. Sterken, N. Samus, \& L. Szabados, 107

Clewley, L., \& Kinman, T. D. 2006, MNRAS, 371, L11

Corwin, T. M., Borissova, J., Stetson, P. B., et al. 2008, AJ, 135, 1459

Cseresnjes, P. 2001, A\&A, 375, 909

Cseresnjes, P., Alard, C., \& Guibert, J. 2000, A\&A, 357, 871

Da Costa, G. S., \& Armandroff, T. E. 1995, AJ, 109, 2533

Deason, A. J., Belokurov, V., \& Evans, N. W. 2011, MNRAS, 416, 2903

Di Criscienzo, M., Greco, C., Ripepi, V., et al. 2011, AJ, 141, 81

Dorfi, E. A., \& Feuchtinger, M. U. 1999, A\&A, 348, 815

Dotter, A., Sarajedini, A., \& Anderson, J. 2011, ApJ, 738, 74

Drake, A. J., Catelan, M., Djorgovski, S. G., et al. 2013a, ApJ, 763, 32 
-. 2013b, ApJ, 765, 154

Duffau, S., Vivas, A. K., Zinn, R., Ruiz, M. T., \& Méndez, R. A. 2013, A\&A, submitted

Duffau, S., Zinn, R., Vivas, A. K., et al. 2006, ApJ, 636, L97

Eggen, O. J., Lynden-Bell, D., \& Sandage, A. R. 1962, ApJ, 136, 748

Font, A. S., McCarthy, I. G., Crain, R. A., et al. 2011, MNRAS, 416, 2802

Forbes, D. A., \& Bridges, T. 2010, MNRAS, 404, 1203

Frebel, A. 2010, Astronomische Nachrichten, 331, 474

Frebel, A., \& Norris, J. E. 2013, in Planets, Stars and Stellar Systems. Volume 5: Galactic Structure and Stellar Populations, ed. T. D. Oswalt \& G. Gilmore (New York: Springer), 55

Garofalo, A., Cusano, F., Clementini, G., et al. 2013, ApJ, 767, 62

Geisler, D., Wallerstein, G., Smith, V. V., \& Casetti-Dinescu, D. I. 2007, PASP, 119, 939

Greco, C., Clementini, G., Held, E. V. et al. 2005, ArXiv Astrophysics e-prints

Grillmair, C. J. 2006, ApJ, 645, L37

Hadjiyska, E., Rabinowitz, D., Baltay, C., et al. 2012, in IAU Symposium, Vol. 285, IAU Symposium, ed. E. Griffin, R. Hanisch, \& R. Seaman, 324-326

Hattori, K., Yoshii, Y., Beers, T. C., Carollo, D., \& Lee, Y. S. 2013, ApJ, 763, L17

Held, E. V., Clementini, G., Rizzi, L., et al. 2001, ApJ, 562, L39

Helmi, A., Cooper, A. P., White, S. D. M., et al. 2011, ApJ, 733, L7

Helmi, A., White, S. D. M., de Zeeuw, P. T., \& Zhao, H. 1999, Nature, 402, 53 
Ibata, R., Irwin, M., Lewis, G., Ferguson, A. M. N., \& Tanvir, N. 2001a, Nature, 412, 49

Ibata, R., Lewis, G. F., Irwin, M., Totten, E., \& Quinn, T. 2001b, ApJ, 551, 294

Irwin, M., \& Hatzidimitriou, D. 1995, MNRAS, 277, 1354

Ivezić, Ž., Goldston, J., Finlator, K., et al. 2000, AJ, 120, 963

Ivezić, Ž., Vivas, A. K., Lupton, R. H., \& Zinn, R. 2005, AJ, 129, 1096

Jerjen, H., Da Costa, G. S., Willman, B. et al. 2013, ApJ, 769, 14

Jurić, M., Ivezić, Ž., Brooks, A. et al. 2008, ApJ, 673, 864

Keller, S. C. 2010, PASA, 27, 45

Keller, S. C., da Costa, G. S., \& Prior, S. L. 2009, MNRAS, 394, 1045

Keller, S. C., Mackey, D., \& Da Costa, G. S. 2012, ApJ, 744, 57

Keller, S. C., Murphy, S., Prior, S., Da Costa, G., \& Schmidt, B. 2008, ApJ, 678, 851

Kinemuchi, K., Harris, H. C., Smith, H. A., et al. 2008, AJ, 136, 1921

Kinemuchi, K., Smith, H. A., Woźniak, P. R. et al. 2006, AJ, 132, 1202

Kinman, T. D., Cacciari, C., Bragaglia, A., Smart, R., \& Spagna, A. 2012, MNRAS, 422, 2116

Kinman, T. D., Wirtanen, C. A., \& Janes, K. A. 1966, ApJS, 13, 379

Kolenberg, K. Szabó, R., Kurtz, D. W., et al. 2010, ApJ, 713, L198

Kollmeier, J. A., Szczygieł, D. M., Burns, C. R., et al. 2013, ApJ, 775, 57

Kuehn, C. A., Smith, H. A., Catelan, M., Pritzl, B. J., De Lee, N., \& Borissova, J. 2011, AJ, 142, 107 
Lafler, J., \& Kinman, T. D. 1965, ApJS, 11, 216

Law, D. R., \& Majewski, S. R. 2010a, ApJ, 718, 1128

-. 2010b, ApJ, 714, 229

Layden, A. C. 1998, AJ, 115, 193

Lee, Y.-W., Gim, H. B., \& Casetti-Dinescu, D. I. 2007, ApJ, 661, L49

Lupton, R. 2005, http://www.sdss.org/dr5/algorithms/sdssUBVRItransform.html

Mackey, A. D., \& Gilmore, G. F. 2004, MNRAS, 355, 504

Mackey, A. D., Huxor, A. P., Ferguson, A. M. N., et al. 2010, ApJ, 717, L11

Mackey, A. D., \& van den Bergh, S. 2005, MNRAS, 360, 631

Majaess, D. 2010, Acta Astron., 60, 55

Martínez-Delgado, D., Pohlen, M., Gabany, R. J., et al. 2009, ApJ, 692, 955

Mateo, M., Fischer, P., \& Krzeminski, W. 1995, AJ, 110, 2166

Mateo, M. L. 1998, ARA\&A, 36, 435

Mateu, C., Vivas, A. K., Zinn, R., Miller, L. R., \& Abad, C. 2009, AJ, 137, 4412

Miceli, A., Rest, A., Stubbs, C. W., et al. 2008, ApJ, 678, 865

Monaco, L., Bellazzini, M., Bonifacio, P., et al. 2007, A\&A, 464, 201

Morrison, H. L., Helmi, A., Sun, J., et al. 2009, ApJ, 694, 130

Newberg, H. J., Yanny, B., Cole, N., et al. 2007, ApJ, 668, 221

Newberg, H. J., Yanny, B., Rockosi, C., et al. 2002, ApJ, 569, 245 
Nissen, P. E., \& Schuster, W. J. 2010, A\&A, 511, L10

-. 2011, A\&A, 530, A15

Norris, J., Bessell, M. S., \& Pickles, A. J. 1985, ApJS, 58, 463

Oosterhoff, P. T. 1939, The Observatory, 62, 104

Pawlowski, M. S., Pflamm-Altenburg, J., \& Kroupa, P. 2012, MNRAS, 423, 1109

Piatti, A. E., Sarajedini, A., Geisler, D., Gallart, C., \& Wischnjewsky, M. 2007, MNRAS, 381, L84

Preston, G. W., Shectman, S. A., \& Beers, T. C. 1991, ApJ, 375, 121

Prior, S. L., Da Costa, G. S., Keller, S. C., \& Murphy, S. J. 2009, ApJ, 691, 306

Pritzl, B. J., Armandroff, T. E., Jacoby, G. H., \& Da Costa, G. S. 2005, AJ, 129, 2232

Roederer, I. U. 2009, AJ, 137, 272

Saha, A. 1985, ApJ, 289, 310

Saha, A., \& Hoessel, J. G. 1990, AJ, 99, 97

Sarajedini, A., Barker, M. K., Geisler, D., Harding, P., \& Schommer, R. 2006, AJ, 132, 1361

Sarajedini, A., Yang, S.-C., Monachesi, A., Lauer, T. R., \& Trager, S. C. 2012, MNRAS, 425,1459

Schlaufman, K. C., Rockosi, C. M., Lee, Y. S et al. 2009, ApJ, 703, 2177

Schlegel, D. J., Finkbeiner, D. P., \& Davis, M. 1998, ApJ, 500, 525

Schönrich, R., Asplund, M., \& Casagrande, L. 2011, MNRAS, 415, 3807 
Schuster, W. J., Moreno, E., Nissen, P. E., \& Pichardo, B. 2012, A\&A, 538, A21

Searle, L., \& Zinn, R. 1978, ApJ, 225, 357

Sesar, B., Cohen, J. G., Levitan, D., et al. 2012, ApJ, 755, 134

Sesar, B., Ivezić, Ž., Grammer, S. H., et al. 2010, ApJ, 708, 717

Sesar, B., Jurić, M., \& Ivezić, Ž., et al. 2011, ApJ, 731, 4

Shang, Z., Zheng, Z., Brinks, E., et al. 1998, ApJ, 504, L23

Sheffield, A. A., Majewski, S. R., Johnston, K. V., et al. 2012, ApJ, 761, 161

Shetrone, M. D., Côté, P., \& Sargent, W. L. W. 2001, ApJ, 548, 592

Shi, W. B., Chen, Y. Q., Carrell, K., \& Zhao, G. 2012, ApJ, 751, 130

Siegel, M. H., \& Majewski, S. R. 2000, AJ, 120, 284

Smith, H. A. 1995, RR Lyrae stars (Cambridge Astrophysics Series, Cambridge, New York: Cambridge University Press, —c1995)

Smith, H. A., Catelan, M., \& Clementini, G. 2009, in American Institute of Physics Conference Series, Vol. 1170, American Institute of Physics Conference Series, ed. J. A. Guzik \& P. A. Bradley, 179-187

Soszyñski, I., Udalski, A., Szymañski, M. K., et al. 2010, Acta Astron., 60, 165

Soszyński, I., Udalski, A., Szymański, M. K., et al. 2009, Acta Astron., 59, 1

Starkenburg, E., Helmi, A., Morrison, H. L., et al. 2009, ApJ, 698, 567

Stetson, P. B. 2000, PASP, 112, 925 
Szczygieł, D. M., Pojmański, G., \& Pilecki, B. 2009, in American Institute of Physics Conference Series, Vol. 1170, American Institute of Physics Conference Series, ed. J. A. Guzik \& P. A. Bradley, 255-257

Székely, P., Kiss, L. L., Jackson, R., et al. 2007, A\&A, 463, 589

Tolstoy, E., Hill, V., \& Tosi, M. 2009, ARA\&A, 47, 371

van den Bergh, S., \& Mackey, A. D. 2004, MNRAS, 354, 713

Vivas, A. K., Jaffé, Y. L., Zinn, R., et al. 2008, AJ, 136, 1645

Vivas, A. K., \& Zinn, R. 2006, AJ, 132, 714

Vivas, A. K., Zinn, R., Abad, C., et al. 2004, AJ, 127, 1158

Vivas, A. K., Zinn, R., Andrews, P., et al. 2001, ApJ, 554, L33

Vivas, A. K., Zinn, R., Subero, Y., \& Hernández, J. 2006, in Revista Mexicana de Astronomia y Astrofisica Conference Series, Vol. 26, 60-63

Walsh, S. M., Willman, B., \& Jerjen, H. 2009, AJ, 137, 450

Watkins, L. L., Evans, N. W., Belokurov, V. et al. 2009, MNRAS, 398, 1757

Willman, B., Blanton, M. R., West, A. A., et al. 2005, AJ, 129, 2692

Wu, C., Qiu, Y. L., Deng, J. S., Hu, J. Y., \& Zhao, Y. H. 2005, AJ, 130, 1640

Xue, X.-X., Rix, H.-W., Yanny, B., et al. 2011, ApJ, 738, 79

Zinn, R. 1993, in Astronomical Society of the Pacific Conference Series, Vol. 48, The Globular Cluster-Galaxy Connection, ed. G. H. Smith \& J. P. Brodie, 38-+

Zolotov, A., Willman, B., Brooks, A. M., et al. 2009, ApJ, 702, 1058 
Zorotovic, M., Catelan, M., Smith, H. A., et al. 2010, AJ, 139, 357

Zucker, D. B., Belokurov, V., Evans, N. W., et al. 2006, ApJ, 643, L103

Zucker, D. B., Kniazev, A. Y., Bell, E. F., et al. 2004, ApJ, 612, L117

This manuscript was prepared with the AAS LATEX macros v5.2. 


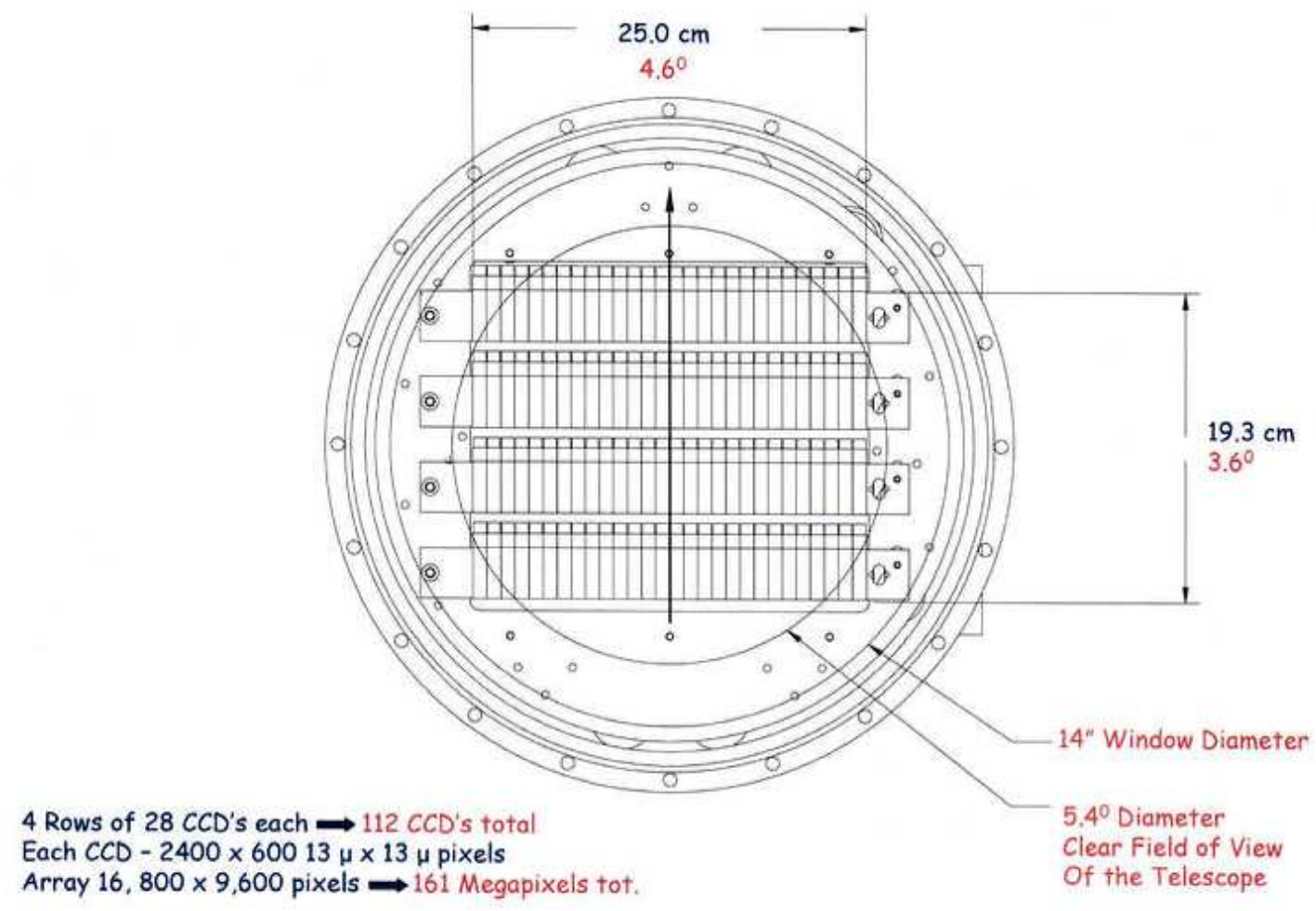

Fig. 1.- The layout of the La Silla QUEST CCD camera. Each of the 4 horizontal rows contains 28 CCD's, which are depicted as narrow rectangles. 


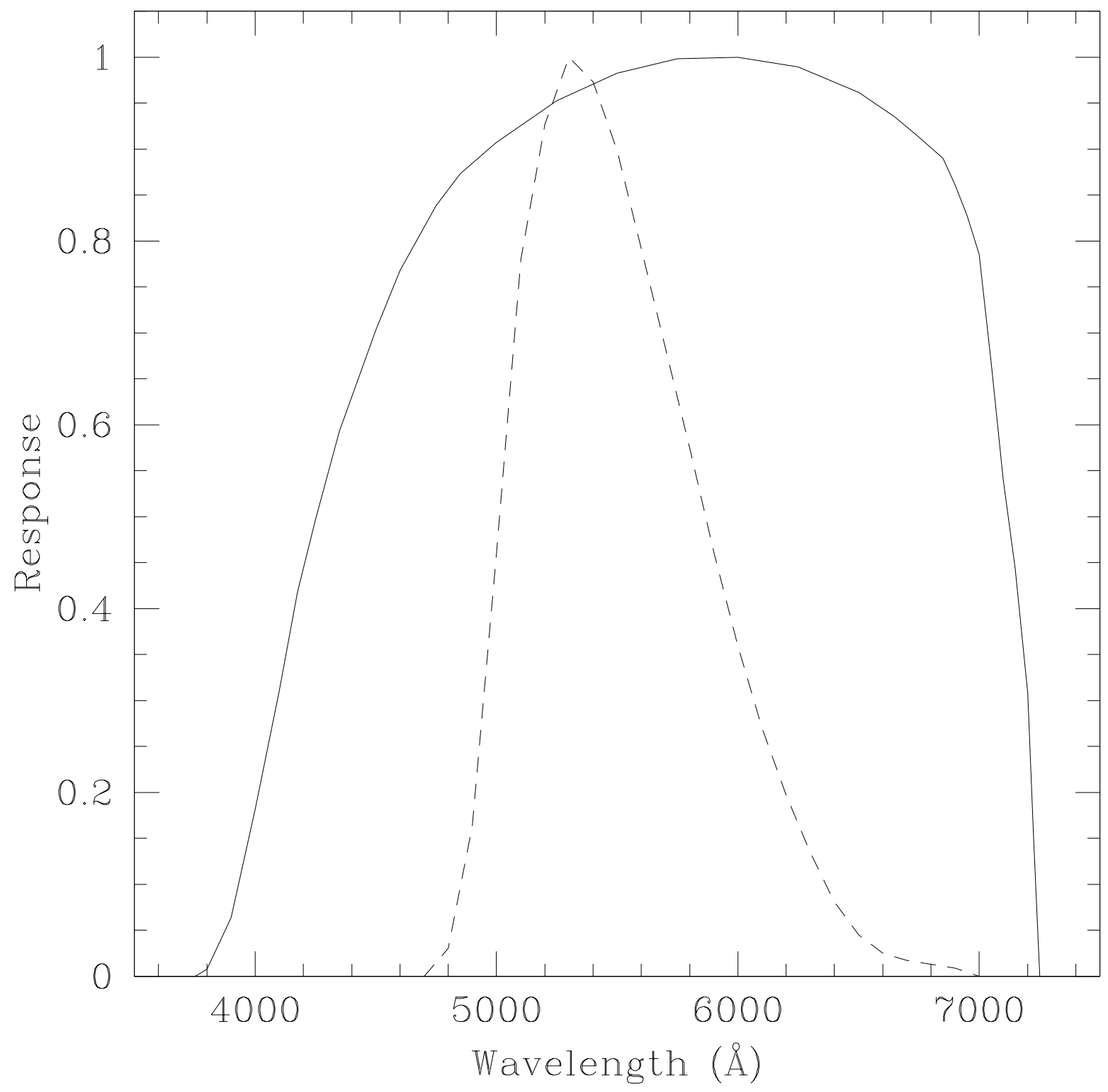

Fig. 2. - The solid curve is the response function of the filter plus CCD used in the LSQ RRLS survey. The dashed curve is the response function of the $\mathrm{V}$ passband as determined by Bessell (1990). 


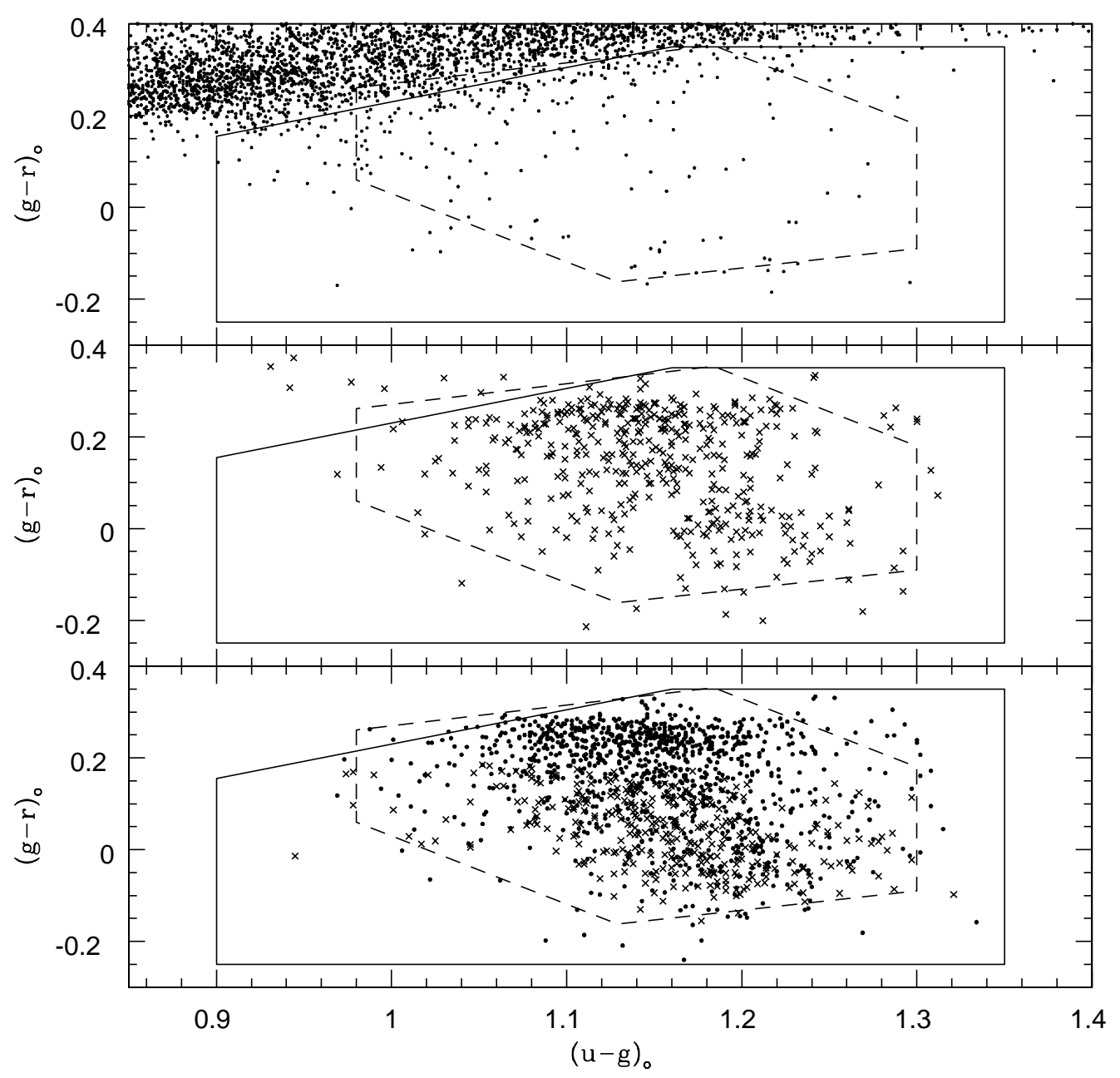

Fig. 3.- The locations of stars in the $(u-g)_{0},(g-r)_{0}$ plane and the selection of RRLS candidates. The solid lines enclose the area in color space that we used to identify RRLS candidates. The dashed lines enclose the area that (Ivezić et al. 2005) used. The top diagram plots the stars in a small area $\left(9 \mathrm{deg}^{2}\right)$ of the LSQ RRLS survey. The large number of stars across the top of this diagram are main-sequence stars. In the middle diagram, the RRLS identified by the QUEST survey (Vivas et al. 2004) are plotted. In the bottom diagram, the type ab (solid circles) and type c (x's) RRLS that are identified in this investigation (Table 1) are plotted. 


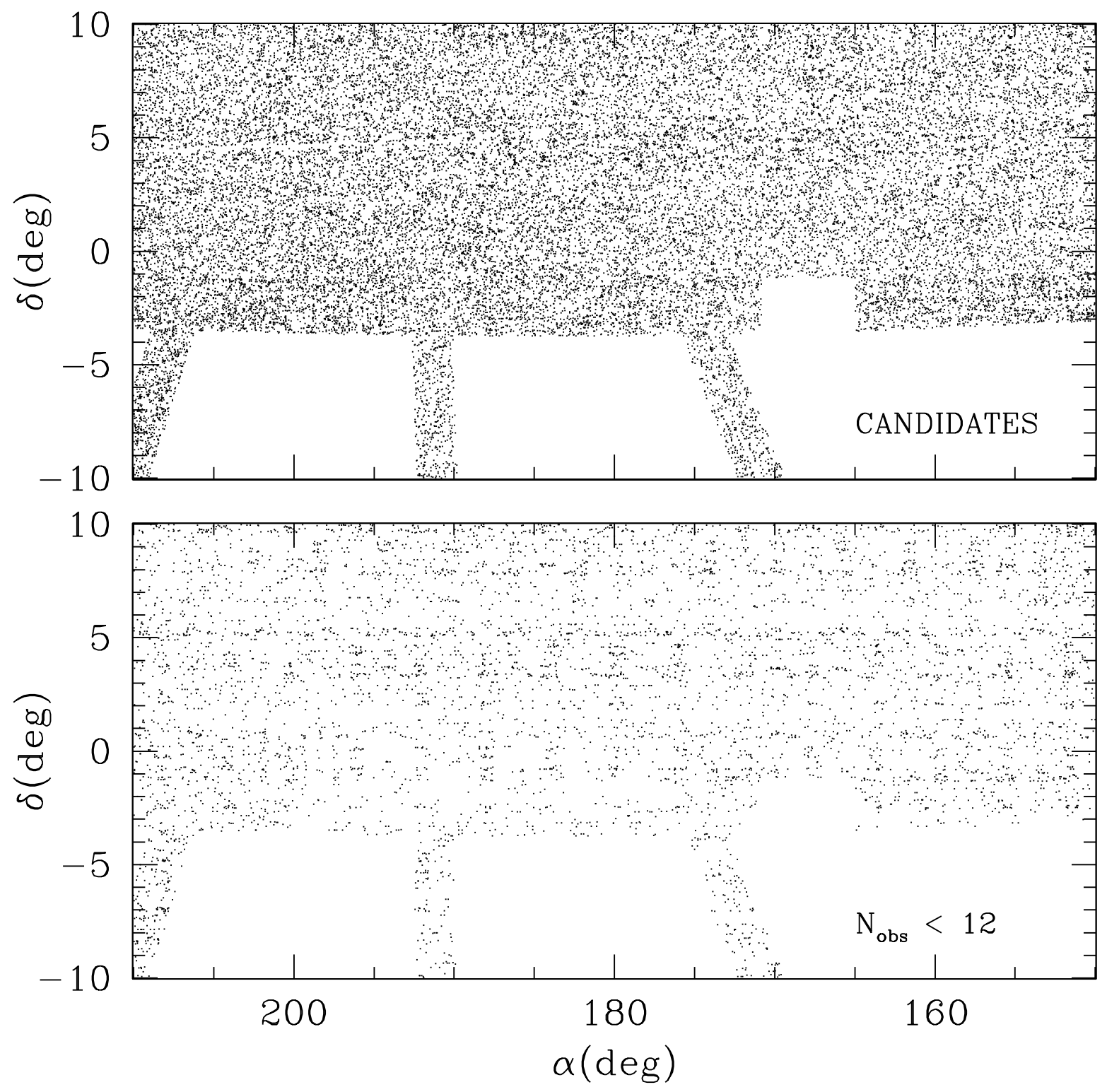

Fig. 4.- The ranges $\alpha$ and $\delta$ included in our RRLS survey. In the top diagram, all of the candidate RRLS are plotted. The shape of the survey region was dictated by the footprint of the SDSS in this area of the sky. In the bottom diagram, the candidate RRLS for which fewer than 12 observations were obtained are plotted. These stars, which for the purpose of identifying RRLS were "unobserved", constitue $20 \%$ of all candidates. 


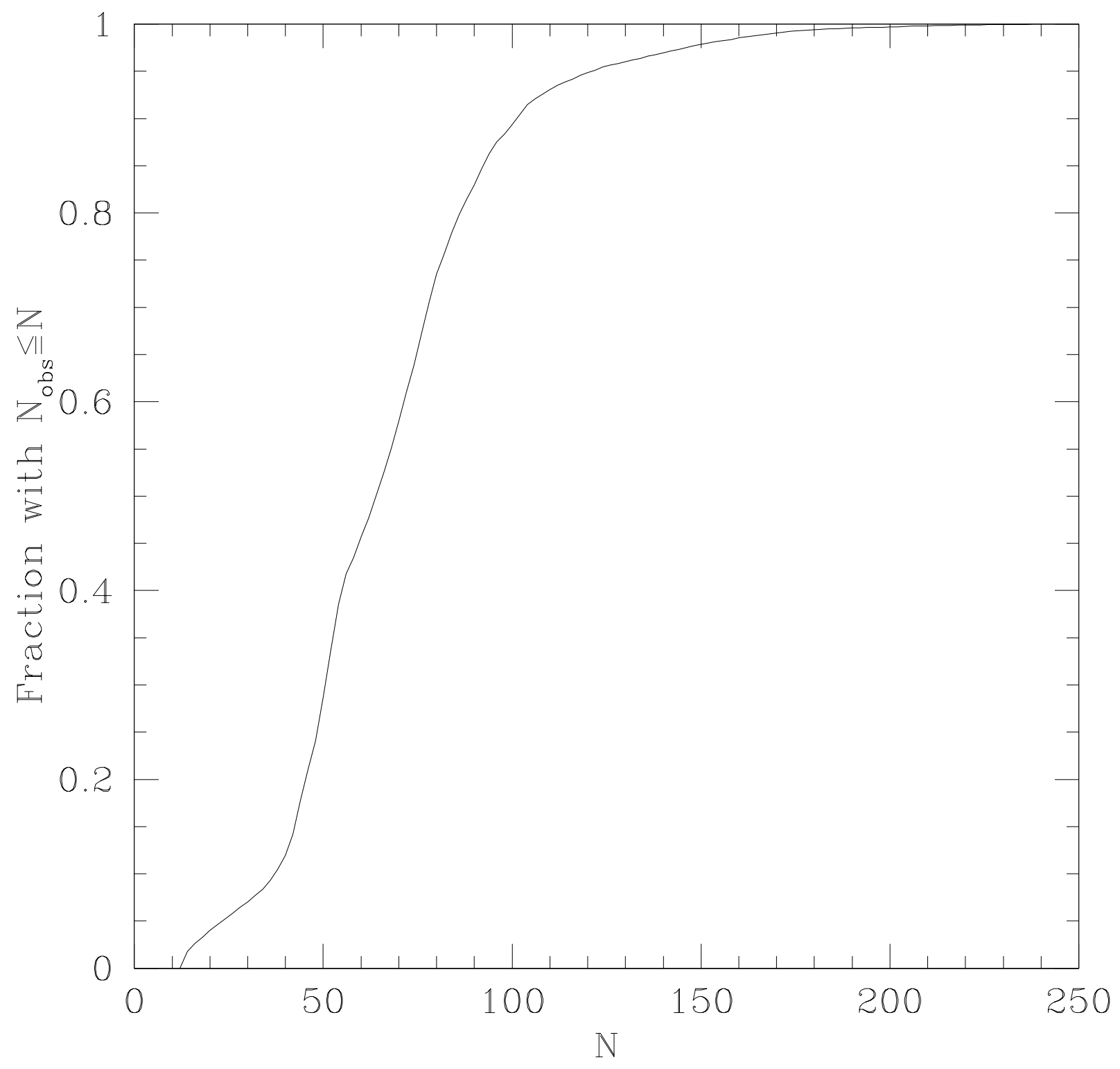

Fig. 5.- The fraction of the sample of "observed" candidates (i.e., $N_{o b s} \geq 12$ ) that have less than $\mathrm{N}$ number of observations is plotted against $\mathrm{N}$. More than $50 \%$ of the sample has $N_{o b s} \geq 60$. 


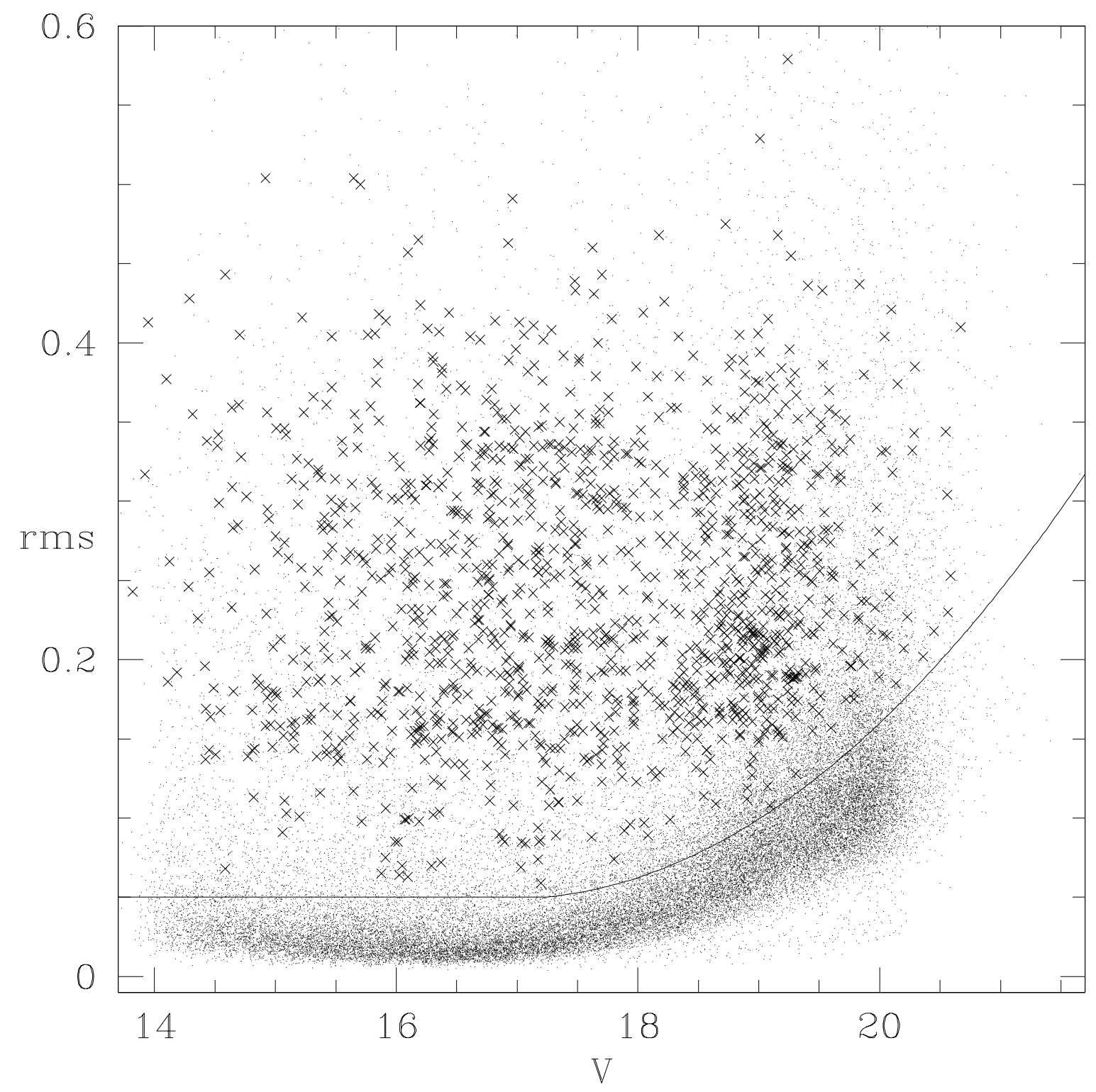

Fig. 6. - The variation of rms with $\langle V\rangle$ for the "observed" candidates (dots). The solid line is the cut made on rms to separate variable from non-variable stars. The x's are the stars identified as RRLS or anomalous Cepheids (Tables 1 \& 2). 


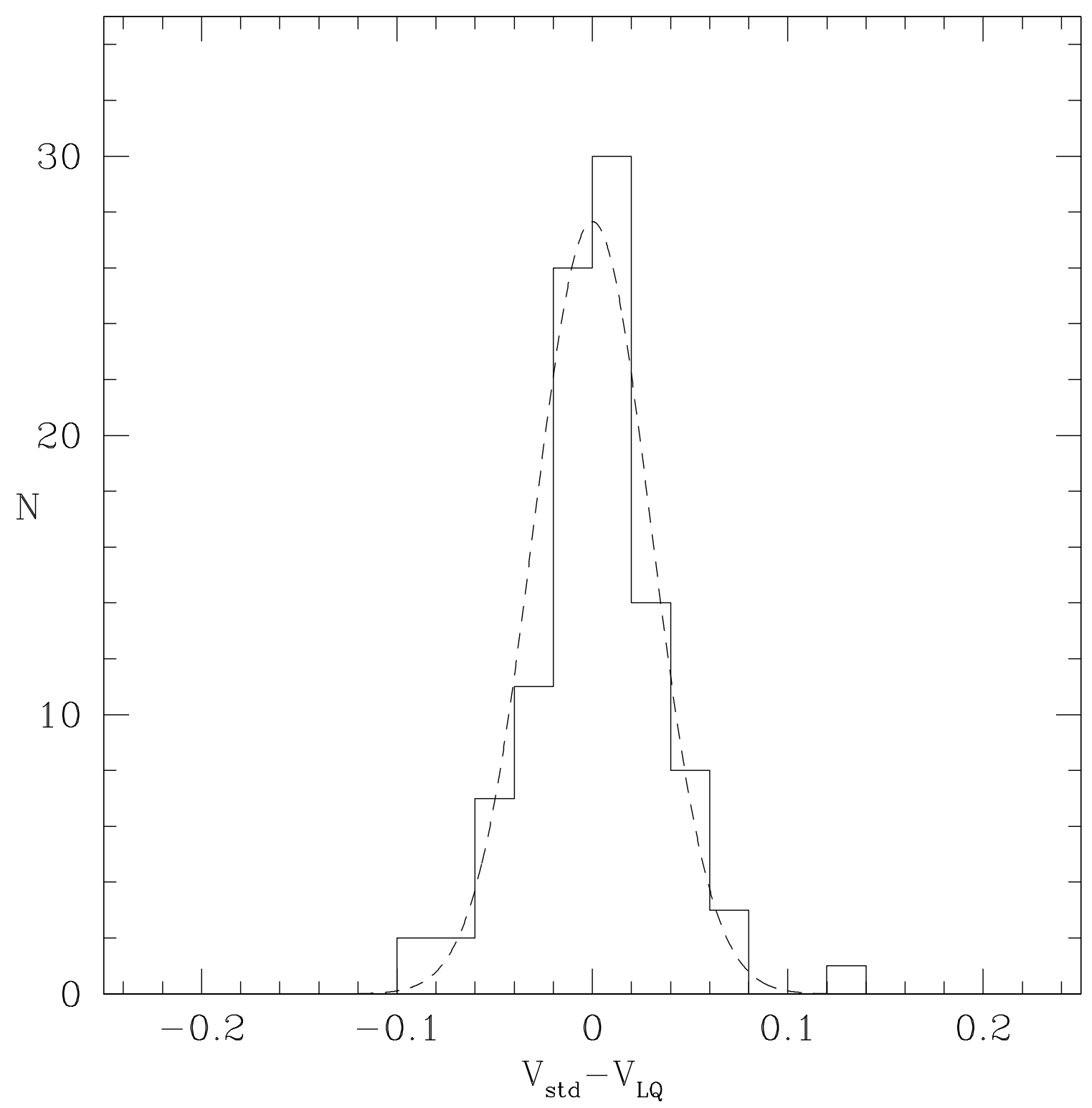

Fig. 7.- For 104 standard stars (Stetson 2000), the histogram of the differences between the standard V and the magnitude obtained here. The dashed curve is a Gaussian distribution with a mean of 0 and $\sigma=0.03$. 


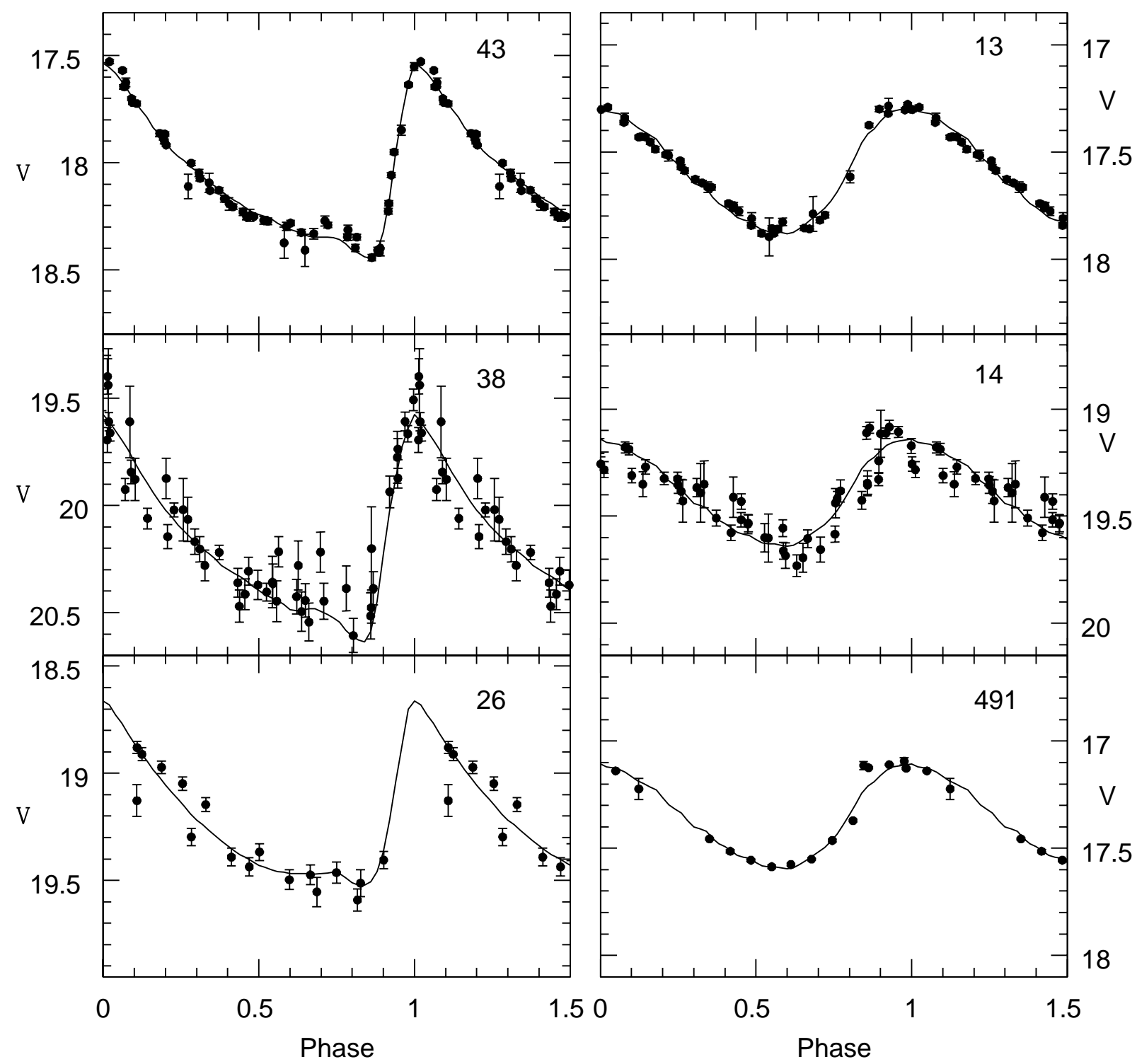

Fig. 8.- Some typical light curves for type ab (left panels) and type c (right panels) produced by the LSQ data. The top panels show stars of roughly average brightness and average number of observations. The middle panels show the light curves of stars near the faint limit of the survey. The bottom panels show stars that have relatively few observations. 


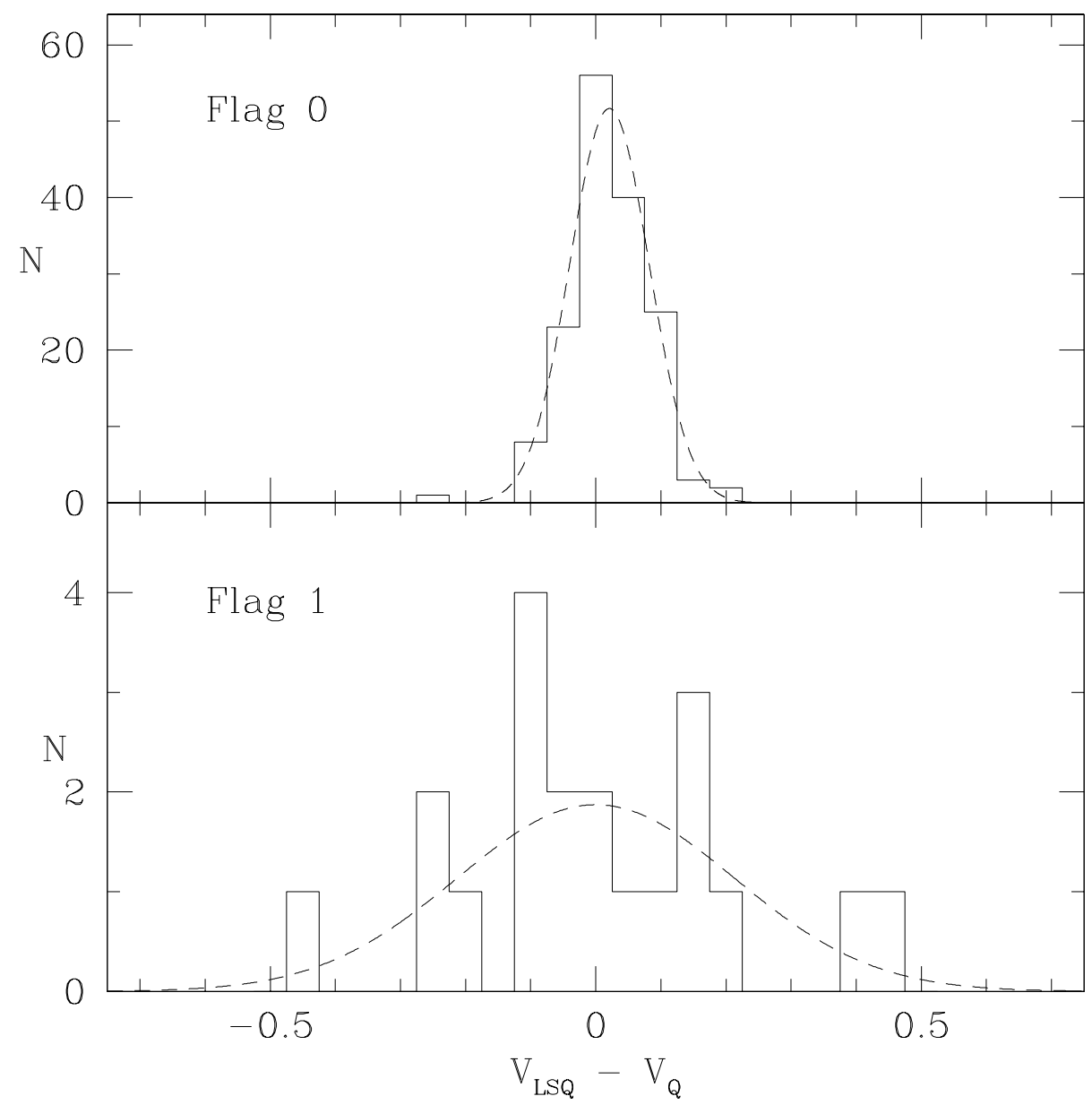

Fig. 9.- The histograms of the differences in $\langle V\rangle$ between the QUEST survey (Vivas et al. 2004) and our survey. The top diagram compares the 158 RRLS in the QUEST survey for which we obtained a good calibration (Flag $=0$ ) of the LSQ data. The histogram is compared with a Gaussian distribution with the same mean (0.021) and $\sigma(0.061)$ as the data. In the bottom diagram, a similar comparison is made for 20 QUEST RRLS for which LSQ calibration is poor (Flag $=1)$. The Gaussian has a mean of 0.006 and $\sigma=0.21$. 


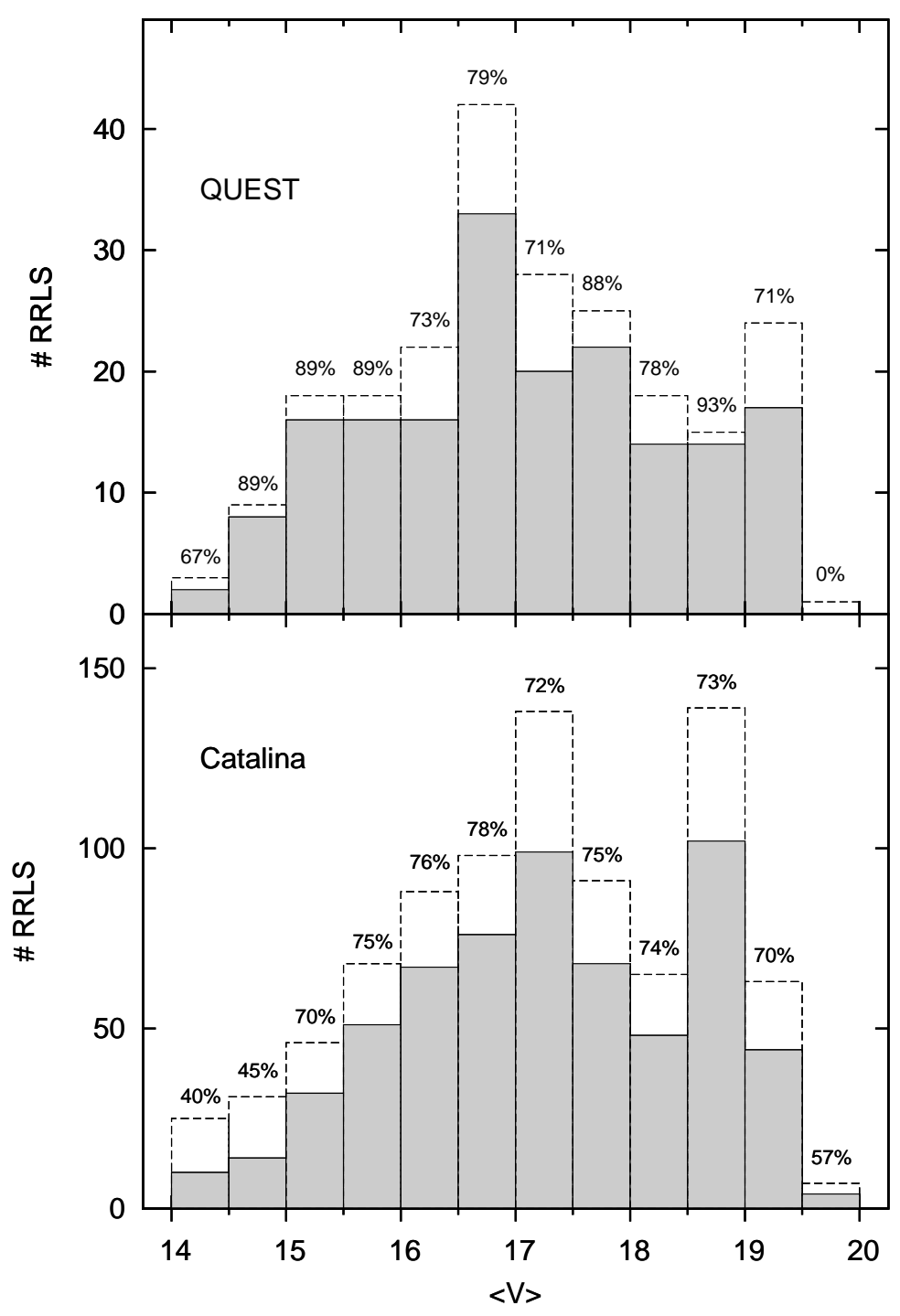

Fig. 10.- In the top and bottom diagrams, the dotted histograms are the distributions with $\langle V\rangle$ of the QUEST and Catalina RRLS in the regions of overlap with the LSQ survey. The filled histrograms is the distribution of the ones recovered by the LSQ survey. The percentages recovered are shown for each bin. 


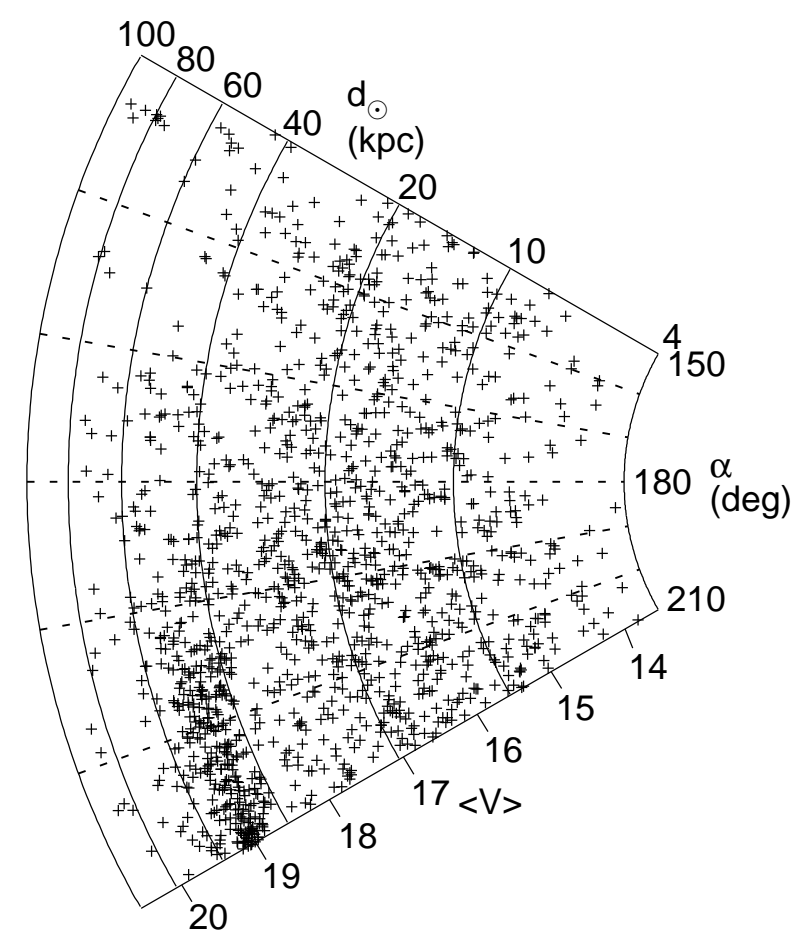

Fig. 11. - For the LSQ RRLS, mean V mag $(\langle V\rangle)$, corrected for extinction, and the corresponding $d_{\odot}$ is plotted against $\alpha$. The large concentration of RRLS from $185^{\circ} \lesssim \alpha \lesssim 210^{\circ}$ with $\langle V\rangle \sim 19$ is the leading stream from the Sgr dSph galaxy. The small concentration of RRLS at $\alpha \sim 153$ and $\langle V\rangle \sim 20$ is the Sex dSph galaxy. The Virgo Stellar Stream (VSS) is located near $\alpha \sim 186^{\circ}$ and $d_{\odot} \sim 20$ (Duffau et al. 2006), where there is a concentration of RRLS in this diagram. 


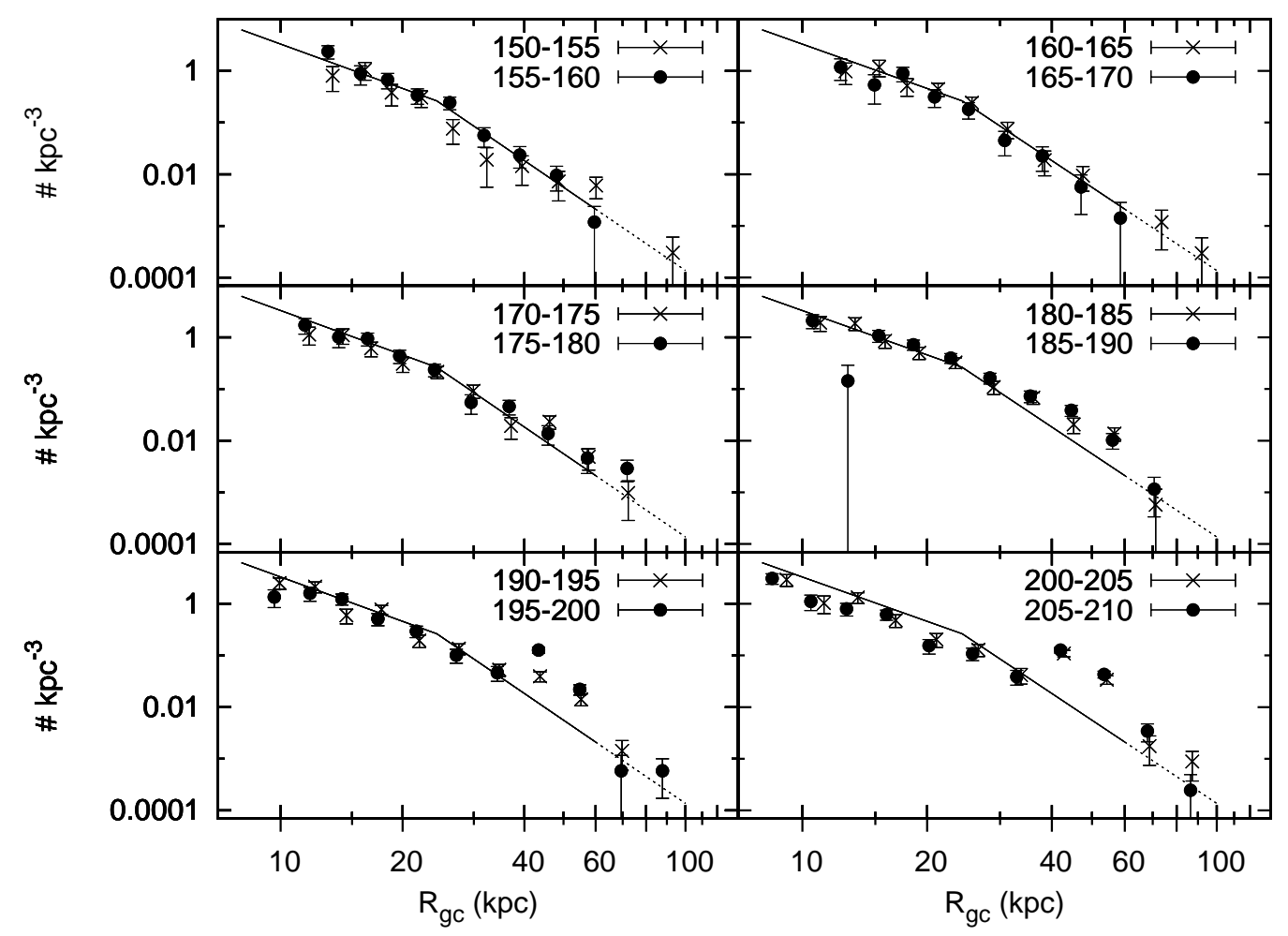




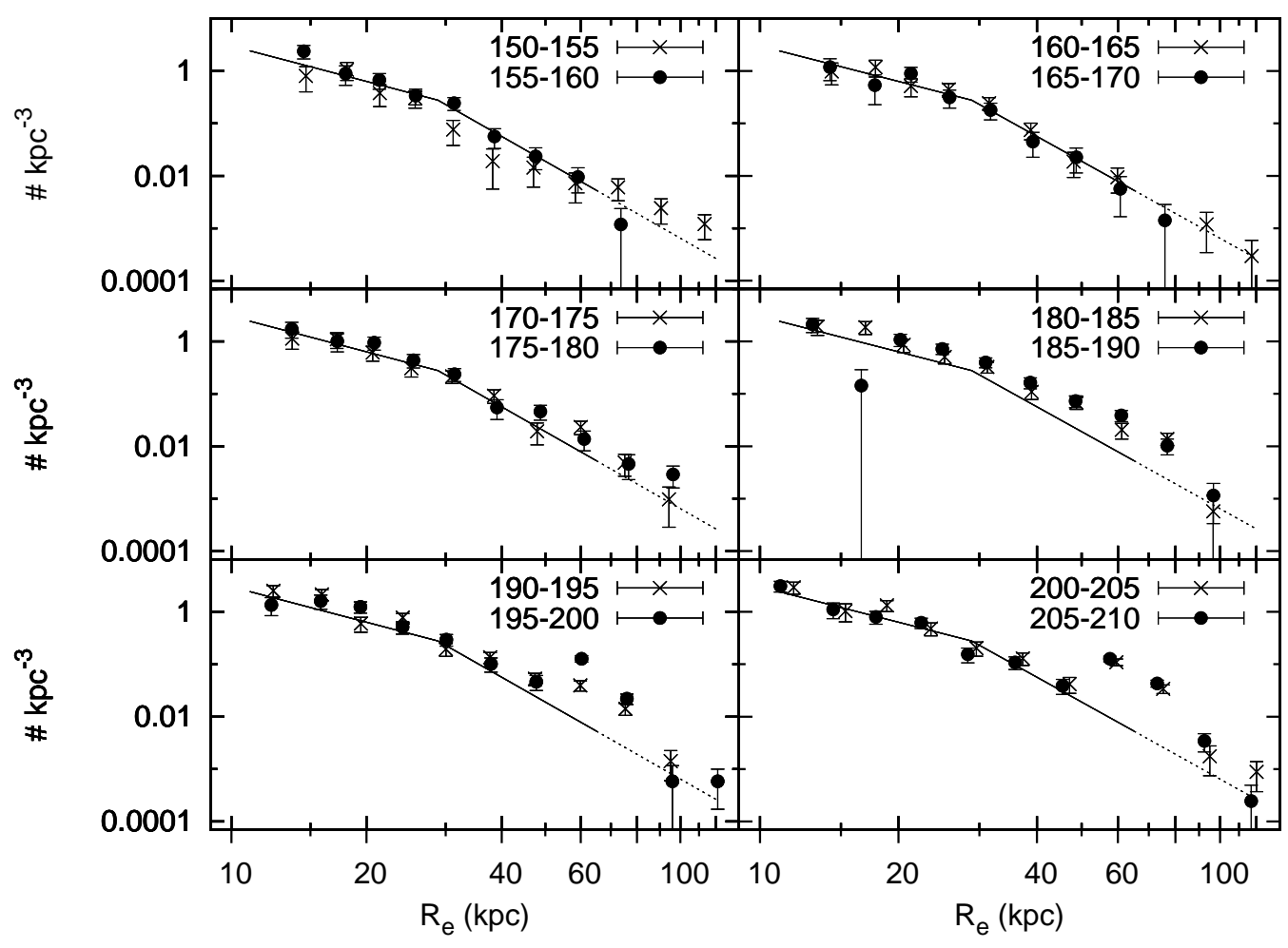




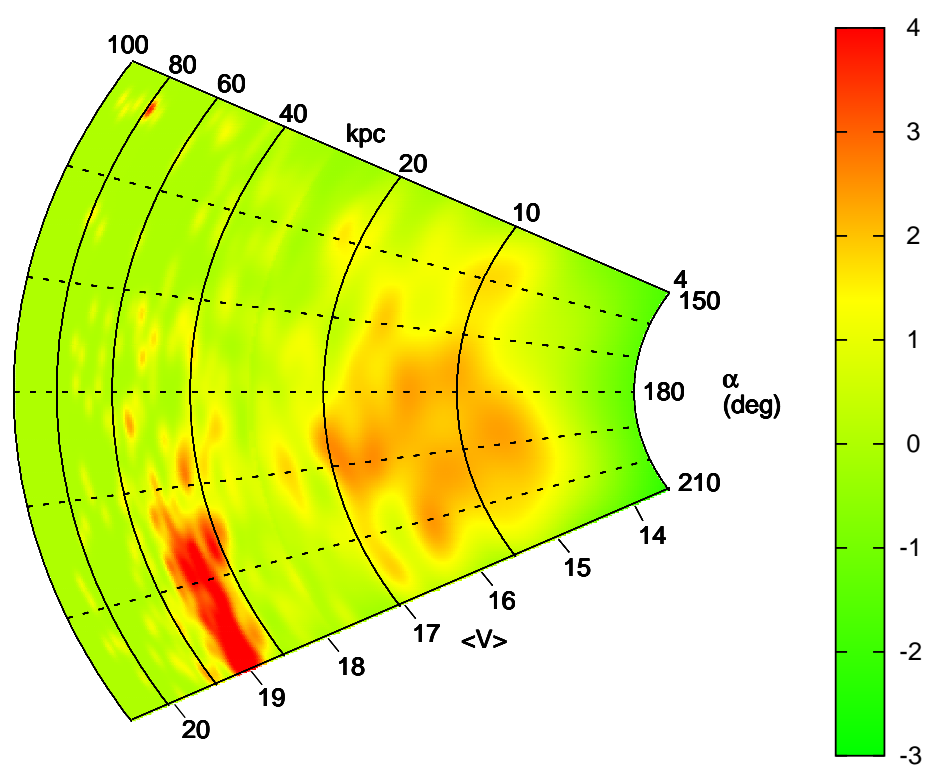

Fig. 14.- The density distribution of the LSQ RRLS as a function of $\alpha$ and $d_{\odot}$. The density scale is in units of standard deviations from the number density profile for the smooth halo (i.e. $\alpha \leq 170^{\circ}$ ). The highest densities in the Sgr Stream are greater than 4 std. dev. above the background (see Figure 13), and therefore saturate on the density scale of this figure. See the electronic edition of the Journal for a color version of this figure. 


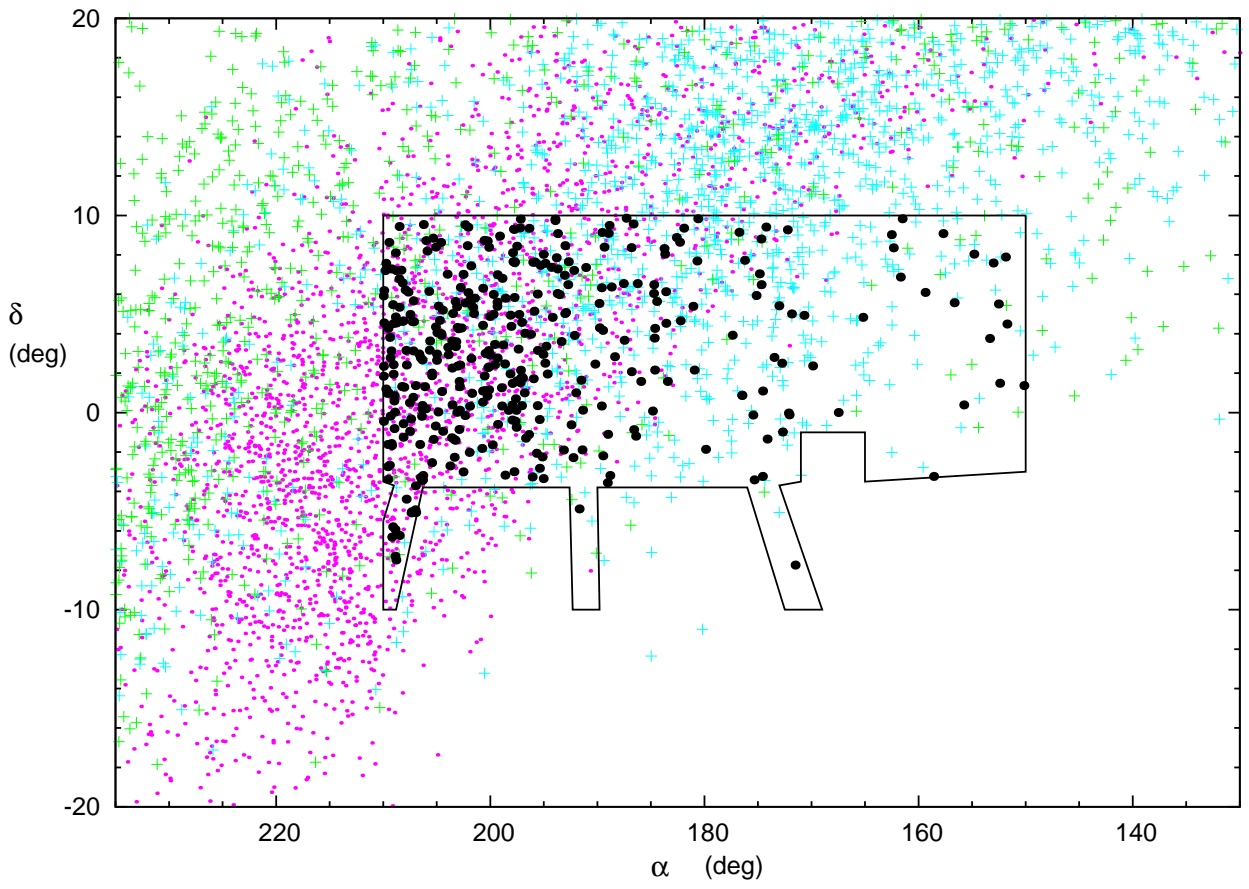

Fig. 15.- Distribution on the sky of the particles (small dots and crosses) in the model of the disruption of the Sgr dSph galaxy by Law \& Majewski (2010b). To preserve clarity, only every other particle is plotted. The dots are particles that were stripped during recent pericenter passages, while the crosses are particles that were stripped during older passages. In the electronic edition of the Journal, the dots are colored magenta and the crosses are colored either cyan or green, following the same color scheme as Law \& Majewski (2010b) for the epoch of stripping. The footprint of the LSQ survey is shown by the polygon. RRLS in the range $40 \leq d_{\odot} \leq 60 \mathrm{kpc}$ are shown as black solid circles. Note that they are concentrated where the younger stream particles (small dots) cross the footprint. See the electronic edition of the Journal for a color version of this figure. 

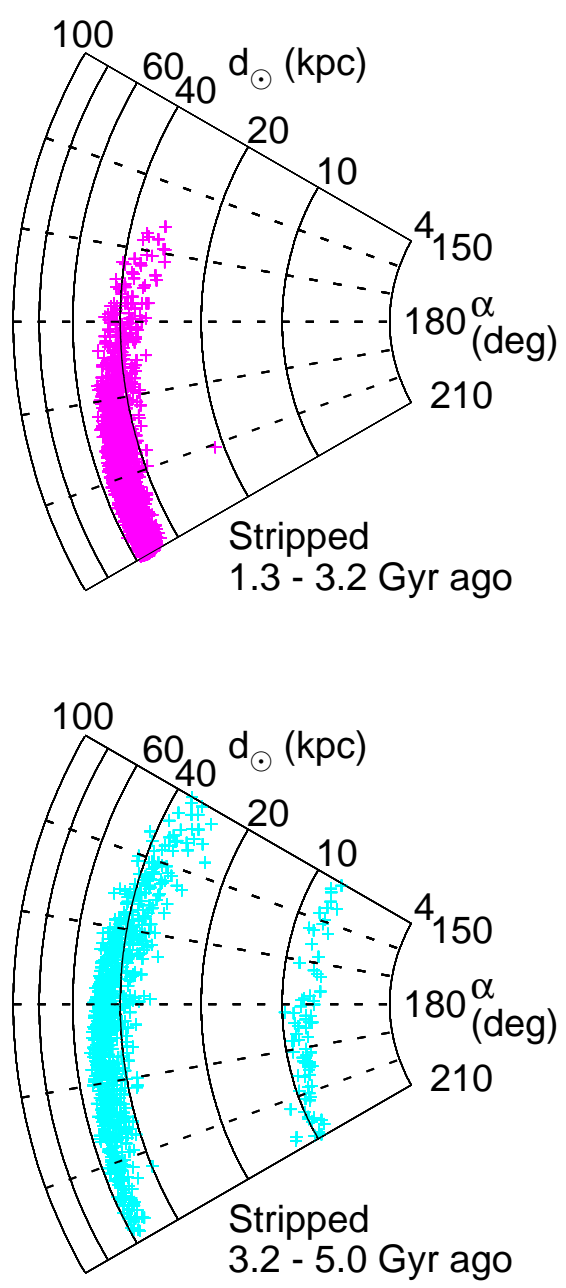

Fig. 16. - The distribution in $\alpha$ and $d_{\odot}$ of the particles in the model that Law \& Majewski (2010b) calculated for the stellar streams from the Sgr dSph galaxy. The top and bottom diagrams show, respectively, the model stars that were separated from the main body of the galaxy $1.3-3.2$ and 3.2-5.0 Gyrs ago. See the electronic edition of the Journal for a color version of this figure, where the color scheme is the same as in Figure [15] and in Law \& Majewski (2010b). 


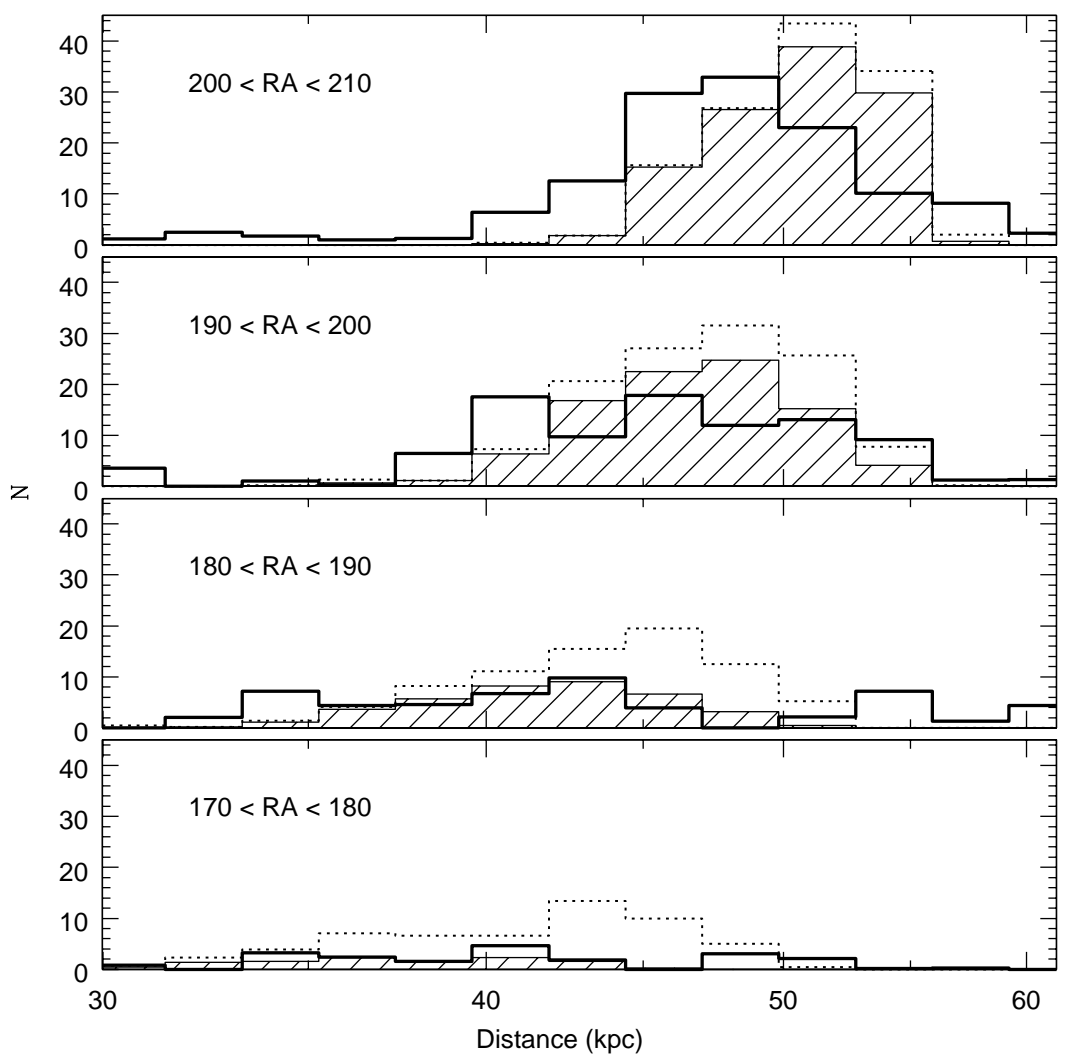

Fig. 17.- Histograms of the distribution of distances of observed RRLS and of particles in the Law \& Majewski (2010b) model of Sgr debris (normalized, see text) in 4 ranges of right ascension. In each panel, the solid line is the number of observed RRLS, the shaded histogram is the number of young stream particles, and the dotted histrogram is the sum of both young and old stream particles. In all 4 panels, the young debris particles appear to match the observed RRLS, although with an offset of about one bin width to larger distances, which is most evident in the upper panels. Note the poor agreement in the lower two panels between the RRLS (solid line) and the sum of the young and old debris particles (dotted line), which suggests that the contribution from the old stream has been overestimated. 


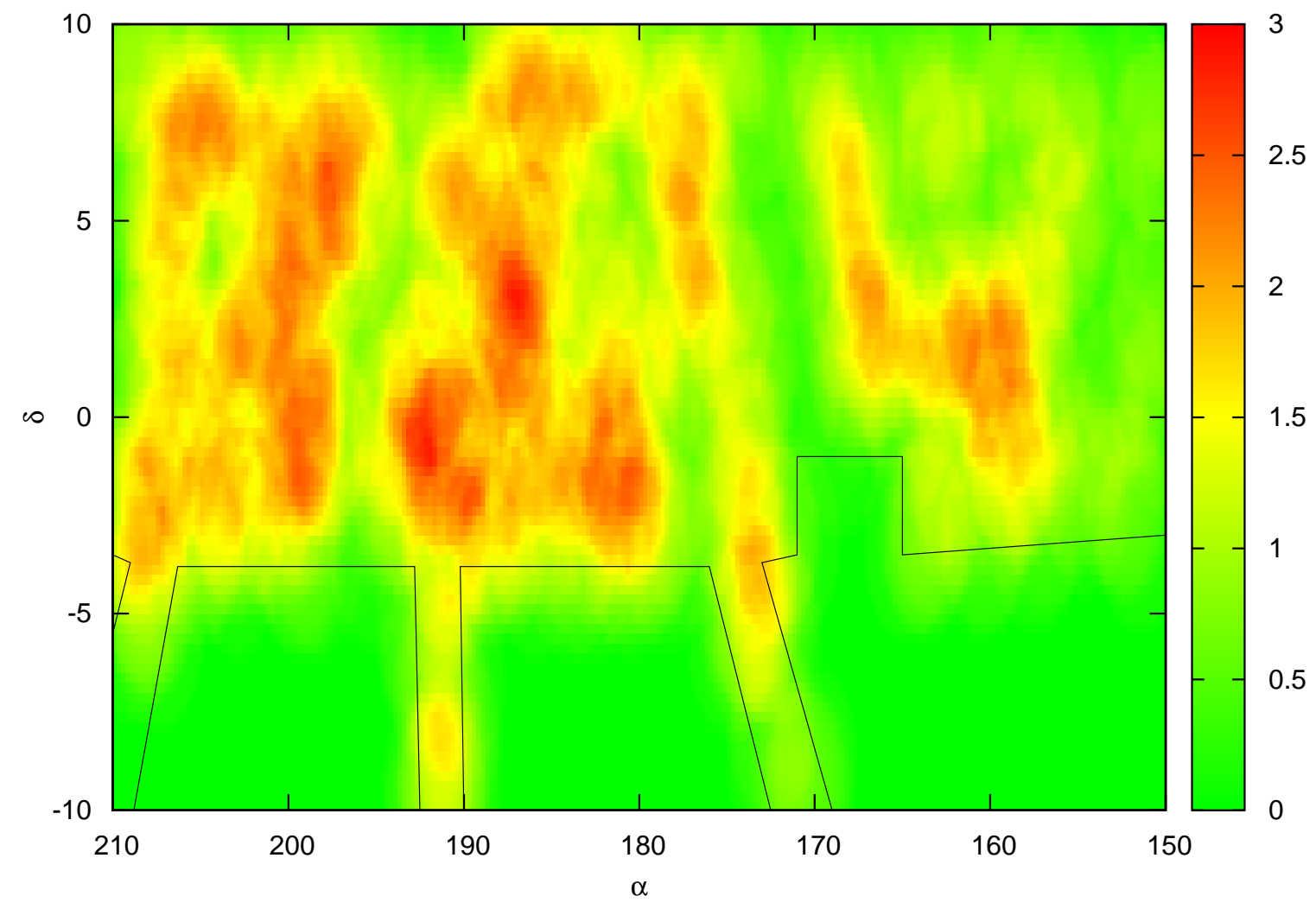

Fig. 18. - The distribution on the sky of the density of RRLS in the range $17 \leq d_{\odot} \leq 22$ kpc. The density scale is the number of standard deviations from the density profile of the smooth halo. The solid line marks the boundary of the LSQ survey. See the electronic edition of the Journal for a color version of this figure. 


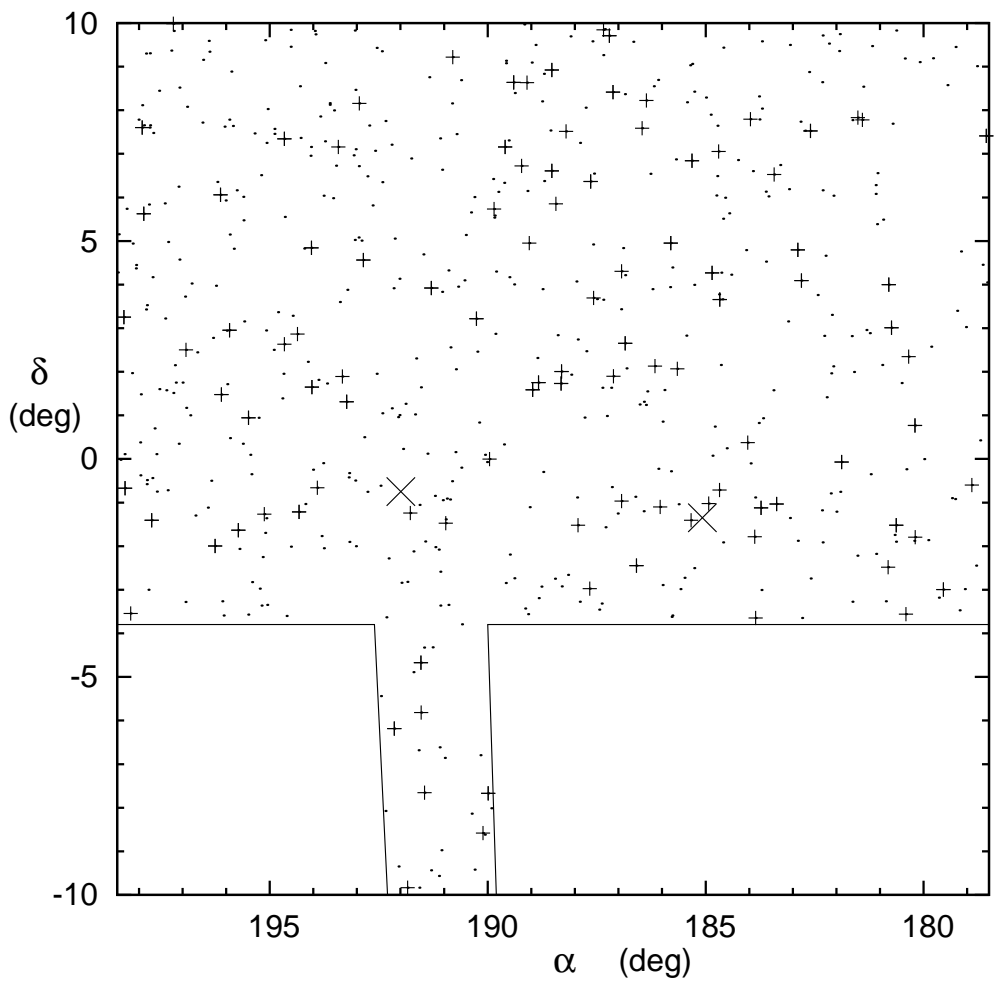

Fig. 19.- The RRLS near the halo substructure identified by Jerjen et al. (2013). The large x's mark the positions of 1220-1 (185.077, -01.35) and 1247-00 (191.992, -00.75). The crosses are LSQ RRLS with $19.5 \leq d_{\odot} \leq 27.5 \mathrm{kpc}$, which may coincide in distance with the substructure. The small dots are LSQ RRLS that lie outside this distance interval. 

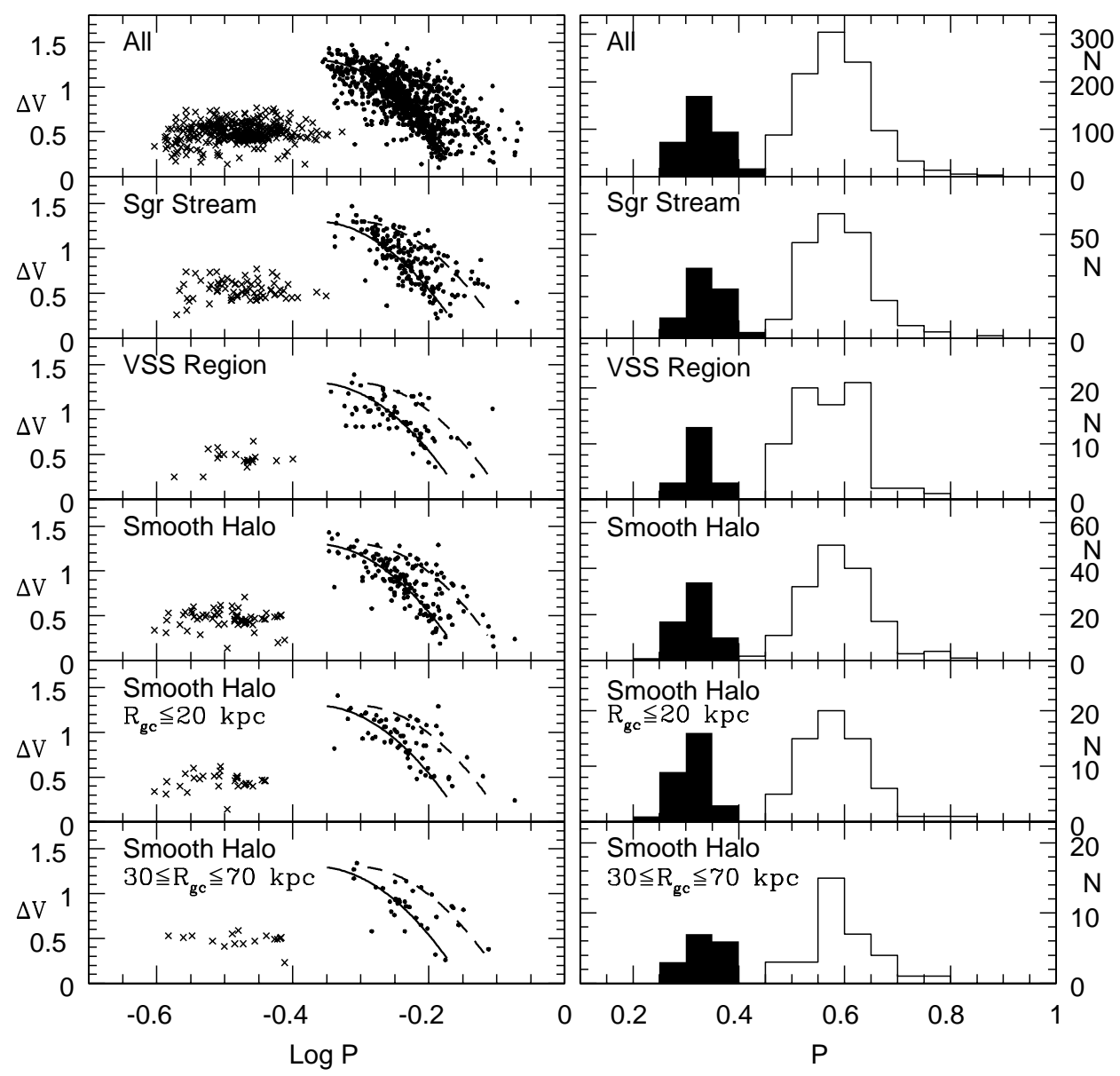

Fig. 20.- The P-A diagrams (left) and the period histograms (right) for the whole sample of LSQ RRLS (minus the ones in the Sex dSph galaxy), for the Sgr stream, and for other subdivisions of the sample (see text). The mean lines for the type ab variables in OoI and OoII globular clusters (Cacciari et al. 2005) are plotted in the P-A diagrams as a solid and dashed curves, respectively. The type c and type ab variables are plotted as x's and dots in the P-A diagrams and as filled and unfilled histograms, respectively. 


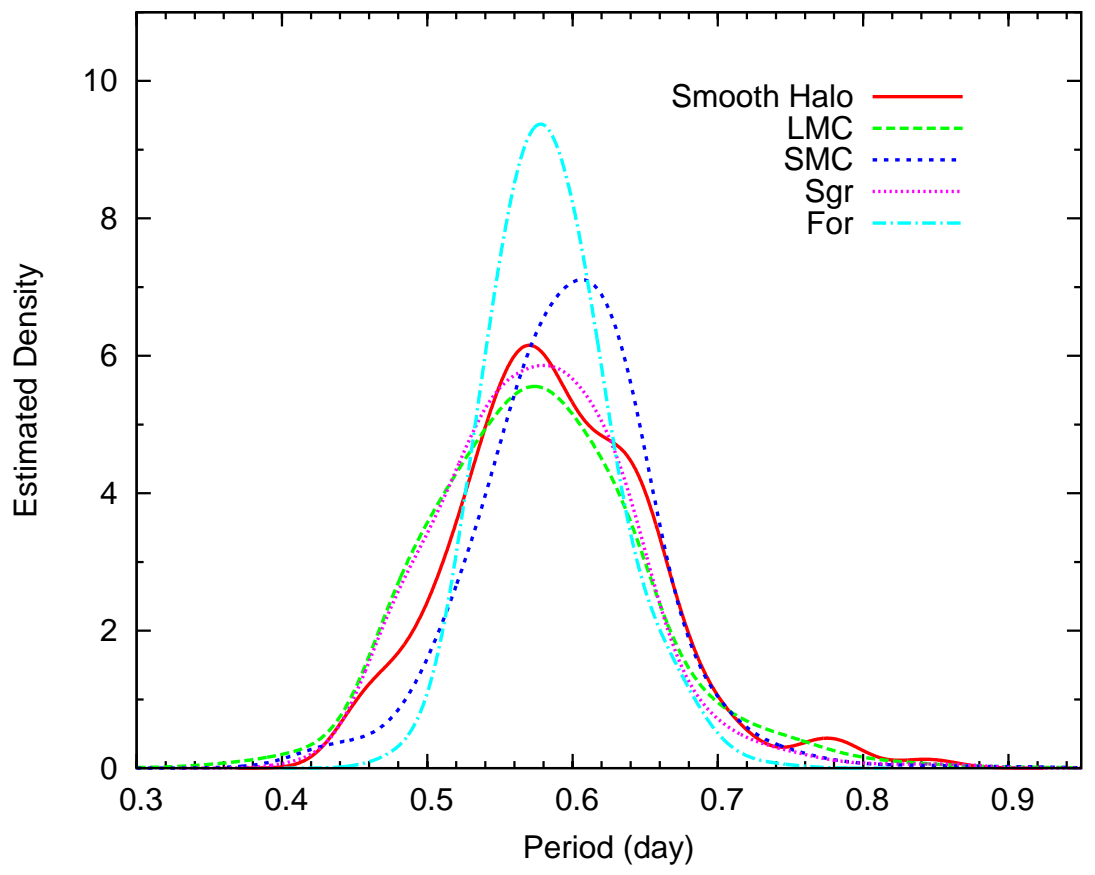

Fig. 21. - The estimated probability density functions for the periods of the type ab variables in the Smooth Halo, LMC, SMC, Sagittarius (main-body), and Fornax are compared. These functions were calculated using a Gaussian kernal with a standard deviation of 0.02 . See the electronic edition of the Journal for a color version of this figure. 


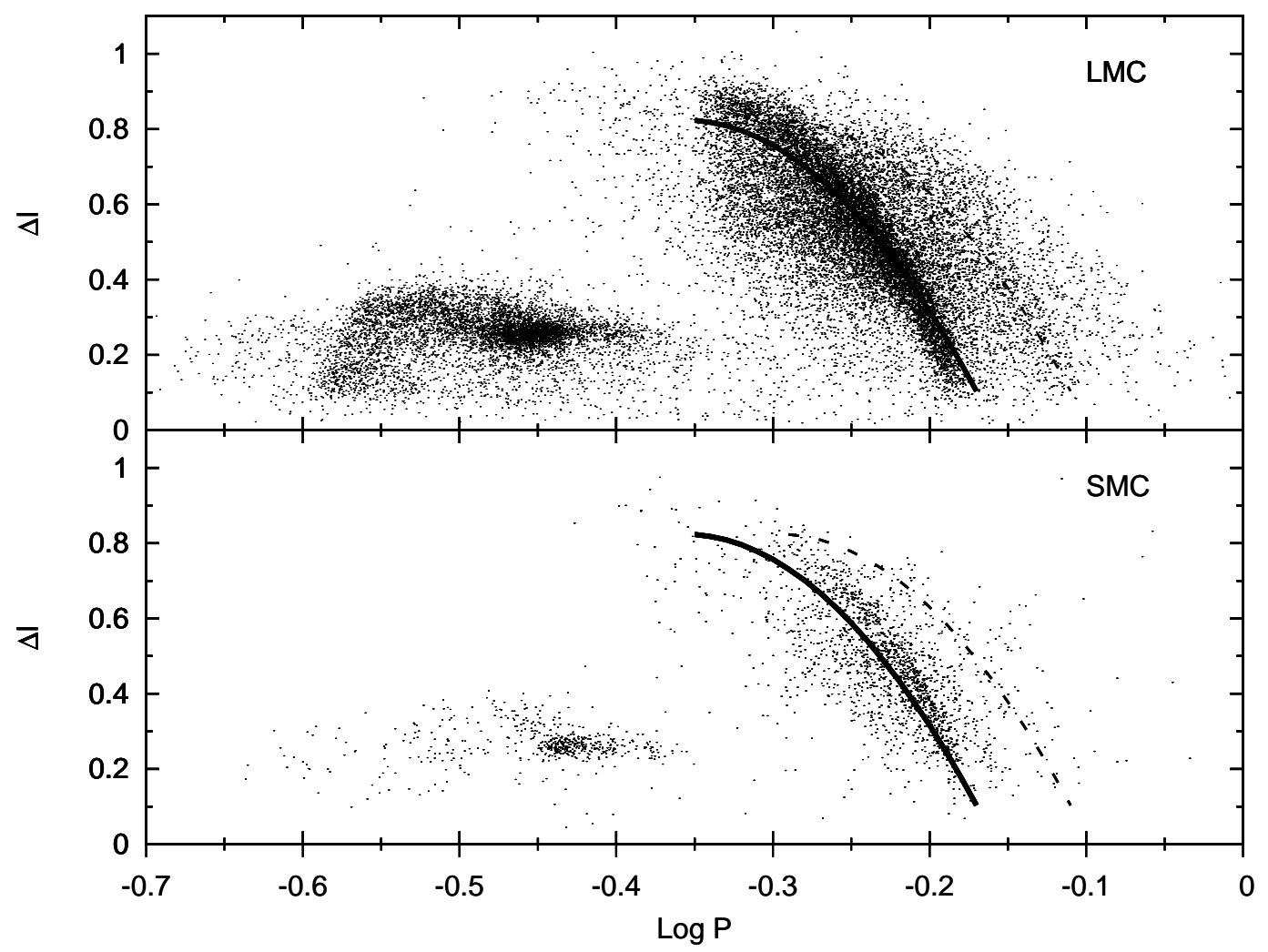

Fig. 22.- The Period-Amplitude diagrams for the RRLS observed by the OGLE project in the LMC and the SMC. The I-band amplitudes, $\Delta I$, of the OoI and OoII curves, solid and dashed lines respectively, were transformed from the V-band to the I-band (see text). 


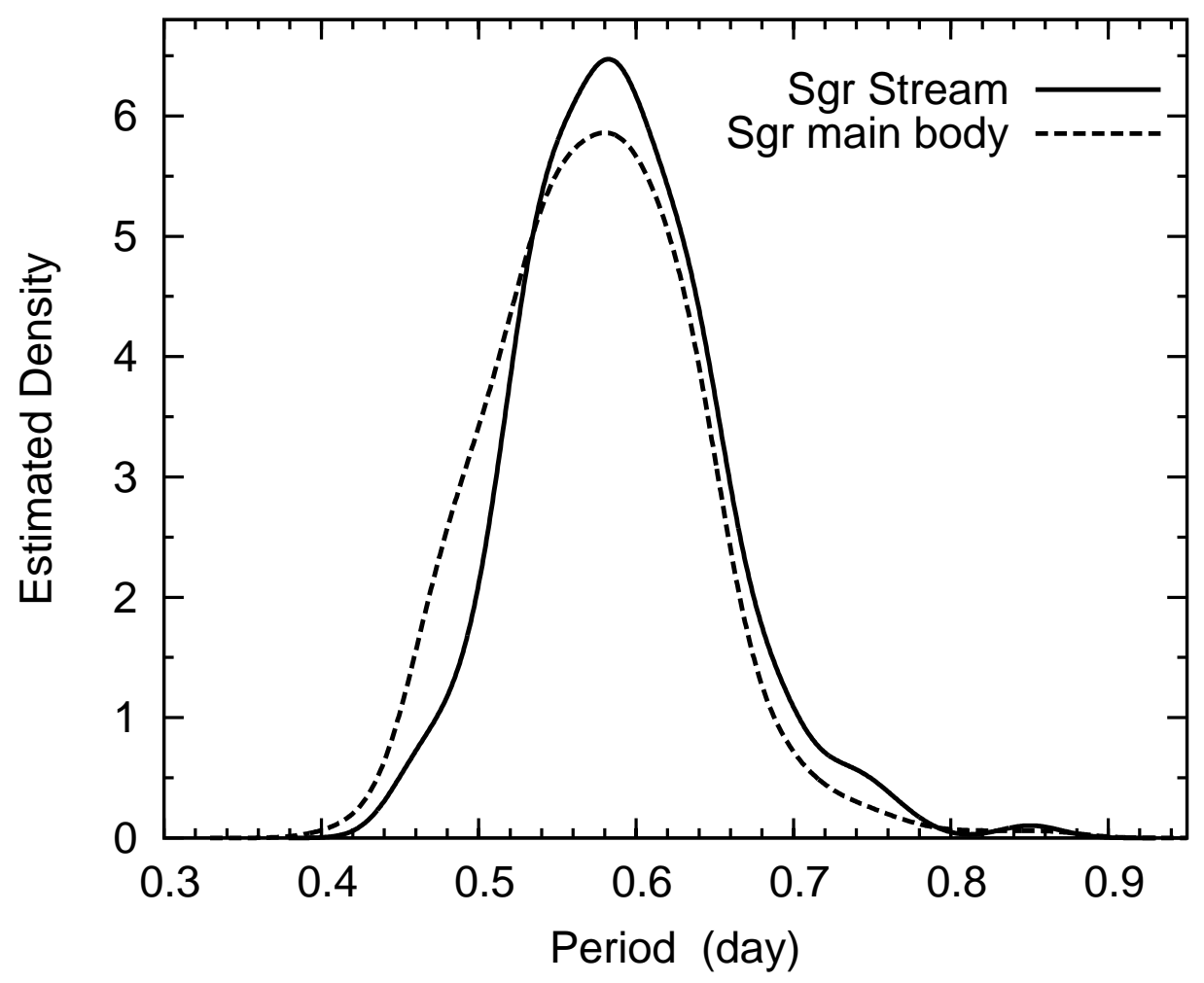

Fig. 23.- The solid and dashed lines are, respectively, the estimated probability density functions for the periods of the type ab RRLS in the Sgr dSph galaxy and for the LSQ RRLS in the Sgr Stream, which were calculated using Gaussian kernal with a standard deviation of 0.02 . 
Table 1. RR Lyrae Stars

\begin{tabular}{|c|c|c|c|c|c|c|c|c|c|c|}
\hline LSQ & $\begin{array}{l}\alpha_{2000} \\
(\mathrm{deg})\end{array}$ & $\begin{array}{l}\delta_{2000} \\
(\operatorname{deg})\end{array}$ & $N_{o b s}{ }^{\mathrm{a}}$ & $\begin{array}{c}\langle V\rangle \\
(\mathrm{mag})\end{array}$ & $\begin{array}{l}\text { Amp } \\
\text { (mag) }\end{array}$ & $\begin{array}{c}\mathrm{P} \\
\text { (day) }\end{array}$ & $\begin{array}{l}\text { HJD }^{b} \\
\text { (day) }\end{array}$ & Type & Flag & Note $^{\mathrm{d}}$ \\
\hline 1 & 150.1707 & 1.3682 & 46 & 18.79 & 1.17 & 0.48960 & 55264.51565 & $a b$ & 0 & $\cdots$ \\
\hline 2 & 150.3709 & 0.1833 & 55 & 16.47 & 1.14 & 0.60535 & 55266.57127 & $a b$ & 0 & $\cdots$ \\
\hline 3 & 150.3886 & 1.7246 & 51 & 15.07 & 1.06 & 0.54243 & 55277.56310 & $a b$ & 0 & $\cdots$ \\
\hline 4 & 150.4103 & 0.2355 & 55 & 18.58 & 0.44 & 0.33643 & 55279.60525 & $\mathrm{c}$ & 0 & $\cdots$ \\
\hline 5 & 150.4330 & 8.6984 & 40 & 17.03 & 0.50 & 0.29651 & 55273.52587 & $\mathrm{c}$ & 0 & $\ldots$ \\
\hline
\end{tabular}

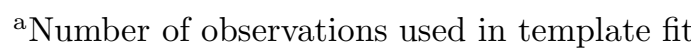

${ }^{\mathrm{b}}$ Heliocentric Julian Date of Maximum Brightness

${ }^{\mathrm{c}}$ Calibration: $0=$ good, $1=$ poor

dSuspected member of the Sex dSph galaxy = Sex dSph; if available V number from Mateo et al. (1995)

Note. - Table 1 is published in its entirety in the electronic edition of The Astrophysical Journal. A portion is shown here for guidance regarding its form and content. 
Table 2. Anomalous Cepheids in the Sextans dSph galaxy

\begin{tabular}{|c|c|c|c|c|c|c|c|c|c|}
\hline LSQ & $\begin{array}{l}\alpha_{2000} \\
(\mathrm{deg})\end{array}$ & $\begin{array}{l}\delta_{2000} \\
(\mathrm{deg})\end{array}$ & $N_{o b s}$ & $\begin{array}{c}\langle V\rangle \\
(\mathrm{mag})\end{array}$ & $\begin{array}{l}\text { Amp } \\
\text { (mag) }\end{array}$ & $\begin{array}{c}\mathrm{P} \\
\text { (day) }\end{array}$ & $\begin{array}{l}\text { HJD }^{\mathrm{a}} \\
\text { (day) }\end{array}$ & $\begin{array}{c}M_{V} \\
(\mathrm{mag})\end{array}$ & Note \\
\hline 24 & 152.9940 & -2.0379 & 47 & 19.98 & 0.48 & 0.45917 & 55280.54211 & +0.22 & $\ldots$ \\
\hline 31 & 153.2278 & -1.5302 & 53 & 19.37 & 0.43 & 0.49278 & 55274.54471 & -0.39 & V19 \\
\hline 34 & 153.3578 & -1.6825 & 44 & 19.89 & 0.30 & 0.50640 & 55273.63399 & +0.13 & V34 \\
\hline 36 & 153.4017 & -1.6314 & 51 & 18.88 & 0.89 & 0.69300 & 55286.51409 & -0.88 & V1 \\
\hline 40 & 153.6185 & -1.6865 & 50 & 18.64 & 0.60 & 2.02186 & 55281.52954 & -1.12 & Q119 \\
\hline 55 & 154.9881 & -2.0505 & 46 & 19.55 & 0.88 & 0.42806 & 55278.55108 & -0.21 & $\ldots$ \\
\hline
\end{tabular}

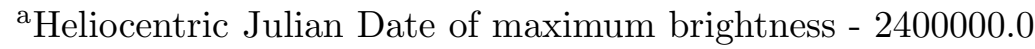

Note. - V and Q numbers from Mateo et al. (1995) and Vivas et al. (2004), respectively. 
Table 3. The Observations

\begin{tabular}{cccc}
\hline \hline LSQ & HJD & V & std. dev. \\
& $($ day $)$ & $(\mathrm{mag})$ & $(\mathrm{mag})$ \\
\hline & & & \\
1 & 55286.515908 & 17.960 & 0.024 \\
1 & 55261.565315 & 17.920 & 0.011 \\
1 & 55260.600868 & 17.958 & 0.013 \\
1 & 55262.587966 & 18.167 & 0.012 \\
1 & 55286.558406 & 18.294 & 0.032 \\
\hline
\end{tabular}

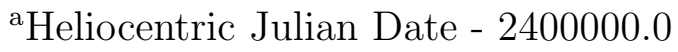

${ }^{\mathrm{b}}$ Based on photon statistics

Note. - Table 3 is published in its entirety in the electronic edition of The Astrophysical Journal. A portion is shown here for guidance regarding its form and content. 
Table 4. RR Lyrae Variables near 1220-1

\begin{tabular}{cccccccc}
\hline \hline LSQ & $\alpha_{2000}$ & $\delta_{2000}$ & Type & $\langle V\rangle$ & $\langle V\rangle_{0}$ & $d_{\odot}$ & $\phi^{\mathrm{a}}$ \\
& $(\mathrm{deg})$ & $(\mathrm{deg})$ & & $(\mathrm{mag})$ & $(\mathrm{mag})$ & $(\mathrm{kpc})$ & $(\mathrm{deg})$ \\
\hline & & & & & & & \\
515 & 184.67940 & -0.71070 & $\mathrm{c}$ & 17.18 & 17.07 & 20.2 & 0.754 \\
525 & 184.92920 & -1.02490 & $\mathrm{c}$ & 17.73 & 17.63 & 26.0 & 0.358 \\
532 & 185.33610 & -1.40380 & $\mathrm{ab}$ & 17.77 & 17.66 & 26.4 & 0.262 \\
550 & 186.04470 & -1.09790 & $\mathrm{ab}$ & 17.77 & 17.69 & 26.8 & 0.997 \\
\hline
\end{tabular}

aAngular distance from the center of 1220-1 
Table 5. Oosterhoff characteristics of the LSQ survey and massive MW satellites

\begin{tabular}{|c|c|c|c|c|c|c|c|c|}
\hline Sample & $\begin{array}{l}\left\langle P_{a b}\right\rangle \\
(\text { day })\end{array}$ & $\begin{array}{l}\sigma P_{a b} \\
(\text { day })\end{array}$ & $\begin{array}{c}\left\langle P_{c}\right\rangle \\
\text { (day) }\end{array}$ & $\begin{array}{c}\sigma P_{c} \\
\text { (day) }\end{array}$ & $n_{a b}$ & $n_{c}$ & $n_{c} /\left(n_{a b}+n_{c}\right)$ & OoII \% \\
\hline All LSQ & 0.586 & 0.068 & 0.333 & 0.040 & 1007 & 358 & $0.26 \pm 0.02$ & $27 \pm 2$ \\
\hline Sgr Stream & 0.591 & 0.062 & 0.337 & 0.038 & 194 & 70 & $0.27 \pm 0.04$ & $32 \pm 5$ \\
\hline VSS Region & 0.574 & 0.063 & 0.336 & 0.035 & 73 & 20 & $0.22 \pm 0.05$ & $24 \pm 7$ \\
\hline Smooth Halo & 0.589 & 0.069 & 0.321 & 0.034 & 160 & 62 & $0.28 \pm 0.04$ & $25 \pm 5$ \\
\hline \multicolumn{9}{|l|}{ Smooth Halo } \\
\hline$R_{g c}<20 \mathrm{kpc}$ & 0.585 & 0.069 & 0.312 & 0.033 & 64 & 28 & $0.30 \pm 0.07$ & $29 \pm 8$ \\
\hline \multicolumn{9}{|l|}{ Smooth Halo } \\
\hline $30<R_{g c}<70 \mathrm{kpc}$ & 0.596 & 0.065 & 0.336 & 0.041 & 34 & 16 & $0.32 \pm 0.10$ & $33 \pm 12$ \\
\hline Sgr dSph & 0.575 & 0.064 & 0.326 & 0.034 & 1241 & 287 & $0.19 \pm 0.01$ & $\ldots$ \\
\hline For dSph & 0.585 & 0.040 & 0.359 & 0.056 & 397 & 118 & $0.23 \pm 0.02$ & $\cdots$ \\
\hline $\mathrm{LMC}$ & 0.576 & 0.073 & 0.329 & 0.041 & 17693 & 7213 & $0.29 \pm 0.00$ & $\cdots$ \\
\hline $\mathrm{SMC}$ & 0.596 & 0.059 & 0.358 & 0.040 & 1933 & 542 & $0.22 \pm 0.01$ & $\ldots$ \\
\hline OoI GCs & 0.55 & $\cdots$ & 0.32 & $\cdots$ & $\cdots$ & $\cdots$ & 0.17 & $\ldots$ \\
\hline OoII GCs & 0.64 & $\ldots$ & 0.37 & $\ldots$ & $\ldots$ & $\ldots$ & 0.44 & . \\
\hline
\end{tabular}

